\title{
Air Quality Modelling for Informing Air Pollution Policy
}

\author{
by
}

Amanda Joy Pappin

A thesis submitted to the Faculty of Graduate and Postdoctoral Affairs in partial fulfillment of the requirements for the degree of

Doctor of Philosophy

in

Environmental Engineering

Carleton University

Ottawa, Ontario

(C) 2016, Amanda Joy Pappin 


\section{Abstract}

Managing air quality through emissions control entails significant societal benefits in Canada and around the world. As the public health impacts of emissions depend on the atmospheric conditions conducive to pollutant transport and transformation, sophisticated atmospheric models are necessary to link public health impacts with sources of emissions directly. This thesis develops a novel method to integrate health benefit assessment and formal sensitivity analysis tools. It employs a reverse sensitivity analysis technique, infused with epidemiological and economic data, to attribute air pollution health effects to emissions sources. This linkage creates a streamlined approach for assessing the damages incurred by anthropogenic emissions, and the benefits of their control, on a source-by-source basis.

The findings presented in this thesis indicate that the public health benefits of emission controls vary considerably from source-to-source and by emitted species. A main feature of emission control benefits is their dependency on the composition of the atmosphere and hence on emission quantities. As the atmosphere becomes cleaner with progressive emission reduction policies, the benefits-per-ton of emissions control are likely to change, particularly for pollutants that undergo nonlinear transformations in the atmosphere. Further, the shape of the concentration-response function (CRF) between pollutant exposure and mortality plays a determining role in estimating these benefits. This thesis investigates how both atmospheric chemistry and assumptions about the CRF influence the health benefits of emission control. For secondary pollutants such as ozone, the benefits-per-ton of $\mathrm{NO}_{\mathrm{x}}$ control are found to increase substantially as the atmosphere 
becomes less polluted. For primary pollutants such as $\mathrm{NO}_{2}$, compounding benefits of $\mathrm{NO}_{x}$ control exist due entirely to a supralinear CRF.

The findings of this thesis indicate unforeseen and long-term benefits of emissions control, suggesting that current emission controls make future abatement efforts more worthwhile. Assessment of recent emission trends in North America indicates that we are currently at an important point on the abatement trajectory, where the benefits of emission controls have increased in the past and will do so considerably in the near future. Regard for the compounding nature of emission control benefits can cast abatement policies in a self-propagating, self-rewarding light in the long-term. 


\section{Preface}

This thesis consists of copyrighted and non-copyrighted manuscripts. The following published manuscripts have been reproduced in Chapters 4-7 of this thesis with permission of the publishers and co-authors:

Pappin A, Hakami A. 2013. Source attribution of health benefits from air pollution abatement in Canada and the United States: An adjoint sensitivity analysis. Environ Health Perspect 121:572-579.

Pappin AJ, Hakami A. 2013. Attainment vs exposure: Ozone metric responses to source-specific $\mathrm{NO}_{\mathrm{x}}$ controls using adjoint sensitivity analysis. Environ Sci Technol 47:13519-13527.

Pappin AJ, Mesbah SM, Hakami A, Schott S. 2015. Diminishing returns or compounding benefits of air pollution control? The case of $\mathrm{NO}_{\mathrm{x}}$ and ozone. Environ Sci Technol 49:9548-9556.

Pappin AJ, Hakami A, Blagden P, Nasari M, Szyszkowicz M, Burnett RT. 2016. Health benefits of reducing $\mathrm{NO}_{\mathrm{x}}$ emissions in the presence of epidemiological and atmospheric nonlinearities. Environ Res Lett 11:064015. 
A draft manuscript to be submitted to a scientific journal is presented in Chapter 8 of this thesis with permission of the co-authors:

\author{
Pappin AJ, Hakami A, Bell ML. $\mathrm{NO}_{\mathrm{x}}$ control: A gift that keeps on giving. In \\ preparation.
}

Amanda Pappin declares that she is the author of the entire thesis and has played the main or sole role in conducting the research described in Chapters 4-8. She was the main contributor and was responsible for designing the methods, conducting original research, preparing and analyzing data, and interpreting the findings in this thesis. Statements of the percentage contribution made by Amanda Pappin and the copyright information for each of the manuscripts are provided at the beginning of the corresponding chapter. Chapter 6 contains a published paper led collaboratively by both Amanda Pappin and Dr. Morteza Mesbah. Dr. Morteza Mesbah conducted an initial investigation on the research topic and Amanda Pappin updated and expanded this analysis using improved methodology and an updated set of model data, ran additional analysis to further demonstrate the findings, and prepared the final manuscript included in Chapter 6. Chapter 7 was a collaborative work that involved Dr. Rick Burnett, Phil Blagden, Dr. Masoud Nasari, and Dr. Mieczyslaw Szyszkowicz at Health Canada. The team of researchers at Health Canada provided epidemiological modelling data in support of the air quality modelling conducted by Amanda Pappin in Chapter 7.

Dr. Paul Van Geel, in his capacity as Amanda Pappin's supervisor, confirms that the assessment made by the candidate above is a valid reflection of her contributions. Dr. Van Geel assumed the role of Amanda Pappin's thesis supervisor in the final year of her 
$\mathrm{PhD}$ studies. At that time, the papers contained in Chapters 4 and 5 were published, the paper which forms Chapter 6 was under final review with the publishing journal, and the papers which form Chapters 7 and 8 had been prepared in full. Dr. Van Geel oversaw the assembly of the thesis and reviewed the thesis but did not contribute to the technical content of the thesis or the final papers and as such does not appear as a co-author. Dr. Amir Hakami was Amanda Pappin's initial PhD supervisor and was her supervisor for a majority of her PhD studies. As such he, and members of his research team and collaborators, appear as co-authors of the papers. 


\section{Acknowledgements}

These brief lines cannot begin to reflect my level of appreciation for those who have guided me during my $\mathrm{PhD}$.

I would like to thank Dr. Amir Hakami for teaching me much of what I know. Thank you for your vision, your wisdom, and your friendship. I am forever changed because of it.

I would like to thank Dr. Paul Van Geel for supervising me in the last year of my degree. I would also like to express my appreciation to the members of my thesis examination committee, Dr. Jeff Brook, Dr. Paul Villeneuve, Dr. Handan Tezel, and Dr. Deniz Karman, for their advice, their questioning, and for testing the depths of my knowledge.

I would like to thank Dr. Michelle Bell and her research group at Yale University for hosting me. Thank you for your wise advice and for offering me a new perspective that has helped shape my career.

I extend my thanks to Dr. Rick Burnett and Phil Blagden at Health Canada for the insightful discussions that inspired and guided the later chapters of my thesis. Thank you to Stan Judek as well, without whom this thesis would not have evolved the way it did.

An immense thanks to Dr. Shunliu Zhao at Carleton University for his work on adjoint development. Thank you to Dr. Morteza Mesbah for his patience and for transferring so much of his knowledge to me in the early years of my PhD. Many thanks 
to Matthew Russell, who never hesitated to lend a helping hand. Thank you to others in the office, namely Nicole MacDonald, Saba Hajaghassi, Sahar Saeednooran, Pedram Falsalfi, Melanie Fillingham, Robyn Chatwin-Davies, Dr. Rabab Mashayekhi, Angele Genereux, Sina Voshtani, Shabnam Mizani, Natalie Linklater, and Mandy Witteman, for their never-ending openness and support, and for always keeping things interesting.

Lastly, I would like to thank my family, who has stayed by my side through all the twists and turns of my degree. Thank you for your unconditional love and support.

Funding for the research presented in this thesis was provided by the Natural Sciences and Engineering Research Council of Canada (NSERC), Health Canada, and Fulbright Canada. 


\section{Table of Contents}

1 Chapter: Introduction ......................................................................................................... 1

2 Chapter: Background and Literature Review ........................................................6 6

2.1 Epidemiologic Evidence Linking Air Pollution Exposure and Mortality ............. 6

2.1.1 Epidemiologic Studies on Ozone …………….......................................... 8

2.1.2 Epidemiologic Studies on Nitrogen Dioxide ................................................ 11

2.1.3 Epidemiologic Studies on Particulate Matter ............................................... 15

2.2 Epidemiologic and Valuation Data in Health Impact Assessment ...................... 18

2.2.1 Air Quality Health Impact Assessment Studies .......................................... 19

2.3 Health Impacts of Emission Reductions ........................................................... 21

3 Chapter: Methods ................................................................................................................. 27

3.1 Atmospheric Chemical Transport Models …………......................................... 27

3.1.1 Community Multiscale Air Quality Model (CMAQ) ....................................29

3.2 Sensitivity Analysis Methods ........................................................................... 30

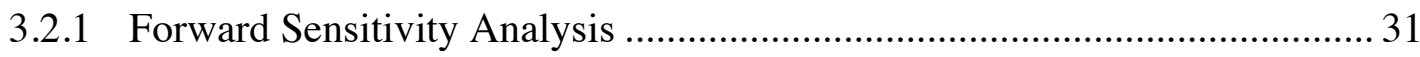

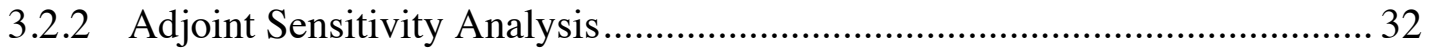

3.3 Estimating Air Pollution Health Impacts ................................................................... 35

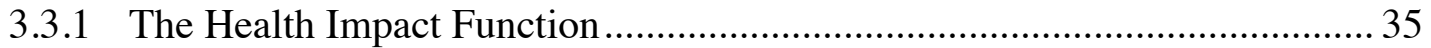

3.3.2 Quantifying Health Impacts Using Adjoint Sensitivity Analysis ................ 37

3.3.3 Distinguishing Between Short- and Long-term Health Impacts in Adjoint Sensitivity Analysis ……………………………………................................. 38

4 Chapter: Source Attribution of Health Benefits .......................................................... 40

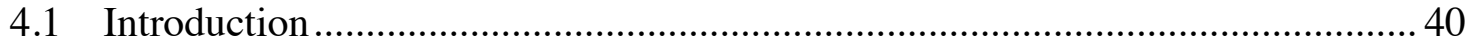

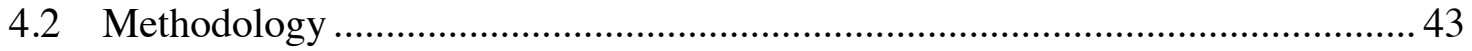

4.2.1 Adjoint Sensitivity Analysis..................................................................... 43

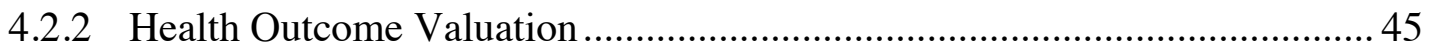

4.2.3 Health Benefit Estimation Case Study ......................................................... 47

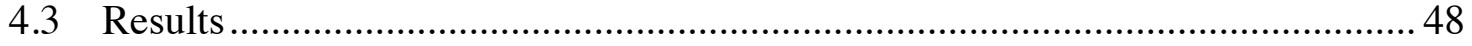

4.3.1 Attribution of Canadian Health Benefits to North American Sources .......... 50

4.3.2 Attribution of U.S. Health Benefits to North American Sources ................... 50

4.3.3 Temporal Variability in Health Benefit Influences ......................................... 52 


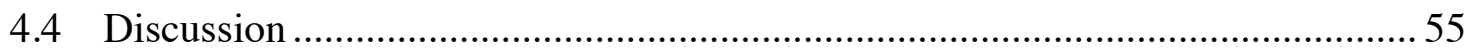

4.4.1 Cross-border Transport of Health Benefits ……………………………..... 55

4.4.2 Effect of Averaging Period on Health Benefit Influences ………………..... 56

4.4.3 Health Benefit Influences of Unit Source Reductions ....................................58

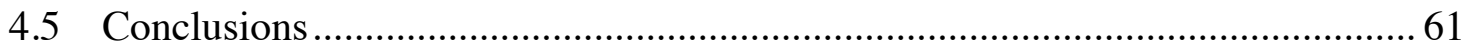

5 Chapter: Policy Analysis of Emission Control Benefits based on Attainment and Exposure Metrics ......................................................................................................................6 64

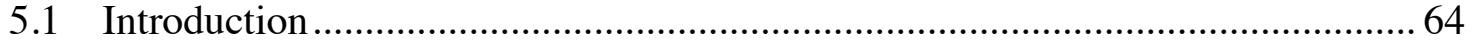

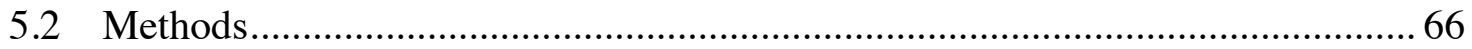

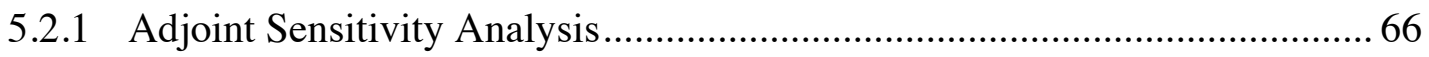

5.2.2 Attainment: Probabilistic Design Value Estimation ...................................... 68

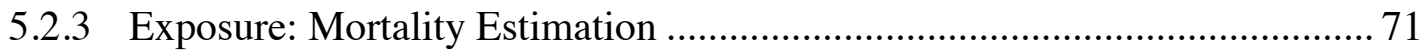

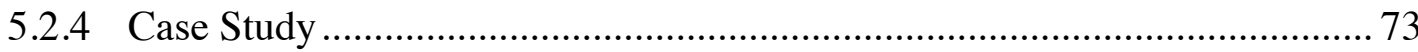

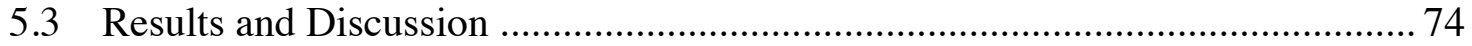

5.3.1 Metric Responses to Location-Specific Source Reductions ......................... 75

5.3.2 Metric Responses to Day-Specific Source Reductions .................................. 78

5.3.3 Variation of Exposure-Based Metric Responses with Averaging Period ..... 80

5.3.4 Policy Considerations of Coupled Attainment and Exposure-Based Metric

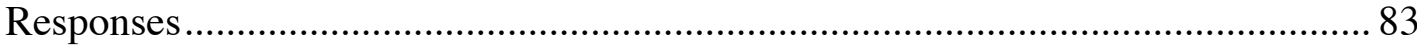

\section{Chapter: Diminishing Returns or Compounding Benefits of Air Pollution}

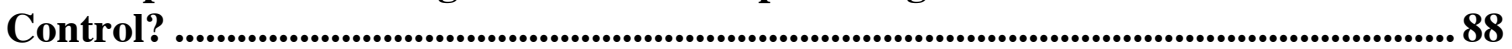

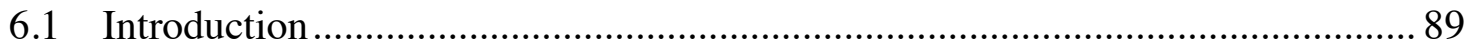

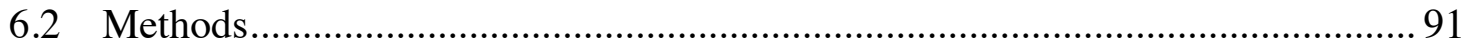

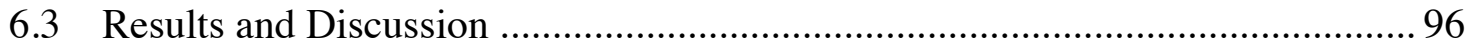

7 Chapter: Compounding Benefits in the Presence of Epidemiological and Atmospheric Nonlinearities........................................................................................................ 112

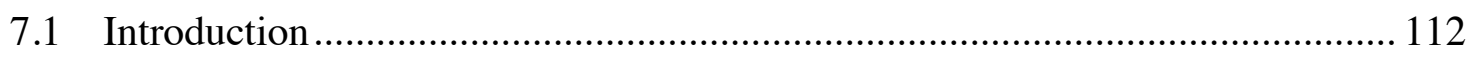

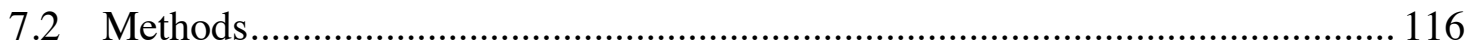

7.2.1 Adjoint Formulation .............................................................................. 117

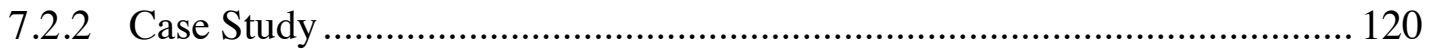

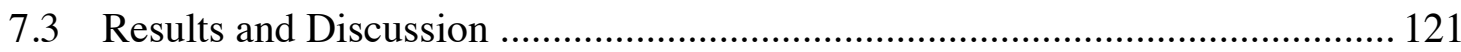

7.3.1 $\mathrm{NO}_{2}$-based Benefits-per-ton Based on Linear in Concentration and Log-

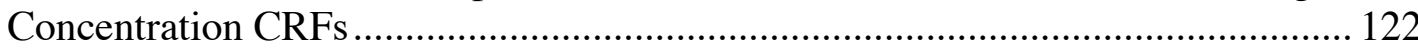

7.3.2 $\mathrm{O}_{3}$-based Benefits-per-ton Based on a Linear CRF.................................... 130 
7.3.3 Other Pollutants ................................................................................ 133

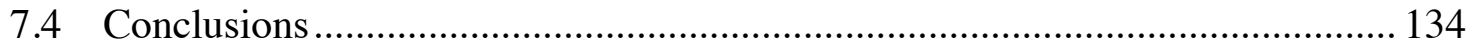

8 Chapter: NO ${ }_{x}$ Control - A Gift that Keeps on Giving............................................ 136

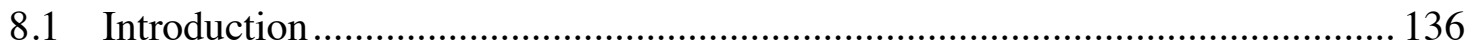

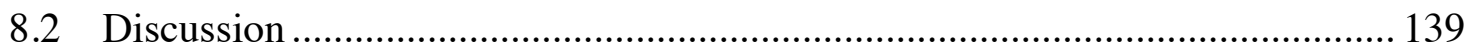

8.2.1 Marginal Benefits in the Face of Emission Reductions ........................... 141

8.2.2 Marginal Benefits in the Face of a Warming Climate .............................. 144

8.2.3 The Role of Advancing Epidemiological Evidence in Marginal Benefit

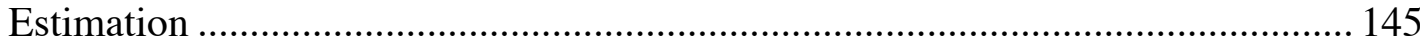

8.2.4 Marginal Costs in the Face of Technological Advancement and Learning by Doing 147

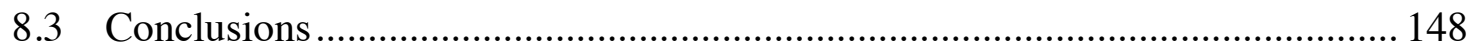

9 Chapter: Conclusion ............................................................................... 150

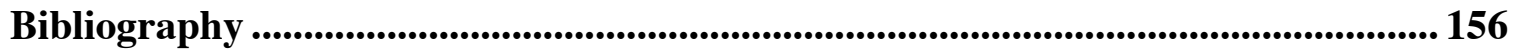




\section{List of Figures}

Figure 1.1 Multi-disciplinary context of sensitivity analysis for decision-making ......... 1

Figure 2.1 CMAQ Input Processors and CCTM .................................................... 30

Figure 3.1 Health benefit influences of a $10 \%$ reduction in emissions of $\mathrm{NO}_{\mathrm{x}}$ and $\mathrm{VOCs}$

Figure 4.2 Daily variability of influences from a 10\% reduction in emissions of $\mathrm{NO}_{\mathrm{x}}$ originating from major cities

Figure 4.3 Influences on U.S. short-term mortality estimated for various averaging periods

Figure 4.4 Influences of 1 tonne reductions in anthropogenic emissions of $\mathrm{NO}_{\mathrm{x}}$ and VOCs

Figure 5.1 Average influences of anthropogenic $\mathrm{NO}_{\mathrm{x}}$ emission reductions on attainment and exposure

Figure 5.2 Daily influences of a $10 \%$ reduction in anthropogenic $\mathrm{NO}_{\mathrm{x}}$ from major cities on nation-wide attainment and exposure 80

Figure 5.3 Differences between influences on exposure-based metrics for various averaging periods 83

Figure 5.4 Effectiveness categories of coupled metric responsiveness to emission controls

Figure 6.1 Marginal benefits for $\mathrm{NO}_{\mathrm{x}}$ emitted from mobile and point sources across the U.S.

Figure 6.2 $\mathrm{NO}_{\mathrm{x}}$ marginal benefits as a function of U.S.-wide abatement level for a sample of source locations

Figure 6.3 Average U.S.-wide mobile- and point-marginal benefit curves for various levels of U.S.-wide abatement of all species

Figure 6.4 Total U.S.-wide damage as a function of U.S.-wide abatement of mobile sources, point sources, and both simultaneously 104

Figure 6.5 Depiction of the economic equilibrium point between marginal benefit and marginal cost.

Figure 7.1 Examples of linear and supralinear forms of CRFs.

Figure 7.2 $\mathrm{NO}_{2}$-based benefits-per-ton for Canada for linear in concentration and logconcentration CRFs at baseline 2007 emission levels

Figure 7.3 Benefits-per-ton of $\mathrm{NO}_{\mathrm{x}}$ control based on a log-concentration $\mathrm{CRF}$ for $\mathrm{NO}_{2}$ at various abatement levels 126

Figure 7.4 Benefits-per-ton for major cities in Canada 127 
Figure 7.5 Benefits-per-ton based on log-concentration vs linear in concentration CRFs for $\mathrm{NO}_{2}$ at various abatement levels ..................................................................... 129

Figure 7.6 Benefits-per-ton of $\mathrm{NO}_{\mathrm{x}}$ control based on a linear in concentration CRF for $\mathrm{O}_{3}$ at various abatement levels.

Figure 8.1 Traditional depiction of marginal benefit and marginal cost as a function of emission level.................................................................................................. 140

Figure 8.2 An alternative shape of the $\mathrm{NO}_{\mathrm{x}}$ marginal benefit curve........................... 143 


\section{List of Tables}

Table 7.1 Risk estimates and CRFs from the CanCHEC study used in estimating

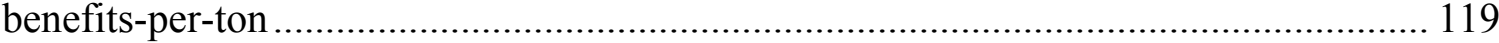




\section{List of Appendices}

Appendix A: The Chemistry of Ground-level Ozone Production .............................. 175

Appendix B: Supplementary Material for Chapter 4 ............................................ 179

Appendix C: Supplementary Material for Chapter 5 .................................................. 183

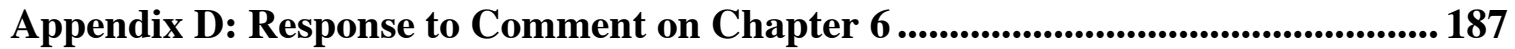




\section{List of Abbreviations}

ACS CPS-II American Cancer Society Cancer Prevention Study II

ADE atmospheric diffusion equation

APHENA Air Pollution and Health: A Combined European and North American

Approach

APHEA-2 Air Pollution and Health: A European Approach

AQBAT Air Quality Benefits Assessment Tool

AQHI Air Quality Health Index

BC black carbon

BCON Boundary Conditions Processor

BenMAP Environmental Benefits Mapping and Analysis Program

BMR baseline mortality rate

BPT benefit-per-ton

CanCHEC Canadian Census Health and Environment Cohort

CCTM CMAQ Chemistry Transport Model

CDC Centers for Disease Control and Prevention

CMAQ Community Multiscale Air Quality model

CPF climate penalty factor

CRF concentration-response function

CTM chemical transport model

DDM decoupled direct method

DM8A daily maximum 8-hour average 


$\begin{array}{ll}\text { EPA } & \text { Environmental Protection Agency } \\ \text { GBD } & \text { Global Burden of Disease } \\ \text { HR } & \text { hazard ratio } \\ \text { ICD } & \text { International Classification of Disease } \\ \text { ICON } & \text { Initial Conditions Processor } \\ \text { ISA } & \text { Integrated Science Assessment } \\ \text { JPROC } & \text { Photolysis Rate Processor } \\ \text { MB } & \text { marginal benefit } \\ \text { MC } & \text { marginal cost } \\ \text { MCIP } & \text { Meteorology Chemistry Interface Processor } \\ \text { MD } & \text { marginal damage } \\ \text { MFE } & \text { mean fractional error } \\ \text { MFB } & \text { mean fractional bias } \\ \text { NEI } & \text { National Emission Inventory } \\ \text { NMMAPS } & \text { National Morbidity, Mortality and Air Pollution Study } \\ \text { NPRI } & \text { National Pollutant Release Inventory } \\ \text { OC } & \text { organic carbon } \\ \text { PAH } & \text { polycyclic aromatic hydrocarbon } \\ \text { PDV } & \text { probabilistic design value } \\ \text { PM } & \text { particulate matter } \\ \text { RR } & \text { particulate matter with aerodynamic diameter }<2.5 \mu m \\ \text { relative risk } \\ \text { Sparse Matrix Operator Kernel Emissions model } \\ \text { M }\end{array}$




$\begin{array}{ll}\text { TLM } & \text { tangent linear model } \\ \text { VOC } & \text { volatile organic compound } \\ \text { VSL } & \text { value of statistical life } \\ \text { WHO } & \text { World Health Organization } \\ \text { WRF } & \text { Weather Research and Forecasting model }\end{array}$




\section{List of Symbols}

Note that this integrated thesis is a collection of published and unpublished papers in which the symbols used may differ for certain variables.

$\alpha$

domain of model inputs

$\beta$

risk or effect estimate

$\varphi \quad$ adjoint forcing term

$\phi \quad$ adjoint forcing term

$\rho \quad$ air density

$\lambda \quad$ adjoint variable

$\omega \quad$ grid cell location

$B \quad$ monetized health benefit

C pollutant concentration

$e \quad$ emission rate

$E \quad$ emission rate

$F \quad$ chemistry operator

$J \quad$ adjoint cost function

$K \quad$ diffusivity coefficient

L linear model operator

M nonlinear model operator, mortality

$\mathrm{M}_{0} \quad$ baseline mortality rate

$n \quad$ model simulation days 


$\begin{array}{ll}p & \text { probability } \\ P & \text { population } \\ R & \text { chemical reaction rate } \\ u & \text { wind velocity } \\ V A L & \text { marginal valuation function } \\ V_{S L} & \text { value of statistical life } \\ x & \text { pollutant concentration } \\ Y_{0} & \text { baseline health endpoint rate }\end{array}$




\section{Chapter: Introduction}

Exposure to ambient air pollution has long been associated with adverse human health effects (U.S. EPA 2009; U.S. EPA 2013). The World Health Organization (WHO) reports ambient air pollution to be a leading risk factor for premature mortality and morbidity worldwide (WHO 2009). A recent study on the Global Burden of Disease (GBD) estimated 3.2 million and 152,000 deaths attributable to ambient PM and ozone exposure, respectively, in 2010 (Lim et al. 2012). Addressing the public health burden of ambient air pollution therefore remains a pressing scientific challenge.

Air quality is affected by emissions (anthropogenic and natural) and atmospheric transport and transformation processes that alter the composition of the atmosphere. Without the ability to control weather, controlling or reducing emissions is the only practical approach for managing air quality. Sensitivity analysis provides a means to estimate how air quality, or the impacts of air quality, might respond to changes in emissions. Sensitivity analysis in this context draws upon information from economics for estimation of the benefits and costs of control, epidemiology for establishing risk information, and atmospheric science and engineering for modelling the relationships between emissions and population exposure (Figure 1.1). This thesis situates itself at the interface between these disciplines and aims to develop policy and economic information from epidemiologic and atmospheric data to inform evidence-based decision-making.

$$
\frac{\Delta \text { Impact }}{\Delta \text { Emission }}=\frac{\Delta \$ \text { Benefit }}{\Delta \text { Health Outcome }} \cdot \frac{\Delta \text { Health Outcome }}{\Delta \text { Concentration }} \cdot \frac{\Delta \text { Concentration }}{\Delta \text { Emission }}
$$

Figure 1.1 Multi-disciplinary context of sensitivity analysis for air pollution decision-making. The first term on the right hand side of the equation is from economics for valuating health outcomes, the 
second term from epidemiology describing the risk of exposure to a specific pollutant, and the third term from science and engineering to link ambient pollutant concentrations with emissions.

Inclusion of epidemiologic and economic data in atmospheric models (referred to hereafter as chemical transport models, or CTMs) allows for estimating the health impacts of reducing emissions. With this type of information, policy items can be strategically compared to evaluate their effectiveness in reducing the health burden of air pollution. Before the research in this thesis was conducted, studies had included epidemiologic and economic data in atmospheric CTMs to estimate the health impacts of changes in emissions. The approaches employed, however, only yielded information for a limited number of emission sources and scenarios. Since emissions vary in their potential to influence population exposure - by species and by location and time of emission effectively reducing the health burden of air pollution relies on information at the level of each emission source or source sector. Novel modelling tools are available that provide this information on a source-by-source basis. Such tools are often referred to as reverse or backward modelling approaches and can attribute air quality metrics such as health impacts to sources of emissions.

Most broadly, the research presented in this thesis address how atmospheric CTMs can be used to inform air pollution and public health policy. This thesis seeks to answer this question by streamlining the integration of health impact assessment tools with atmospheric CTMs and uses sensitivity analysis to estimate how emission controls may influence public health. More specifically, the research included in this thesis addresses the following questions: 
- What are the spatial and temporal patterns of health metrics attributed to emissions?

- How do these behaviours differ between various health metrics and metrics based on attainment of standards?

- How do attributed health impacts/benefits respond to dynamic changes brought by progressive environmental policies?

- How does our understanding of epidemiology affect our estimates of source attributed health benefits?

- What do these findings mean for air quality and health policy?

The thesis presented here is structured based on a series of manuscripts that address the research questions listed above. To establish context for the research conducted as part of this thesis, Chapter 2 consists of a brief review of the extensive literature in air pollution epidemiology, health impact assessment, and atmospheric modelling. Chapter 3 outlines methods that are used in the subsequent research chapters to address the above questions, including discussions of atmospheric CTMs and sensitivity analysis methods. These introductory chapters are intended to provide a basic overview, while more details on the scientific context and methods are presented in later research chapters. The research chapters in this thesis consist largely of published papers (Chapters 4-7) or draft manuscripts that are not yet published (Chapter 8). These chapters are described in detail, below.

Chapter 4 of this thesis is original research that links air pollution-related mortality in North America to emission reductions in different locations and times within the same continent. This chapter uses backward sensitivity analysis in an atmospheric 
CTM (CMAQ-adjoint) for gas-phase species to link exposure to ozone and $\mathrm{NO}_{2}$ with precursor emissions. The reverse modelling approach implemented in CMAQ-adjoint is hereafter referred to as source attribution. Attributing air pollution-related mortality to sources of emissions provides insight for air quality policy as to which emission sources (and their reductions) are most influential on public health in Canada and the U.S.

Chapter 5 is original research that examines how reducing emissions on a sourceby-source basis (by location and time, as in Chapter 4) influences various types of air quality metrics in Canada and the U.S. This work considers two different ozone metrics those based on population exposure and those based on attainment of regulatory standards using the gas-phase CMAQ-adjoint.

Chapter 6 of this thesis is original research that examines how the public health benefits of emissions control change can be expected to change as the atmosphere becomes cleaner with progressively more stringent air quality management policies in the U.S. This work looks at ozone management through $\mathrm{NO}_{\mathrm{x}}$ emission control using CMAQadjoint. The role of atmospheric chemistry in ozone production is explored in the context of estimating the benefits of $\mathrm{NO}_{\mathrm{x}}$ control along the policy trajectory.

Chapter 7 consists of original research that, similarly to Chapter 6, examines how the public health benefits of emissions control change with continued air pollution abatement. This chapter is focused on Canadian air quality management and uses recent epidemiological data for Canada to examine the role of the shape of the epidemiologically-based concentration-response relationship in estimating the health 
benefits of progressive control policies. This work quantifies public health benefits of $\mathrm{NO}_{\mathrm{x}}$ control due to reduced ozone and $\mathrm{NO}_{2}$ exposure in the long-term.

Chapter 8 of this thesis is a commentary or opinion manuscript that presents a collection of research from the literature and this thesis. It discusses how the health benefits of emission reductions respond to dynamic environmental changes. The paper examines the roles of climate change, progressively more stringent emission reductions, advancing emission control technologies, and advancing epidemiologic evidence in estimating the health benefits of $\mathrm{NO}_{\mathrm{x}}$ emission control. 


\section{Chapter: Background and Literature Review}

This chapter reviews the literature linking ozone, nitrogen dioxide $\left(\mathrm{NO}_{2}\right)$, and particulate matter (PM) exposure to adverse health outcomes in North America. The focus of this thesis is on mortality as it constitutes the public health impact of air pollution with the largest monetary value. This section first provides an overview of the epidemiologic literature linking air pollution exposure and mortality, both in the shortterm and long-term. It then discusses the roles of epidemiologic and economic valuation data in conducting health impact assessments. It concludes with a discussion of how health impacts can be linked directly with emissions using atmospheric CTMs, with citations to works that have made significant advancements in this area of the literature.

\subsection{Epidemiologic Evidence Linking Air Pollution Exposure and Mortality}

The U.S. Environmental Protection Agency (U.S. EPA) released its latest Integrated Science Assessments (ISAs) on Ozone and Photochemical Oxidants in 2013 (U.S. EPA 2013) and on Particulate Matter in 2009 (U.S. EPA 2009). ISAs evaluate the state of the scientific literature on pollutant-related health and environmental outcomes, and conclude as to the likelihood of causal associations between exposures and outcomes. Findings of the most recent ISAs for ozone and PM indicate a breadth of health outcomes, including mortality, related to both short- and long-term exposures to various pollutants. A number of key studies included in the ISAs contribute to the weight of evidence in support of associations between ozone or PM exposure and mortality. These studies are discussed below. 
Epidemiology is one scientific discipline that informs ISAs in making their conclusions about causality. Epidemiologic studies quantify associations between exposures and health outcomes, and provide estimations of the excess risk of a specific health endpoint related to the exposure of interest. Measures of excess risk are often presented as concentration-response factors or effect estimates. Such measures quantify the increase in incidence of a health outcome for a specified change in pollutant concentration (such as a $\%$ increase in mortality rate per 10 ppb increase in ozone exposure). Two types of air pollution epidemiologic studies are discussed in this literature review. The first is a time-series design that assesses the short-term risk of exposure to air pollution. Time-series studies examine daily variability in population exposure (short-term changes in concentrations) and daily variability in the incidence of adverse health outcomes (such as mortality rates), and estimate associations between the two. The second type of study in air pollution epidemiology employs a cohort design that yields information on long-term risks associated with exposure to air pollution. In cohort studies, the incidence of a health outcome is compared amongst populations with different long-term exposures in order to derive associations as measures of increased or reduced risk. While other types of epidemiologic studies (e.g., panel, case-crossover, and case-control studies) have added to the body of evidence on air pollution exposure and adverse health outcomes, the focus of this literature review is on time-series and cohort studies conducted at the national scale commensurate with the modelling conducted for Canada and the U.S. in Chapters 4-7.

Epidemiologic studies range in their timeframe of analysis, spatial scales, statistical models employed, measures of association reported, and exposure time periods 
considered (including lag time between exposure and outcome for time-series studies).

As the focus of the proposed research is to integrate epidemiologic data with atmospheric CTMs at a national scale (or, at smallest, a regional scale), this section highlights largescale, multicity epidemiologic studies with broad spatial coverage in Canada and the U.S. for inclusion in atmospheric modelling. Since epidemiologic studies also differ in their reporting of effect estimates over various scales (e.g., for a $10 \mathrm{ppb}$ or interquartile range increase in ozone concentration), results described here are scaled to amount to the $\%$ change in mortality per $1 \mathrm{ppb}$ or $1 \mu \mathrm{g} / \mathrm{m}^{3}$ increase in concentrations of ozone $/ \mathrm{NO}_{2}$ or PM, respectively, to facilitate comparison.

\subsubsection{Epidemiologic Studies on Ozone}

Bell et al. (2004) is a widely cited, national epidemiologic study on the association between short-term ozone exposure and mortality in the U.S. The study employed time-series analysis across 95 communities in the U.S. over the period of 19872000. The combined strength of association across all communities for 24 -hr average ozone and non-accidental mortality was found to be $0.052 \% / \mathrm{ppb}$. This effect or risk estimate indicates a $0.052 \%$ increase in mortality risk per ppb rise in 24 -hr average ozone concentration. Statistically significant effects were observed for non-accidental mortality and cardiovascular and respiratory mortality. Bell et al. (2004) also reported effect estimates based on a 1-hr daily maximum averaging period of $0.034 \% / \mathrm{ppb}$ and for daily maximum 8 -hr average of $0.043 \% / \mathrm{ppb}$ for non-accidental mortality. In a later analysis by Bell and Dominici (2008), a similar effect estimate for 24-hr average ozone was reported using the same data set. 
Following the national-scale study conducted by Bell et al. (2004), Zanobetti and Schwartz (2008) examined the effect of daily maximum 8-hr average ozone on mortality in order to obtain information on the timing of death in susceptible individuals following exposure. Their analysis covered 48 cities in the U.S. over the period of 1989-2000. The authors found an overall effect of $0.053 \% / \mathrm{ppb}$ excess risk for daily maximum 8 -hr average ozone during summertime. The authors concluded that use of short-term, daily exposure measures is likely to underestimate the public health impact of ozone exposure overall, as they observed excess risk of mortality even up to 20 days after exposure.

Katsouyanni et al. (2009) reported an effect estimate for 1-hr maximum ozone of 0.076\%/ppb in the U.S. as part of the Air Pollution and Health: A Combined European and North American Approach (APHENA) project. Compared to multi-city studies such as those described below, meta-analyses (i.e., pooled analyses) that combine results from smaller studies have generally yielded higher pooled estimates (e.g., Bell et al. 2005; Ito et al. 2005; Levy et al. 2005).

Studies on the association between short-term ozone exposure and mortality in Canada have generally found larger effect estimates than those reported for the U.S. (e.g., Katsouyanni et al. 2009). A time series study by Burnett et al. (1998) estimated the associations between ozone and all-cause mortality for 12 Canadian cities, but did not report a combined estimate for Canada. A later study by Burnett et al. (2004) reported an overall $0.080 \%$ increase in non-accidental mortality per ppb increase in 1-hr maximum ozone concentration. The study again covered 12 cities in Canada over the period of 1981-1999. Considering the short averaging time (1-hr), the effect estimate found by Burnett et al. (2004) is a larger risk of mortality due to ozone exposure than most 
estimates reported for the U.S. A later study by Stieb et al. (2008) reported an effect estimate of $0.061 \% / \mathrm{ppb}$. The excess risk found in the study was derived with the purpose of developing a Canadian Air Quality Health Index based on a 3-hr moving average window of exposure.

As part of the APHENA study, an effect estimate of $0.083 \% / \mathrm{ppb}$ was reported for Canada for 24-hr average ozone and all-cause mortality (Katsouyanni et al. 2009). Both all-cause and respiratory mortality were significantly associated with ozone exposure, while the association with cardiovascular mortality was not at a statistically significant level.

Farhat et al. (2013) also reported effect estimates for short-term ozone exposure and three mortality outcomes (death from all causes, death from respiratory causes, and death from cardiovascular causes) in 12 Canadian cities over the period of 1980-2001. The study examined both ozone and $\mathrm{PM}_{2.5}$ as predictors of mortality in single-pollutant models. Effect estimates for all-cause mortality were reported to be $0.11 \% / \mathrm{ppb}$ increase in 1-hr maximum ozone, and ranged from 0.056 to $0.134 \% / \mathrm{ppb}$ for cause-specific mortality, with effects being larger for respiratory mortality.

Evidence linking long-term ozone exposure with mortality is less abundant in the epidemiologic literature. Jerrett et al. (2009) is a widely referenced study of the American Cancer Society (ACS) Cancer Prevention Study II (CPS-II) cohort that examined the risk of death due to long-term ozone exposure. The study examined 448,850 subjects in 96 urban areas of the U.S. from 1982-2000 at least 30 years in age at the time of enrollment. The study reported an overall statistically significant effect estimate of $0.104 \% / \mathrm{ppb}$ for 
daily 1-hr maximum ozone for mortality from respiratory causes. Unlike other causes of death, this effect was robust to inclusion of $\mathrm{PM}_{2.5}$ in a two-pollutant model. Around the same time, Krewski et al. (2009) reported a statistically significant effect of $0.2 \% / \mathrm{ppb}$ for summertime ozone and all-cause mortality in a single-pollutant model in the ACS CPS-II cohort using different exposure data.

A recent analysis of a population-based census cohort in Canada, the Canadian Census Health and Environment Cohort (CanCHEC) conducted by Crouse et al. (2015) examined the associations between long-term exposure to ozone, $\mathrm{NO}_{2}$, and $\mathrm{PM}_{2.5}$ and mortality in Canada. The cohort consisted of 3.6 million Canadians who filled out the 1991 long-form census. Subjects were followed up over 16 years. Ozone was found to increase the risk of non-accidental mortality in Canada by $0.188 \% / \mathrm{ppb}$ in the long-term after adjusting for confounding by $\mathrm{PM}_{2.5}$ and $\mathrm{NO}_{2}$. Ozone was found to be significantly associated with cause-specific mortality in the study in three-pollutant models. As $20 \%$ of Canadian households were required by law to fill out the census long-form, CanCHEC is truly representative of the mix of diversity in the Canadian population.

\subsubsection{Epidemiologic Studies on Nitrogen Dioxide}

While there is general agreement regarding a positive association of ozone with mortality in the literature, there is a lack of consensus for $\mathrm{NO}_{2}$. Findings of an independent and statistically significant association between $\mathrm{NO}_{2}$ exposure and mortality are more common in Canadian and European epidemiological studies, while more recent studies in the U.S. have found evidence of associations. 
Dominici et al. (2003) examined time-series data for all-cause mortality in 90 U.S. cities as part of the National Morbidity, Mortality, and Air Pollution Study (NMMAPS). The authors found no association between $\mathrm{NO}_{2}$ and all-cause mortality. A more recent, large-scale U.S. study found the opposite. Moolgavkar et al. (2013) conducted a time-series analysis for 108 U.S. cities on all-cause mortality and pollutant exposures from 1987-2000. The authors reported a positive and statistically significant association between $\mathrm{NO}_{2}$ and mortality in single-pollutant models. This finding was robust to inclusion of $\mathrm{PM}_{10}$ in a two-pollutant model, producing an effect estimate of 0.094\%/ppb. Moolgavkar et al. (2013) noted that they found a stronger association between $\mathrm{NO}_{2}$ and mortality than the associations observed for ozone and $\mathrm{PM}_{10}$. The authors suggested reconsideration of the emphasis on population health risk related to ozone and PM exposure in the U.S. in light of this. Even more recently, Turner et al. (2016), based on the ACS CPS-II, found $\mathrm{NO}_{2}$ to be an independent predictor of mortality. In single-pollutant models, $\mathrm{NO}_{2}$ was significantly associated with all-cause and circulatory mortality. Even after adjustment for $\mathrm{O}_{3}$ and $\mathrm{PM}_{2.5}$ in multi-pollutant models, the effect of $\mathrm{NO}_{2}$ was still significant for circulatory mortality. This large-scale study adds to the body of evidence in the U.S. supporting an $\mathrm{NO}_{2}$ effect on mortality.

Unlike the mixed conclusions regarding $\mathrm{NO}_{2}$ associations with mortality in the U.S., Canadian time-series studies have consistently found strong associations even after controlling for other confounding factors. A study by Burnett et al. (2004) estimated the association between $\mathrm{NO}_{2}$, among other criteria air pollutants, and mortality in Canada. The study employed time-series analysis over the period of 1981-1999 for 12 Canadian cities and reported an effect estimate for non-accidental mortality of $0.10 \% / \mathrm{ppb}$ of 3 -day 
average $\mathrm{NO}_{2}$ concentration. This association was sensitive to adjustment for ambient $\mathrm{PM}_{2.5}$ but remained statistically significant. Associations between short-term $\mathrm{NO}_{2}$ exposure and non-accidental mortality increased with inclusion of $\mathrm{PM}_{2.5}, \mathrm{PM}_{10-2.5}$, and $\mathrm{PM}_{10}$ each in multi-pollutant models.

A re-examination of the risk of short-term $\mathrm{NO}_{2}$ exposure in Canada by Brook et al. (2007) reaffirmed that of all pollutant species, $\mathrm{NO}_{2}$ had the strongest association with non-accidental mortality in Canada. The authors tested Canadian data to delineate whether the effect of $\mathrm{NO}_{2}$ on mortality was due to it being a surrogate for other species emitted from high-temperature combustion, such as VOCs and polycyclic aromatic hydrocarbons (PAHs), or whether other photo-chemically produced pollutants that are correlated with $\mathrm{NO}_{2}$ are responsible. The authors found that $\mathrm{NO}_{2}$ is the best single indicator of the spectrum of species freshly emitted from combustion sources, including $\mathrm{PM}_{2.5}$ and other toxic pollutants.

A later time-series study by Stieb et al. (2008) was conducted to develop a Canadian risk communication tool, the AQHI. In the study, a shorter averaging period for $\mathrm{NO}_{2}$ (3-hr average) was used to ascertain exposure to subjects. The AQHI was constructed using single pollutant models to estimate associations between short-term $\mathrm{NO}_{2}, \mathrm{O}_{3}$, and $\mathrm{PM}_{2.5}$ exposure and mortality. The authors reported an $\mathrm{NO}_{2}$ effect of $0.062 \%$ for daily 3-hr maximum $\mathrm{NO}_{2}$ concentration, similar to estimates reported for in the Air Pollution and Health: A European Approach (APHEA-2) study (Samoli et al., 2006). Stieb et al. concluded that their results were consistent with $\mathrm{NO}_{2}$ having an independent effect on mortality, but could not rule out that $\mathrm{NO}_{2}$ may be acting as an indicator of other 
species emitted from combustion sources whose effects on health are not yet fully understood.

Since these studies were published, the first analysis of the long-term effects of $\mathrm{NO}_{2}$ exposure on mortality in Canada was conducted using CanCHEC (Crouse et al., 2015). Crouse et al. found long-term $\mathrm{NO}_{2}$ exposure to be significantly associated with mortality due to various causes of death, including non-accidental and cardiovascular mortality. Effect estimates for $\mathrm{NO}_{2}$ alone using single pollutant models adjusting for confounders were similar to those reported for multi-pollutant models that controlled for $\mathrm{PM}_{2.5}$ and $\mathrm{O}_{3}$. An effect estimate of $0.129 \% / \mathrm{ppb}$ was reported for non-accidental mortality from the multi-pollutant model.

Meta-analyses and systematic reviews conducted on the collective literature on $\mathrm{NO}_{2}$ have reached various conclusions as the scientific evidence advances. A metaanalysis of epidemiologic studies worldwide conducted by Stieb et al. (2002) found an overall statistically significant effect of short-term $\mathrm{NO}_{2}$ exposure on all-cause mortality. The authors found a pooled effect estimate of $0.12 \% / \mathrm{ppb}$ for $24-\mathrm{hr}$ average $\mathrm{NO}_{2}$ based on single-pollutant models in the literature. However, the effect of $\mathrm{NO}_{2}$ on mortality became statistically insignificant with inclusion of other species in multi-pollutant models. A systematic review of worldwide scientific evidence linking $\mathrm{NO}_{2}$ to mortality conducted later in 2008 concluded that there is only moderate evidence of an association (Latza et al. 2008). In a more recent review of the literature, the Canadian Health Risks Assessment for Nitrogen Dioxide (Health Canada 2016) concluded that the collective scientific evidence is suggestive of a causal relationship between $\mathrm{NO}_{2}$ and chronic exposure mortality. The same review concluded that there is evidence for a likely causal 
relationship between short-term $\mathrm{NO}_{2}$ exposure and mortality (non-accidental, cardiopulmonary, cardiovascular, and respiratory causes of death). More specific disease outcomes, such as cardiovascular morbidity, generally have been shown to have larger and more consistent associations with $\mathrm{NO}_{2}$ (e.g., Samoli et al. 2006).

\subsubsection{Epidemiologic Studies on Particulate Matter}

Estimation and comparison of mortality risks of PM exposure in epidemiologic studies is complicated by the spatiotemporal variability in PM characteristics. Early epidemiologic studies identified associations between PM mass and mortality for various size fractions (e.g., total suspended particles or TSP, $\mathrm{PM}_{10}$, and $\left.\mathrm{PM}_{2.5}\right)($ Schwartz et al. 1996). However, in addition to particle size, chemical constituents of ambient PM differ in their risks of adverse health effects (HEI 2002). Bell et al. (2007) reported that at least seven constituents make up more than $79-85 \%$ of ambient $\mathrm{PM}_{2.5}$ by mass in the U.S. These constituents, in typical order of abundance, are organic carbon, sulphate, nitrate, ammonia, elemental carbon, sodium ion, and silicon (Bell et al. 2007). With monitoring data now available on PM constituents, large-scale studies have begun to investigate the health risks of exposure to specific PM constituents (e.g., Krall et al. 2013). While such information offers insight into the toxicity of different types of particles originating from various source types, this section focuses on effect estimates for PM mass of small size fractions, i.e., $\mathrm{PM}_{2.5}$ or particles with an aerodynamic diameter less than $2.5 \mu \mathrm{m}$.

A time-series study by Zanobetti and Schwartz (2009) examined the association between $\mathrm{PM}_{2.5}$ and $\mathrm{PM}_{10}$ exposure and a spectrum of health outcomes, including mortality. The authors reported an effect estimate of $0.098 \% / \mu \mathrm{g} / \mathrm{m}^{3}$ for all-cause 
mortality across 48 U.S. cities due to $\mathrm{PM}_{2.5}$ exposure over the period of 1999-2005. Associations were stronger for $\mathrm{PM}_{2.5}$ than for coarser particles for all health outcomes assessed in the study. In an earlier time series study, Dominici et al. (2007) also found a stronger association for $\mathrm{PM}_{2.5}$ than $\mathrm{PM}_{10}$ for all-cause and cardiorespiratory mortality in the NMMAPS. The authors reported effect estimates of $0.029 \% / \mu \mathrm{g} / \mathrm{m}^{3}$ (for all-cause mortality) and $0.038 \% / \mu \mathrm{g} / \mathrm{m}^{3}$ (for cardiorespiratory mortality) for $\mathrm{PM}_{2.5}$ over a longer time period, from 1987-2000. Peng et al. (2005) found, in their analysis of the NMMAPS, that effect estimates for $\mathrm{PM}_{10}$ were highest in summer. The authors concluded that the seasonality of mortality response to $\mathrm{PM}_{10}$ yields insight into the toxicity of PM constituents, which vary by season.

Effect estimates reported in the literature suggest that long-term PM exposure has the potential to incur a substantially larger public health burden than short-term exposure. Few large-scale cohort studies on the risks of long-term $\mathrm{PM}_{2.5}$ exposure have been conducted at the national level commensurate with the research of this thesis. A small number of studies in the U.S. have been conducted on the ACS CPS-II cohort to examine the association between long-term PM exposure and mortality. In an earlier analysis, Pope et al. (2002) found an increased risk of all-cause mortality equal to $0.6 \%$ per $\mu \mathrm{g} / \mathrm{m}^{3}$ increase in annual average $\mathrm{PM}_{2.5}$. Associations between $\mathrm{PM}_{2.5}$ exposure and all-cause, cardiopulmonary, and lung cancer mortality were all statistically significant in singlepollutant models. A later re-analysis of the same cohort by Krewski et al. (2009) over a period of follow up from 1982-2000 found that long-term risks for all-cause mortality range between $0.3-0.6 \% / \mu \mathrm{g} / \mathrm{m}^{3}$. 
Findings from the APHENA study indicate that risks of mortality associated with short-term PM exposure are comparable between the U.S. and Europe, but are consistently higher for Canada (Samoli et al. 2008). Burnett et al. (2000) conducted a time-series study in Canada and reported an effect estimate of $0.120 \% / \mu \mathrm{g} / \mathrm{m}^{3}$ for mortality from $\mathrm{PM}_{2.5}$ exposure. The study was based on 8 of Canada's largest cities over 1986-1996. In a revised analysis of this time-series study, Burnett and Goldberg (2003) reported effect estimates of $0.110-0.117 \% / \mu \mathrm{g} / \mathrm{m}^{3}$. A later Canadian time-series study by Stieb et al. (2008) used a 3-hr window of exposure relevant to development of the AQHI and reported an association of $0.13 \% / \mu \mathrm{g} / \mathrm{m}^{3}$ for non-accidental mortality and $\mathrm{PM}_{2.5}$ exposure. Effect estimates for a recent Canadian time-series study conducted over a 20year period from 1981-2001 by Farhat et al. (2013) were similar in magnitude, ranging from $0.098-0.177 \% / \mu \mathrm{g} / \mathrm{m}^{3}$. Effect estimates were larger for cardiovascular mortality than all-cause mortality.

The first national-level cohort study on the long-term effects of $\mathrm{PM}_{2.5}$ exposure in Canada was conducted by Crouse et al. (2012). The authors tracked mortality within a cohort of 2.7 million subjects over the period 1991-2001 to assess their risk of various types of mortality due to long-term $\mathrm{PM}_{2.5}$ exposure. The cohort consisted of $20 \%$ of the Canadian population who were required to fill out the 1991 long-form census. The study reported associations of $0.95 \%$ and $1.4 \%$ increases in non-accidental mortality risk per $\mu \mathrm{g} / \mathrm{m}^{3}$ increase in annual average $\mathrm{PM}_{2.5}$ concentration. The authors did not assess confounding by other pollutants in the ambient mixture such as ozone or $\mathrm{NO}_{2}$, but did so for socioeconomic and demographic characteristics at the community scale. The cohort of 1991 long-form census respondents analyzed by Crouse et al. (2012) was later named the 
Canadian Census Health and Environment Cohort (CanCHEC) and analyzed in a subsequent study by Crouse et al. (2015) for 16 years of follow up from 1991-2006. This later study used exposure surfaces for ozone and $\mathrm{NO}_{2}$ in addition to $\mathrm{PM}_{2.5}$ to assess risks of each pollutant individually and in combination. The authors found a smaller association between $\mathrm{PM}_{2.5}$ and mortality compared to the earlier study. A $1 \mu \mathrm{g} / \mathrm{m}^{3}$ increase in long-term $\mathrm{PM}_{2.5}$ exposure was associated with a $0.22 \%$ increased risk of non-

accidental mortality. The effect of $\mathrm{PM}_{2.5}$ was statistically significant in both single and multi-pollutant models adjusted for confounding by ozone and $\mathrm{NO}_{2}$.

\subsection{Epidemiologic and Valuation Data in Health Impact Assessment}

Epidemiologic findings from time-series and cohort studies are routinely applied in health impact assessment to estimate the societal or public health burden of air pollution exposure. Health impact assessment aims to estimate the public health burden or impact of a change in air quality, either as an aggregate count of health outcomes, or in monetary terms as an economic benefit (or cost, depending on the direction of the change in air quality) to society. Health impact assessment is often performed for predefined air quality scenarios (such as attaining a new standard), and in this way, health outcomes are not linked to emissions themselves. Linkage of health impacts to emissions requires integration of health impact assessment methods with atmospheric CTMs (the topic of section 2.3).

Health impact assessment tools exist in both Canada and the U.S. for use in regulatory applications, and use epidemiologic data relevant to each country. The Air Quality Benefits Assessment Tool (AQBAT; Judek et al. 2006) and the Environmental Benefits Mapping and Analysis Program (BenMAP) are the respective tools for Canada 
and the U.S., developed by Health Canada and the U.S. EPA for this purpose. The governing equations applied in health impact assessment are discussed in section 3.3. It is noted that quantified health impacts can be expressed as a number of health outcomes (e.g., 1,000 deaths) averted due to an improvement in air quality, or as a monetary value (e.g., \$1,000). The latter applies a monetary valuation measure to convert from an aggregate mortality count to a dollar value. For mortality, the valuation measure is the value of statistical life (VSL), which is a willingness-to-pay for a reduction in risk of premature death (Alberini et al. 2006). Typical values used for VSLs in the U.S. and Canada are discussed in section 3.3.

\subsubsection{Air Quality Health Impact Assessment Studies}

Few studies have employed health benefit assessment tools at the national level commensurate with the scale of the proposed research. Such studies quantify the health impact of a specified change in air quality (i.e., from one scenario to another), or else use a reference air quality scenario of zero or background concentrations to estimate the overall burden of exposure to ambient pollution. The latter implies that associations between exposures and health outcomes derived from epidemiologic studies hold true at low levels of pollution (i.e., a no-threshold or linear response).

Scenario-based studies have estimated the health benefits of a shift in air quality to attain various air quality standards in the U.S. Hubbell et al. (2005) is one such study that used BenMAP to estimate the health benefits of reducing nation-wide ozone concentrations from 2000-2002 to levels in compliance with an ozone standard of 0.08 ppm. The monetized public health benefit of attaining this ozone standard was estimated to be $\$ 4.9-5.7$ billion/year. This estimation included an average annual mortality 
reduction of 800 deaths valued at approximately $\$ 5.2$ billion/year. Reduction in premature mortality is therefore a large contributor to the overall monetized public health benefits of improving air quality. More recently, Berman et al. (2012) assessed the U.S. health benefits related to attaining the $75 \mathrm{ppb}$ ozone standard of 2008 . The authors applied a spectrum of effect estimates from the epidemiologic literature, including that of Bell et al. (2004A) and Ito et al. (2005) in their health impact assessment. The study found 1,400-2,500 deaths per year averted between 2005-2007 with nationwide attainment of a $75 \mathrm{ppb}$ standard. The study also estimated benefits of stricter regulatory thresholds, and found that nation-wide ozone concentrations below $60 \mathrm{ppb}$ avert up to 8,000 premature deaths/year.

Multi-pollutant health impact assessments have found that the public health burden associated with $\mathrm{PM}_{2.5}$ exposure far exceeds that of ozone. A report on the Global Burden of Disease (GBD) Study by Lim et al. (2012) estimated the mortality burden of ambient air pollution exposure worldwide. The authors estimated 3.2 million and 152,000 deaths/year attributable to ambient PM and ozone exposure, respectively. Anenberg et al. (2010) used atmospheric modelling for exposure data worldwide in their estimation of the global health burden of ozone and $\mathrm{PM}_{2.5}$ exposure. The authors applied long-term exposure effect estimates from Jerrett et al. (2009) and Krewski et al. (2009) and found that 35,000 deaths/year in North America for ozone, and 141,000 deaths/year for $\mathrm{PM}_{2.5}$ could be attributed to anthropogenic air pollution. Later, Fann et al. (2012B) conducted a study to assess the public health burden of mortality related to short- and long-term ozone and $\mathrm{PM}_{2.5}$ exposure in the U.S. An estimated 130,000 deaths/year and 320,000 deaths/year were attributed to short- and long-term $\mathrm{PM}_{2.5}$ exposure based on 2005 
concentrations. For ozone, the authors estimated 4,700 and 19,000 deaths/year, related to short- and long-term exposure, respectively.

\subsection{Health Impacts of Emission Reductions}

One of the challenges in air quality policy is to directly quantify the benefits of emission reductions necessary for air quality improvements. Assessing health impacts related to population exposures requires estimates of epidemiologic associations, population exposure, and economic valuation measures. Further linking exposures to emissions requires information on atmospheric processes that dictate transport and transformation of species emitted at a source to receptor populations. Quantifying the impacts of reducing emissions therefore relies on atmospheric CTMs and is often categorized as a sensitivity analysis question. For secondary species such as ozone and inorganic PM, the chemical composition of the atmosphere affects the potential for pollutant formation, and therefore the sensitivity or response of concentrations to precursor emissions changes nonlinearly. Comprehensive representations of atmospheric physics, chemistry, and particle dynamics in atmospheric CTMs are therefore essential in estimating sensitivities of these species' concentrations (or other metrics such as health outcomes) to emission reductions. This section outlines examples of studies that create links between emissions and health impacts by inclusion of epidemiologic and economic valuation data in atmospheric CTMs. It should be noted that sensitivity analysis methods are not discussed here; rather, detailed information is provided in section 3.2 , and a detailed description of the chemistry of ozone formation is presented in Appendix A.

Traditionally, health impacts have been related to emissions using forward sensitivity analysis (discussed in section 3.2) in atmospheric CTMs. One such example is 
a study by Anenberg et al. (2009) that used a global atmospheric CTM to estimate the sensitivity of ozone-related mortality to precursor emission reductions. The study applied a U.S.-based effect estimate from Bell et al. (2004A) to quantify mortality associated with changes in short-term ozone exposure worldwide. The authors used a series of model simulations for various emission scenarios (baseline emissions and scenarios where emissions from a single continent were each reduced by $20 \%$ ) to estimate changes in ozone concentrations worldwide. In doing so, the study aimed to isolate the influences of emissions in each source region on the worldwide ozone public health burden. The study reported 21,800 averted acute deaths in the northern hemisphere per year due to a worldwide $20 \%$ reduction in anthropogenic emissions of $\mathrm{NO}_{\mathrm{x}}$, non-methane volatile organic compounds (VOCs), and CO emissions.

West et al. (2006) applied a similar approach and effect estimate from Bell et al. (2004A) in a global atmospheric CTM to estimate the sensitivity of ozone-related mortality to methane emission reductions. The authors predicted an annual reduction in global mortality of 30,000 deaths by 2030 due to a $20 \%$ reduction in methane emissions worldwide. The study examined the cost-effectiveness of mitigating methane emissions, and found a benefit-to-cost ratio of 4:1 after applying a uniform VSL of \$1 million per statistical life around the globe. It is noted that VSL estimates vary significantly depending on the geographic region of consideration (Alberini et al. 2006), and the estimate applied in this global study is much lower than those used in North America (U.S. EPA 2010).

A more recent study by Tagaris et al. (2010) used formal sensitivity analysis in an atmospheric CTM over the continental U.S. to relate $\mathrm{PM}_{2.5}$ and ozone mortality to 
precursor emissions under the influence of climate change. The authors also applied the short-term effect estimate from the widely used Bell et al. (2004A) study for ozone. The effect estimate for $\mathrm{PM}_{2.5}$ was taken from Pope et al. (2002) as a measure of association between long-term exposure and mortality. The authors reported up to 270 or 400 deaths/year averted per U.S. state from reduced $\mathrm{PM}_{2.5}$ exposure due to domain-wide $10 \%$ reduction in $\mathrm{NO}_{\mathrm{x}}$ or $\mathrm{NH}_{3}$ emissions, respectively. State-specific ozone-related mortality was most sensitive to $\mathrm{NO}_{\mathrm{x}}$ emission reductions. The authors estimated that a domainwide $10 \%$ reduction in $\mathrm{NO}_{x}$ would reduce premature mortality by up to 40 deaths/year in a given state.

The studies discussed so far employed forward sensitivity analysis to estimate the response of health outcomes to reductions in emissions. Reverse or adjoint sensitivity analysis offers information complementary to that provided by forward methods, and is the method employed in Chapters 4-7 of this thesis. Forward and adjoint sensitivity analysis are discussed in detail in section 3.2. Given the novelty of the adjoint method and its application in modelling of air pollution health impacts, no study had been conducted employing this method at the onset of Chapter 4 of this thesis. Since publication of Chapters 4-7 of this thesis, this area of research has been active and a series of studies employing adjoint sensitivity analysis have been published.

A study by Koo et al. (2013) investigated the influence of aviation emissions on $\mathrm{PM}_{2.5}$-related mortality using a global atmospheric CTM (the GEOS-Chem model). The study applied a linear concentration-response function derived in Barrett et al. (2012), which was equivalent to an effect estimate of $1 \% / \mathrm{ug} / \mathrm{m}^{3}$. The authors presented a matrix of source-receptor relationships, and estimated the influences of emissions from each 
continent on the total $\mathrm{PM}_{2.5}$-related mortality count worldwide. Aviation emissions from North America were found to contribute the most to worldwide mortality (an averted 750 worldwide deaths per year for a 10\% reduction). Emissions in other source regions had influences ranging from 500-700 deaths/year for a $10 \%$ reduction.

A later study by Lee et al. (2015) also applied the adjoint of GEOS-Chem in a health impact assessment of $\mathrm{PM}_{2.5}$ precursor emission reductions. The study linked global premature mortality related to ambient $\mathrm{PM}_{2.5}$ exposure to precursor emission reductions originating from different source locations around the world. The authors found ammonia, organic carbon (OC), and black carbon (BC) to have the largest influences on global $\mathrm{PM}_{2.5}$-related mortality, and emissions in Eastern Europe and Asia to be of highest consequence to global health. On a marginal emissions basis of $1 \mathrm{~kg}$ per $\mathrm{km}^{2}$ per year, ammonia was found to result in up to 15 premature deaths per year worldwide, while OC and $\mathrm{BC}$ were linked to a maximum of 6.3 and 5.2 deaths per annum, respectively. With the exception of Eastern Europe and Central Asia, overall, carbonaceous primary particles had the largest sensitivities.

A study by Turner et al. (2015) published the same year examined source-receptor relationships between $\mathrm{BC}$ emissions and $\mathrm{PM}_{2.5}$-related premature mortality in the U.S. using the $\mathrm{BC}$ adjoint of CMAQ. The authors reported an overall $\mathrm{BC}$ health burden in the U.S. of 12,200 premature deaths per year. The study reported relative contributions of the U.S. states, both by emission quantities and public health impacts, for comparing the estimated damage from specific sources with the intensity of the emission source. California's BC emissions were found to account for 2,000 premature deaths per year in the U.S., followed by the states of Illinois, New York, and Texas, each with an estimated 
burden of around 800 deaths. The authors found that per unit of $\mathrm{BC}$ emissions, onroad diesel $\mathrm{BC}$, nonroad mobile $\mathrm{BC}$, and onroad gasoline $\mathrm{BC}$ had the highest public health damages.

Many of the studies discussed so far have reported health benefits of relative reductions in emissions (i.e., a percent reduction with reference to a baseline). A more common unit of analysis in environmental economics is the health benefit of reducing 1 ton of emissions, termed the marginal benefit or marginal damage. Scaling sensitivities in this way allows for comparison of the same hypothetical unit of emission reduction across different locations and times. Studies in the environmental economics literature commonly use simplified models (e.g., dispersion or response-surface models) for estimating the marginal benefit of reducing emissions.

Fann et al. (2009) estimated $\mathrm{NO}_{\mathrm{x}}$ marginal benefits averaging $\$ 10,000-15,000 /$ ton in the U.S., depending on the source sector, using reduced-form modelling. The study focused on 9 urban areas of the U.S. and the marginal benefit estimates quantified the averted mortality due to reduced $\mathrm{PM}_{2.5}$ exposure resulting from 1 ton reduction in emissions from various source types. For $\mathrm{SO}_{\mathrm{x}}$ emissions, benefits-per-ton averaged $\$ 15,000-29,000 /$ ton nationally. Benefits-per-ton for primary carbon were largest, averaging $\$ 460,000-720,000 /$ ton, while benefits-per-ton for $\mathrm{NH}_{3}$ averaged $\$ 38,000$ 95,000/ton. A later study by Fann et al. (2012A) reported average $\mathrm{NO}_{\mathrm{x}}$ marginal benefits of $\$ 3,700-\$ 4,500 /$ ton (based on $\mathrm{PM}_{2.5}$ mortality again) for the entire U.S using CTMbased source apportionment. Their estimates for primary $\mathrm{PM}_{2.5}$ across the U.S. ranged from $\$ 70,000-\$ 450,000 /$ ton, depending on source sector. For $\mathrm{SO}_{2}$, benefits-per-ton ranged from $\$ 20,000-360,000 /$ ton, though most sectors were on the lower end of this 
range. Significantly lower marginal benefits have been estimated by Muller and Mendelsohn (2009) based on simplified dispersion models that do not account for complex atmospheric chemistry, averaging $\$ 260 /$ ton for $\mathrm{NO}_{\mathrm{x}}$ in the U.S. 


\section{Chapter: Methods}

This thesis aims to establish links between sources of emissions and human health effects by incorporating data from health benefit assessment tools into atmospheric CTMs. Traditionally, atmospheric CTMs have been used to relate anthropogenic emissions to concentrations, which can then be extended to public health effects using relevant epidemiologic and economic data used in health impact assessment. This section provides a brief overview of the methods employed in atmospheric CTMs and health impact assessment, separately in their respective fields, and integrated together in the context of Chapters 4-8 of this thesis. It is noted that each research chapter of this thesis contains a detailed methodology section as part of the published or draft manuscript. This section therefore provides a broader overview of the methods that apply to the research chapters of this thesis, but is not intended to be comprehensive to avoid redundancy.

\subsection{Atmospheric Chemical Transport Models}

Atmospheric CTMs simulate transport and physical and chemical transformation processes that act on air pollutants of natural and anthropogenic origin and alter the composition of the atmosphere. They are gridded models of varying resolutions and can be global, regional, or local in extent. Such models require spatially and temporally resolved emissions and meteorological data as model inputs. Emissions are transported in an atmospheric CTM using meteorological data, and transformed on the path from their sources to receptors. The output of a traditional atmospheric CTM is a field of concentration estimates for all model species and all grid-cell locations at regular time intervals (e.g., hourly concentrations) over the simulation period. Atmospheric CTMs and their derivative sensitivity analysis forms (discussed in section 3.2) are ideal tools for 
answering "what-if" policy questions that would be expensive or impractical to test in real life.

Atmospheric CTMs solve a version of the atmospheric diffusion equation (ADE; Equation 3.1, below) for each grid cell in the modelling domain. The ADE tracks the evolution of the concentration field over time for a set of initial and boundary conditions (Jacob 2007),

$$
\frac{\partial C_{i}}{\partial t}=-\nabla \cdot\left(\boldsymbol{u} C_{i}\right)+\frac{1}{\rho} \nabla \cdot\left(\rho \boldsymbol{K} \nabla C_{i}\right)+R_{i}+E_{i}
$$

In Equation 3.1, $C_{i}$ is the mixing ratio of species $i, u$ is the wind velocity field, $\rho$ is the air density, $K$ is the vector diffusivity coefficient, $R_{i}$ is the rate of chemical reaction of species $i$, and $E_{i}$ is the emission rate of species $i$. The first two terms on the right hand size of Equation 3.1 describe transport of species $i$ by advection and diffusion, respectively. The third term, $R_{i}$, includes both chemical production and loss of species $i$. For pollutants like ozone, a number of chemical reactions are accounted for in this term (refer to Appendix A for a detailed explanation of ozone chemistry). It is noted that Equation 3.1 is a simplified form of the ADE in which loss through dry deposition is included as part of the boundary condition for vertical diffusion.

In operator form, Equation 3.1 is equivalent to Equation 3.2, below, where $\alpha$ is a vector of model input parameters, and $M$ is a nonlinear model operator matrix that maps from the input vector onto the output domain,

$$
\boldsymbol{C}=\boldsymbol{M} \alpha
$$


Atmospheric CTMs have been developed with different representations of the scientific processes in Equation 3.1, and for different geographic scales. A brief overview of the atmospheric CTM used in Chapters 4-8 of this thesis - a widely used regional air quality model developed by the U.S. EPA - is presented in the following section.

\subsubsection{Community Multiscale Air Quality Model (CMAQ)}

The proposed research uses the U.S. EPA's Community Multiscale Air Quality model (CMAQ) and its derivative sensitivity analysis tools. CMAQ is a widely used atmospheric CTM that has undergone continuous development by researchers in the broader air quality modelling community. CMAQ is a comprehensive modelling system with a CTM processor (CMAQ Chemistry Transport Model, or CCTM) and additional processors to prepare model inputs for running CCTM. CCTM has six scientific modules that span over the range of atmospheric transport processes, gas-phase and aerosol chemistry, deposition, and cloud formation processes needed for solving the ADE in Equation 3.1. A Photolysis Rate Processor (JPROC) is used to calculate photolysis rates for photochemical reactions. The Initial Conditions Processor (ICON) and Boundary Conditions Processor (BCON) are used to create the initial and boundary conditions for which the ADE is solved (Byun and Schere 2006). Meteorological inputs for CMAQ are generated using the Weather Research and Forecasting (WRF) model (Skamarock et al. 2005), and processed using the Meteorology Chemistry Interface Processor (MCIP). Illustration of the CMAQ modelling system is shown in Figure 3.1, below. Emission inputs for CMAQ are generated using the Sparse Matrix Operator Kernel Emissions (SMOKE) model (UNC Institute for the Environment, 2009) based on the National 
Emission Inventory (NEI) for the U.S. and the National Pollutant Release Inventory (NPRI) for Canada.

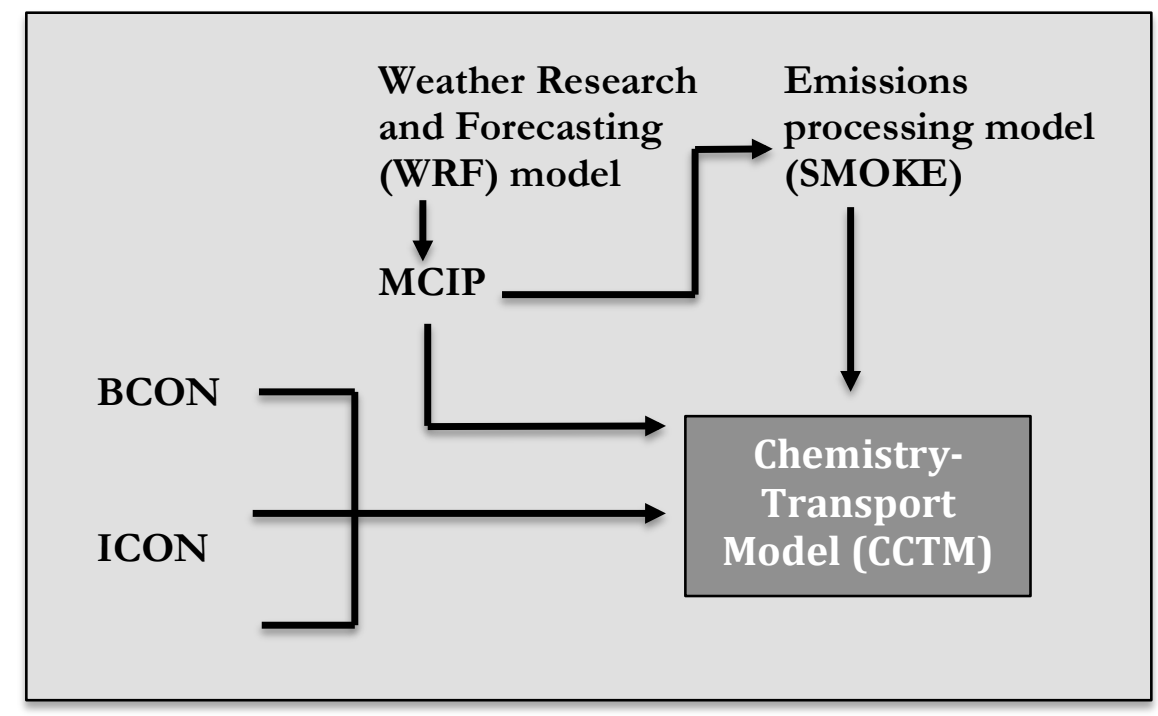

Figure 3.1 CMAQ Input Processors and CCTM (adapted from Byun and Schere 2006)

\subsection{Sensitivity Analysis Methods}

Air quality management often draws upon sensitivity information. Examples of such information might include estimates of how reducing anthropogenic emissions would impact air quality or other measures related to air quality (such as public health impacts). Sensitivities in this context refer to derivatives of air quality metrics, such as concentration or public health impacts with respect to human activity or behaviours. In an atmospheric CTM, this amounts to derivatives of model output(s) with respect to model input(s), such as the response of concentration to a change in emissions, or $\delta C / \delta E$. Atmospheric CTMs are appropriate tools for answering sensitivity questions because they link sources of emissions to receptors whose air quality is of interest. Formal methods of 
sensitivity analysis have been developed in atmospheric CTMs that estimate spatiotemporal derivatives or sensitivities of model output(s) to input(s).

Two categories of sensitivity analysis are often undertaken using atmospheric CTMs to evaluate or predict the air quality implications of reducing emissions. Forward sensitivity analysis and adjoint or backward sensitivity analysis are two modelling approaches that yield different, yet complementary information. Forward sensitivity analysis is a traditional approach to sensitivity analysis that seeks to answer the following question: how would a widespread (e.g., sector-wide or regional) reduction in emissions influence air quality in different locations? Forward sensitivity analysis is a sourceoriented approach that links a change in one model input (such as emissions of $\mathrm{NO}_{\mathrm{x}}$ collectively from all mobile sources, in a specific region) to each model output (i.e., concentrations at all grid cell locations, at all model time steps). In contrast, adjoint sensitivity analysis is a more recent approach that seeks to answer the reverse type of question: how would a collective air quality metric defined over many receptors (e.g., the average concentration in a defined region) respond to changes in emissions from each source (i.e., emissions in each location, at each time). This reverse method instead links changes in one model output to many model inputs. It does so by starting at the receptor and tracing backwards in time to estimate how each model input affects the receptor, and is therefore receptor-oriented. This section provides an overview of the equations that govern both sensitivity analysis approaches in atmospheric CTMs.

\subsubsection{Forward Sensitivity Analysis}

Forward sensitivity analysis in atmospheric CTMs is governed by a perturbed version of Equation 3.1. For an incremental change in a model input parameter (in this 
case, emissions or $\delta E_{i}$ ), a corresponding change in concentration, $\delta C_{i}$, is given by (Hakami et al. 2007),

$$
\frac{\partial \delta C_{i}}{\partial t}=-\nabla \cdot\left(\boldsymbol{u} \delta C_{i}\right)+\frac{1}{\rho} \nabla \cdot\left(\rho \boldsymbol{K} \nabla \delta C_{i}\right)+\boldsymbol{F}_{i} \delta \boldsymbol{C}+\delta E_{i}
$$

where $F_{i}$ is $i$-th row of the Jacobian of the chemistry operator for species $i$, and all other parameters are as defined in Equation 3.1. In operator form, the linearized operator $L$ maps the perturbed model inputs, $\delta \alpha$, onto the domain of perturbed model outputs, $\delta C$,

$$
\delta \boldsymbol{C}=\boldsymbol{L} \delta \alpha
$$

In Equation 3.4, $L$ is a linear operator and the Jacobian of the model operator matrix, $M$ (Equation 3.2), and of size $m \times n$, where $m$ is the number of model outputs and $n$ is the number of model inputs. Equations 3.3-3.4 define the tangent linear model (TLM) or the direct decoupled method (DDM) operator used in forward sensitivity analysis. Equation 3.3 is the system of equations solved for each model species, $i$, in the TLM or DDM (Dunker et al. 1981; Yang et al. 1997). It is noted that one simulation using the TLM/DDM yields a single column, $j$, of the operator $L$ in Equation 3.4, which contains the sensitivities of all model outputs to one model input, or $\delta C_{i=1, m} / \delta \alpha_{j}$. The TLM/DDM are direct sensitivity approaches that are implemented in CMAQ to calculate local sensitivities or derivatives with respect to a single (or a few) model input(s).

\subsubsection{Adjoint Sensitivity Analysis}

Adjoint sensitivity analysis is a reverse or complementary approach to forward sensitivity analysis. Rather than estimate sensitivities of all model outputs with respect to one input parameter, the adjoint method instead estimates sensitivities of a single metric 
based on model outputs (or single model output) to all model inputs. This single metric is defined based on the type of question one seeks to answer using adjoint modelling and can be devised to address a policy-relevant metric, such as attainment of an air quality standard or a metric quantifying public health impacts of air pollution exposure. This metric of interest is termed the adjoint cost function, and can be any function of concentration, $g(C)$, integrated over any choice of temporal period $(t)$ and spatial extent $(\omega)$

$$
J=\int_{t} \int_{\omega} g(C, t, \omega) d \omega d t
$$

The adjoint variable, $\lambda_{i}$ for species $i$, is the derivative of the adjoint cost function, $J$, with respect to concentration at any given time through all model processes,

$$
\lambda_{i}=\frac{\partial J}{\partial C_{i}}
$$

The governing equation for the adjoint model tracks the evolution of the adjoint variable, $\lambda_{i}$, over time, as in Equation 3.7,

$$
-\frac{\partial \lambda_{i}}{\partial t}=\boldsymbol{u} \cdot \nabla \lambda_{i}+\nabla \cdot\left(\rho \boldsymbol{K} \nabla \frac{\lambda_{i}}{\rho}\right)+{F_{i}}^{T} \lambda+\varphi_{i}
$$

Equation 3.7 bears some similarity to the ADE and can be derived from Equation 3.3 using Lagrange multipliers and integration by parts. $F_{i}{ }^{T}$ is the $i^{\text {th }}$ column of the Jacobian of the chemistry operator, $F$, transposed. The adjoint forcing term, $\varphi_{i}$, is synonymous to the emission term, $E_{i}$, in the forward model (Equation 3.1). Adjoint forcing terms are injected into the adjoint model and drive the evolution of the adjoint 
variables over time, as defined by Equation 3.7, in the same way that emissions are injected in the forward model and drive the evolution of concentration over time in Equation 3.1. The adjoint forcing term is an input to the adjoint model and is dependent upon the choice of adjoint cost function (Equation 3.5). Mathematically, the adjoint forcing term is the derivative of the local cost function, $g\left(C_{i}, t, \omega\right)$, with respect to concentration,

$$
\varphi_{i}=\frac{\partial g\left(C_{i}, t, \omega\right)}{\partial C_{i}(t, \omega)}
$$

In adjoint sensitivity analysis, the derivative of the adjoint cost function, $J$, with respect to all model inputs is sought. In operator form, the transposed linear operator, $L^{T}$, maps the adjoint forcing term, $\varphi$, onto the domain of adjoint gradients,

$$
\lambda=\nabla_{C} J=L^{T} \nabla_{C_{f}} J=L^{T} \varphi
$$

It is noted that in Equation 3.9, $\varphi$ is also denoted as $\nabla_{C_{f}}$, or the derivative of $J$ with respect to $C$ at the start of the adjoint simulation. Since $\lambda$ is calculated backwards in time in the adjoint model, the start of the time step refers to the final concentration, or the concentration at the latest point in time. Mathematically, the adjoint gradient, or $\nabla_{\alpha} J$, is the product of $\nabla_{\alpha} C$ (or $\partial C / \partial \alpha$ ), transposed, and the adjoint variable, $\lambda$,

$$
\nabla_{\alpha} J=\left(\nabla_{\alpha} C\right)^{T} \nabla_{C} J=\left(\nabla_{\alpha} C\right)^{T} \lambda
$$

As described above, the adjoint method is a direct sensitivity approach that calculates local sensitivities or derivatives. An adjoint tool for CMAQ was developed for gas-phase species in 2007 (Hakami et al. 2007) and is used in Chapters 4-7 of this thesis. 
Ongoing efforts are underway for developing an adjoint to CMAQ for particle-phase processes.

\subsection{Estimating Air Pollution Health Impacts}

Sensitivity analysis of air pollution health impacts requires integration of data from health impact assessment into atmospheric CTMs. This section describes the health impact function and its implementation in adjoint sensitivity analysis.

\subsubsection{The Health Impact Function}

In health impact assessment, the public health impact resulting from a change in air quality is estimated using the health impact function. The health impact function combines elements from epidemiology with population and mortality data to estimate the number of deaths attributable to a change in air pollution exposure. It can be evaluated for any health outcome of choice (e.g., all-cause mortality, non-accidental mortality, cause-specific mortality, or acute morbidity) provided that relevant epidemiological and population data are available. An example of a health impact function is shown below,

$$
\Delta M=M_{0} P\left(1-e^{-\beta \Delta C}\right)
$$

where $\Delta M$ is the change in mortality attributable to a change in pollutant exposure $\left(\mathrm{yr}^{-1}\right)$, $M_{0}$ is the baseline annual mortality rate (a fraction of the population; $\mathrm{yr}^{-1}$ ), $P$ is the population, $\beta$ is the effect estimate or concentration-response factor (the $\%$ change in $M_{0}$ per ppb), and $\Delta C$ is the change in ambient concentration of the pollutant. It is noted that this form of health impact function implies a concentration-response function that is linear in concentration. Alternate forms of the health impact function exist in the 
literature for a linear concentration-response function, but are similar to that shown here, and provide the same estimate of $\Delta M$ for any practical change in concentration. Other forms based on non-linear concentration-response functions that would follow different formulations, such as that for a supralinear curve, are described in detail in Chapter 7.

It is noted that the health impact function can also be used to evaluate the public health burden of ambient levels of air pollution exposure. In this case, $\Delta C$ is evaluated with respect to a reference concentration of zero, yielding, the number of deaths or morbidities due to ambient exposure to a specific pollutant, referred to as the public health burden of air pollution.

In benefit-cost assessment, health impacts are often monetized for comparison with the costs of implementing air quality policies. For this comparison to be made, health "benefits" or "damages", depending on the perspective taken, are evaluated using monetary values from the economics literature based on the principles of willingness-topay or cost-of-illness, which differ among health outcomes. The monetized health impact function takes the form,

$$
\Delta M(\$)=M_{0} P\left(1-e^{-\beta \Delta C}\right) \cdot V_{S L}
$$

The difference between Equations 3.11 and 3.12, which are formulated for mortality, lies in the inclusion or exclusion of VSL for monetizing mortality counts. It is noted that the recommended VSL in the U.S. for use in Equation 3.12 is $\$ 7.9$ million per statistical life, in 2008 USD (U.S. EPA 2010). In Canada, the recommended VSL used in AQBAT is based on a discrete distribution with a central estimate of $\$ 7.17$ million in 2013 CAD (Chestnut et al. 2009). 


\subsubsection{Quantifying Health Impacts Using Adjoint Sensitivity Analysis}

Assessing the public health impacts of emissions and changes in emission behaviours using adjoint sensitivity analysis requires definition of a health-based adjoint cost function. Such an adjoint cost function might be defined as the number of premature mortalities in a given population that are attributable to air pollution exposure, as below,

$$
J=\sum_{t} \sum_{\omega} M_{0}(\omega) P(\omega) \cdot\left(1-e^{-\beta \Delta C(t, \omega)}\right)
$$

All variables in Equation 3.13 are as defined in Equation 3.11, with the difference that here, $M_{0}(\omega)$ and $P(\omega)$ are specific to each location, $\omega$, and $\Delta C(t, \omega)$ is specific to each location and time for adverse short-term outcomes. The adjoint cost function for mortality is integrated spatially and temporally to be the number of deaths in a defined geographic area (such as the U.S. or Canada) attributable to exposure to a specified air pollutant (such as ozone) over a specified time period (such as the ozone season).

The adjoint forcing term is defined as the derivative of the local cost function, for location $\omega$ and time $t$, with respect to concentration in the same location and time. For a cost function defined as the mortality burden due to air pollution as in Equation 3.13, the adjoint forcing term becomes,

$$
\varphi_{i}=\frac{\partial g(C, t, \omega)}{\partial C_{i}(t, \omega)}=M_{0}(\omega) P(\omega) \beta e^{-\beta \Delta C(t, \omega)}
$$

A linearized version of Equation 3.13 can also be derived using a Taylor Series Expansion. For a relatively small excess risk of exposure (i.e., a small value of $\beta$ such as those for mortality due to short-term ozone exposure) and any practical change in 
concentration, $\Delta C(t, \omega)$, this produces negligible error and simplifies the adjoint forcing term calculation and implementation,

$$
\varphi_{i}=\frac{\partial g(C, t, \omega)}{\partial C_{i}(t, \omega)} \simeq M_{0}(\omega) P(\omega) \beta
$$

Note that Equations 3.14-3.15 are expressed as an excess mortality count per unit concentration. Alternatively, if the monetary value of health outcomes are of interest, an additional term for the VSL must be included in Equations 3.13-3.15.

\subsubsection{Distinguishing Between Short- and Long-term Health Impacts in Adjoint}

\section{Sensitivity Analysis}

Epidemiological data from time-series studies can be incorporated into the health impact and adjoint cost functions (Equations 3.11-3.13) for estimating the short-term or acute health risks of emissions and associated air pollution exposure. If data from cohort studies is instead introduced into Equations 3.11-3.13, estimates of the long-term or chronic health risks of air pollution exposure are the focus. In Equation 3.13, the adjoint cost function is written for acute health effects that occur shortly after exposure (in this case, acute exposure mortality). In this case, daily mortality counts attributable to air pollution exposure are integrated over the model simulation period (integration over $t$ ). As such, $\Delta C$ in Equations 3.13-3.14 refers to a daily exposure metric or daily change in exposure (e.g., daily maximum 8-hr average or change in 1-hr maximum ozone). In contrast, for chronic health impacts that occur as a result of long durations of exposure, $\Delta C$ in Equations 3.13-3.14 refers to a longer-term average concentration used in epidemiological studies (e.g., summertime average ozone or annual average $\mathrm{NO}_{2}$ or 
$\left.\mathrm{PM}_{2.5}\right)$. In this case, the adjoint cost function would still be integrated over locations but not over time and a social discount rate is typically used if there is evidence of a cessation lag between exposure and time of death.

The distinction between short- and long-term public health impacts is explored further in each of the research chapters. Application of Equations 3.13-3.14 for estimating the acute public health impacts of ozone and $\mathrm{NO}_{2}$ exposure are explored in detail in Chapters 4-6. In Chapter 7, modified versions of Equations 3.13-3.14 are applied for estimating chronic health impacts. Detailed explanations of these adjoint cost functions and forcing terms are included in Chapters 4-7. 


\section{Chapter: Source Attribution of Health Benefits}

This chapter was published under the title "Source Attribution of Health Benefits from Air Pollution Abatement in Canada and the United States: An Adjoint Sensitivity Analysis"1 in Environmental Health Perspectives in 2013. The published manuscript is reproduced in full in this chapter with permission from Environmental Health Perspectives. It consists of original research for which Amanda Pappin is the main contributor $(80 \%)$.

\subsection{Introduction}

Acute and chronic exposure to ambient air pollution has been directly linked with adverse human health effects, resulting in substantial social and economic burdens worldwide. Several time-series and cohort studies conducted over the past few decades have examined the effects of particulate matter (PM) and gas-phase pollutants on short and long-term mortality and morbidity. In application, the results of such studies have been linked with air quality modeling to estimate the global burden of air pollution (Anenberg et al. 2010; Brauer et al. 2012), the health impacts of intercontinental pollutant transport (Anenberg et al. 2009), and evaluation of control measures (Tagaris et al. 2010; West et al. 2006).

$\mathrm{O}_{3}$ is one of the major photochemical oxidants in ambient air whose short-term health effects have been widely researched (e.g., Bell et al. 2005; Burnett et al. 1997;

\footnotetext{
${ }^{1}$ Pappin AJ, Hakami A. 2013. Source attribution of health benefits from air pollution abatement in Canada and the United States: An adjoint sensitivity analysis. Environ Health Perspect 121:572-579.
} 
Katsouyanni et al. 2009). Bell et al. (2004), in a multicity U.S. study, estimated that a 10 ppb increase in 24-hr average $\mathrm{O}_{3}$ concentration was associated with a $0.52 \%$ increase in all-cause mortality. Subsequently, Ito et al. (2005) conducted a meta-analysis of singlecity time series studies worldwide and found a slightly lower estimate of $0.39 \%$ for a 10 ppb change in 1-hr maximum $\mathrm{O}_{3}$ concentration. In a study of 12 major Canadian cities, Burnett et al. (2004) associated a $30.6 \mathrm{ppb}$ change in 2-day moving average $\mathrm{O}_{3}$ concentration with a $2.74 \%$ change in non-accidental mortality. More recent cohort studies on the long-term effects of $\mathrm{O}_{3}$ (e.g., Jerrett et al. 2009) suggest that chronic $\mathrm{O}_{3}$ exposure may have a stronger influence on mortality and the potential to afflict substantially larger societal costs.

Unlike $\mathrm{O}_{3}$, there is a lack of consensus concerning the association between $\mathrm{NO}_{2}$ and short-term mortality, due in part to scarcity of epidemiological evidence (e.g., Latza et al. 2009; Stieb et al. 2008). However, in a Canadian study, Burnett et al. (2004) found that a 22.4 ppb increase in 3-day moving average $\mathrm{NO}_{2}$ concentrations was associated with a $2.31 \%$ increase in non-accidental mortality. This association was further examined by Brook et al. (2007), who concluded that $\mathrm{NO}_{2}$ is the best single indicator of species in the ambient pollution mixture whose human health effects are not yet fully understood.

Quantification of health effects can be extended to emissions as part of benefitcost analyses with the use of chemical transport models (CTMs) that relate emission rates to ambient concentrations of pollutants (e.g., Anenberg et al. 2010; West et al. 2006). Model-based studies have traditionally used a scenario-based approach that aims to quantify health effects that would result if emissions from all sources were reduced uniformly or based on a prescribed scenario. Such studies are beneficial in assessing the 
spatiotemporal distribution of health benefits resulting from prescribed changes in model inputs, but cannot feasibly quantify distinctions between health benefits related to emission reductions from sources in different locations and times.

The quantified relationship between CTM-based model outputs and inputs is referred to as sensitivity analysis in the context of this work. Sensitivity information relates changes in emissions coming from sources (e.g., $\mathrm{NO}_{\mathrm{x}}$ emissions from motor vehicles or a power plant) to concentrations seen at receptors (e.g., health effects related to $\mathrm{O}_{3}$ exposure in a city) and thus estimates how much influence a source has on a receptor. These influences can be attributed to changes in emissions for a group of sources altogether (e.g., the transportation sector), as in previous studies, or instead to emissions coming from each source individually, yielding source-specific information. In a benefit-cost analysis framework, it is beneficial to know the marginal influences of emissions from different source locations on health effects. This kind of source-specific information can be achieved using adjoint or backward sensitivity analysis in CTMs. In this approach, influences on receptors are traced back to individual sources at all locations in preceding times (hence the term backward).

Here, we present a proof-of-concept study for integration of health benefit assessment models and epidemiological data with the adjoint of CTMs (the tool used to conduct adjoint sensitivity analysis) by forming a direct linkage between health effects at a national scale and emission sources at each location. We apply our methodology to estimate the response of national short-term mortality (valuated as an overall "health benefit") in Canada and the U.S. from short-term exposure to $\mathrm{O}_{3}$ (and $\mathrm{NO}_{2}$ in Canada) to emission reductions in each location across North America. 


\subsection{Methodology}

\subsubsection{Adjoint Sensitivity Analysis}

As mentioned before, adjoint sensitivity analysis, within the context of this work, refers to estimation of influences coming from emissions at individual source locations on short-term $\mathrm{O}_{3}$ mortality aggregated across all receptors. The difference between the adjoint approach and more conventional methods for sensitivity estimation is one of perspective, and lies in the direction in which sensitivity information evolves through the model in time and space. Conventional methods for sensitivity analysis track influences of a source, or a group of sources (e.g., all power plants), forward in time and space to all receptors (e.g., Canada and the U.S.), and are therefore referred to as "forward methods". One such approach is the brute-force method where emission inputs to CTMs are changed in the model to estimate the resultant distribution of concentrations across all receptor locations and times. With this method, it is a prohibitively costly undertaking to estimate influences of individual sources, as each source requires its emissions to be perturbed separately. A particular type of brute-force method, known as zero-out sensitivity analysis, requires emissions from a particular source be set to zero on the premise that removing a source will reveal its overall influence. In contrast, adjoint sensitivity analysis is a "backward method" that calculates influences of each source location on a single receptor or an ensemble of receptors. A single adjoint simulation provides sensitivities of a model output with respect to inputs across all locations and times (e.g., how $\mathrm{O}_{3}$-related mortality in Canada changes as a result of a change in emissions in any location) without requiring any perturbations to be made to model inputs themselves. 
Detailed explanation of adjoint sensitivity analysis in air quality modeling can be found elsewhere (Hakami et al. 2007; Henze et al. 2007; Sandu et al. 2005); here, we provide a descriptive overview. As mentioned before, the adjoint method can provide information about influences of location-specific sources on a function such as nationwide mortality that depends on concentrations across many receptor locations. This concentration-dependent function is commonly called the adjoint cost function. We define the adjoint cost function as the monetary value of mortality $(M)$ resulting from short-term exposure to $\mathrm{O}_{3}$ (and $\mathrm{NO}_{2}$ in Canada). We use epidemiological concentration response functions, population data, and recorded baseline mortality rates to establish a concentration-based adjoint cost function. Our adjoint sensitivity results, therefore, estimate influences from emissions in different source locations and for different species on nationwide mortality metrics.

Linkage between epidemiological models and adjoint calculations is established through appropriate definition of the adjoint cost function. A change in mortality valuation $(\Delta \mathrm{M})$ associated with a change in pollutant concentration $(\Delta \mathrm{C})$ is often given by

$$
\Delta M=M_{0} \cdot P \cdot V_{S L}\left(1-e^{-\beta \Delta C}\right)
$$

where $M_{0}$ is the baseline non-accidental mortality rate, $P$ is the population, $V_{S L}$ is the value of statistical life (VSL), and $\beta$ is the concentration response factor based on epidemiological models. VSL is the most common mortality valuation metric and is a measure of an individual's willingness-to-pay to reduce their probability of death (Alberini et al. 2006). Studies that have quantified the health benefits of air pollution 
reduction have often concluded that mortality reduction is the largest contributor (Hubbell et al. 2005).

Adjoint sensitivity calculations are driven by the adjoint forcing term $(\varphi)$ in the same fashion that concentrations are driven by emissions in CTMs. By this analogy, adjoint forcing terms can be regarded as "sources of influence" in the same way that emissions are sources for concentrations. The adjoint forcing term is the local, marginal influence of a change in concentration $(C)$ on the adjoint cost function $(\varphi=\partial M / \partial C \approx \Delta M / \Delta C)$. The linearized form of Equation 4.1 results in the approximation of the adjoint forcing term

$$
\varphi=\frac{\partial M}{\partial C} \approx \frac{\Delta M}{\Delta C} \approx M_{0} \cdot P \cdot V_{S L} \cdot \beta
$$

Note that as $\beta$ is often a small value, the resulting error from this linearization is negligible for all practical purposes. As Equation 4.2 suggests, adjoint forcing terms, acting as the sources of influence, increase with the size of population. If only mortality valuation due to $\mathrm{O}_{3}$ exposure is considered, the forcing term applied would only include a concentration response factor for $\mathrm{O}_{3}$, but since $\mathrm{O}_{3}$ is influenced by other species in various locations through atmospheric chemistry and transport, emissions of other species (e.g., $\mathrm{NO}_{\mathrm{x}}$ and VOCs) are linked to $\mathrm{O}_{3}$-related mortality in CTMs.

\subsubsection{Health Outcome Valuation}

Our estimation of mortality valuation for Canada is based on $\mathrm{O}_{3}$ and $\mathrm{NO}_{2}$ shortterm mortality. The required Canadian data for Equation 4.2 are extracted from the Air Quality Benefits Assessment Tool (AQBAT) developed by Health Canada (Judek et al. 
2006), which considers $\mathrm{O}_{3}$ (daily 1-hr maximum) and $\mathrm{NO}_{2}$ (24-hr average) to have $\beta$ values of $8.39 \times 10^{-4} \mathrm{ppb}^{-1}$ and $7.48 \times 10^{-4} \mathrm{ppb}^{-1}$, respectively, based on (though not identical to) Burnett et al. (2004). We include $\mathrm{NO}_{2}$ in our analysis for Canada based on the recommendation of Brook et al. (2007) and because of its inclusion in the Canadian Air Quality Health Index (AQHI) (Stieb et al. 2008).

For the U.S., we use results from different epidemiological studies, based in full or part on U.S. time-series data, to examine the importance of choice of metrics based on different averaging periods. Our default U.S. estimations are based on the widely used $\beta$ value of $5.2 \times 10^{-4} \mathrm{ppb}^{-1}$ for 24-hr average $\mathrm{O}_{3}$ from Bell et al. (2004), but we also consider a $\beta$-value of $3.9 \times 10^{-4} \mathrm{ppb}^{-1}$ for daily 1-hr maximum $\mathrm{O}_{3}$ from Ito et al. (2005) for comparison. Our adjoint cost function for the U.S. includes only $\mathrm{O}_{3}$ since no commonly accepted association between $\mathrm{NO}_{2}$ and short-term mortality is available for the U.S.

Mortality valuation estimates driven by Equation 4.2 are a function of population demographics. For Canada, we use 2007 total population and annual non-accidental baseline mortality rates (with no distinction by age category) for each of Canada's census divisions from AQBAT. For the U.S., total population and baseline mortality rates were obtained for each county from the Centers for Disease Control and Prevention (CDC). Non-accidental mortality rates were calculated from International Classification of Disease (ICD)-10 codes A-R as in Bell et al. (2004). We apply VSLs in 2011 equivalents (adjusted using the Consumer Price Index) of \$5.7M CAD in Canada (from AQBAT) and $\$ 8.1 \mathrm{M}$ USD in the U.S. (U.S. EPA 2010). When influences on two countries are compared or added, exchange rate parity is assumed. 
Through monetary valuation of mortality, we aim to establish a benefit-cost assessment framework for streamlined comparison between societal benefits and associated pollution abatement costs. We will refer to our mortality count valuation as "health benefits" hereafter for simplicity, while recognizing that our calculated values represent a societal willingness-to-pay to reduce the risk of premature death. Our health benefit estimations are overall conservative in that we are accounting for short-term mortality from gas-phase pollutants without including morbidity or long-term effects. Note that we refer to health benefit influences of marginal source emission reductions when using the term "source attribution".

\subsubsection{Health Benefit Estimation Case Study}

We use the U.S. EPA's Community Multiscale Air Quality (CMAQ) model (Byun and Schere 2006) and its adjoint for health benefit source attribution. Description and validation of the adjoint of CMAQ can be found in Hakami et al. (2007). The current adjoint model for CMAQ only includes gas-phase processes (chemistry and transport) of 72 active species. Our application of CMAQ is driven by meteorology from the Weather Research and Forecasting (WRF) model (Skamarock et al. 2005) and emissions calculated on a day-by-day, hour-by-hour basis using the Sparse Matrix Operator Kernel Emissions (SMOKE) model (UNC IE 2009). Emissions are projected to our simulation year from the 2005 National Emissions Inventory (NEI) for the U.S. and the 2006 National Pollutant Release Inventory (NPRI) for Canada. Our simulation is conducted over a continental domain with a horizontal grid resolution of 36-km (i.e., a matrix of 36by $36-\mathrm{km}$ grid cells), 34 vertical layers extending into the stratosphere, and for the summer of 2007. When compared to $\mathrm{O}_{3}$ observations, our simulations show a $16.5 \%$ 
mean fractional error (MFE) and $+5.5 \%$ mean fractional bias (MFB) (see Appendix B). Therefore, our exposure metrics are fairly accurate but slightly overestimated; however, this bias in concentrations is not expected to have a significant impact on source attribution results. Without capturing source influences on exposure to PM (the adjoint of CMAQ for PM is still in development) or other short/long-term effects, we regard our study as a proof-of-concept analysis.

\subsection{Results}

We estimate Canadian and U.S. health benefits from $\mathrm{NO}_{\mathrm{x}}$ and $\mathrm{VOC}$ emission reductions in each location or grid cell (i.e., each 36- by 36-km box) (Figure 4.1). Estimated health benefit influences are reported in $\$ 1000$ 's per day ( $\$ \mathrm{~K} / \mathrm{day}$ ) for a $10 \%$ change in emissions in all layers, and represent daily contributions to annual health benefits (i.e., baseline mortality is scaled to a daily rate). For example, in Figure 4.1A, a value of $\$ 100 \mathrm{~K} /$ day in a grid cell indicates that a $10 \%$ reduction in $\mathrm{NO}_{\mathrm{x}}$ emissions from that cell would benefit Canada by $\$ 100 \mathrm{~K} /$ day in reduced mortality nation-wide, whereas in Figure 4.1B, a value of $\$ 100 \mathrm{~K} /$ day in a grid cell indicates that a $10 \%$ reduction in $\mathrm{NO}_{\mathrm{x}}$ emissions from that cell would benefit the U.S. by $\$ 100 \mathrm{~K} /$ day in reduced mortality nation-wide. Note that the adjoint method provides source-specific information but lacks receptor specificity, and therefore, the distribution of benefits across receptors (i.e., mortality reductions according to geographic location) cannot be seen in these results. However, the model does provide a means to quantify national-level benefits resulting from both domestic emission reductions and reductions in emissions in the adjacent country. Health benefits are average daily influences over July 1, 2007 to September 30, 2007, and are reported in each country's respective currency. 
A

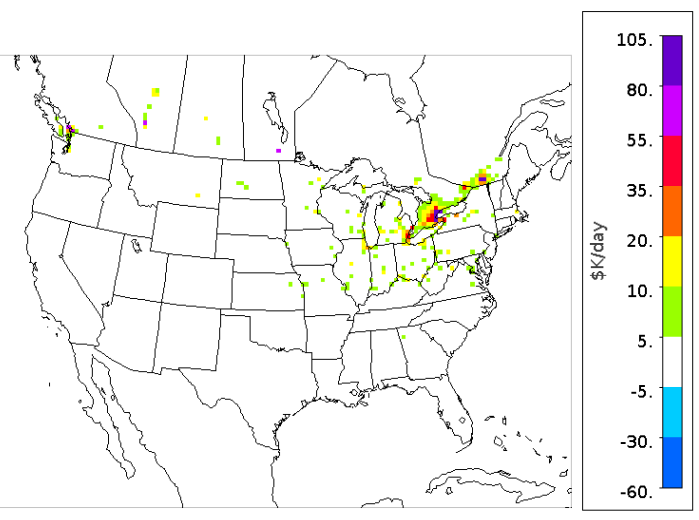

B
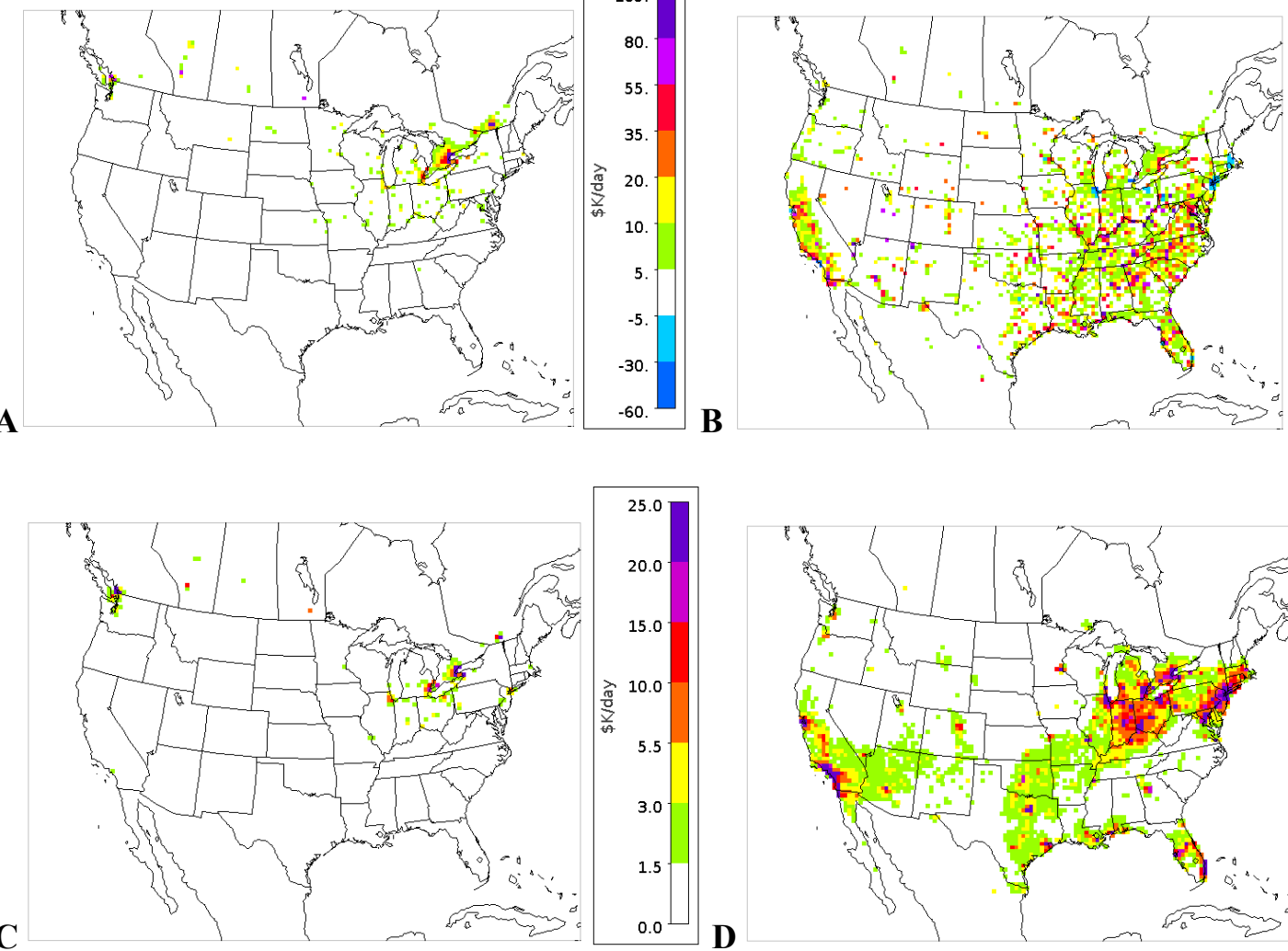

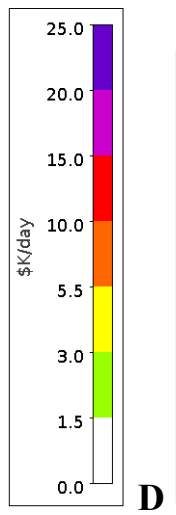

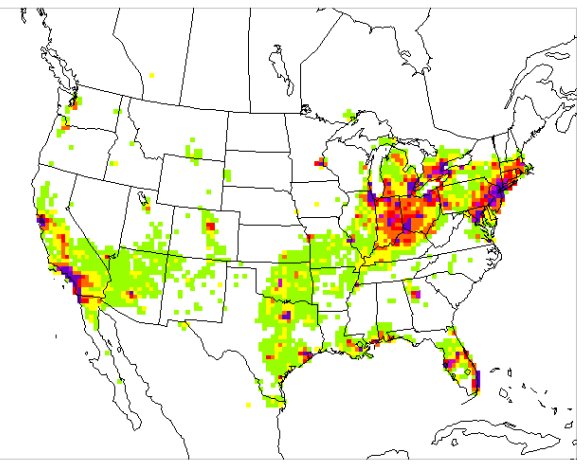

Figure 4.1 Average daily health benefit influences of emissions from individual source locations for Canada (left) and the U.S. (right) estimated for a $10 \%$ reduction in anthropogenic emissions of $\mathrm{NO}_{\mathrm{x}}$ (top) and VOCs (bottom). Health benefit influences on Canada account for both $\mathrm{O}_{3}$ - and $\mathrm{NO}_{2}$-related mortality (A,C), while influences for the U.S. account for mortality associated with $\mathrm{O}_{3}$ exposure only (B,D). Health benefits are average daily influences from July 1 to September 30, 2007. Note that benefits are shown according to the locations of the emissions sources that determine them, rather than the locations that experience the health benefits. For example, influences of both U.S. and Canadian $\mathrm{NO}_{\mathrm{x}}$ sources shown in panel $\mathrm{A}$ indicate nation-wide benefits experienced by Canadians only, whereas influences of U.S. and Canadian $\mathrm{NO}_{\mathrm{x}}$ sources shown in panel B indicate nation-wide benefits experienced by Americans only. 


\subsubsection{Attribution of Canadian Health Benefits to North American Sources}

Canadian health benefits from changes in exposure to both $\mathrm{O}_{3}$ (daily 1-hr maximum) and $\mathrm{NO}_{2}$ as a consequence of reductions in $\mathrm{NO}_{\mathrm{x}}$ emissions are shown in Figure 4.1A. First notable is the tendency of influences to exist in proximity to population centers in Canada, suggesting a strong local component to these health benefits. While emissions from sources in high-population urban areas will have a greater likelihood of influencing population exposure to $\mathrm{O}_{3}$ and $\mathrm{NO}_{2}$, their influence can be extended across the nations. Long-range influences of sources from locations in the U.S. on benefits accrued in Canada reflect the relatively long atmospheric lifetime of $\mathrm{O}_{3}$, while influences on $\mathrm{NO}_{2}$ occur more locally (for benefits related to $\mathrm{O}_{3}$ and $\mathrm{NO}_{2}$ separately, see Appendix B). The largest overall influence comes from emissions in Hamilton (upwind of Toronto), reaching \$253K/day (Figure 4.1A). Significant influences are also seen from emissions in the Windsor-Quebec Corridor and emissions from the north-eastern U.S. (e.g., $\$ 211 \mathrm{~K} /$ day in Montreal and \$47K/day in Detroit). VOC emissions have significantly lower estimated influences on mortality (Figure 4.1C), with the largest benefit seen for emission reductions upwind of the Greater Toronto Area ( $\$ 54 \mathrm{~K} /$ day for a $10 \%$ reduction) where a VOC-limited chemical regime exists on many summer days (i.e., production of $\mathrm{O}_{3}$ is more affected by VOC availability, rather than $\mathrm{NO}_{\mathrm{x}}$ concentrations). Canada-wide health benefits have consistently positive sensitivities to anthropogenic VOC emissions across the domain.

\subsubsection{Attribution of U.S. Health Benefits to North American Sources}

Health benefit influences on the U.S. from anthropogenic $\mathrm{NO}_{\mathrm{x}}$ emissions are calculated for $\mathrm{O}_{3}$ exposure only (based on a 24-hr average metric; Figure 4.1B). In 
comparison to results for Canada, contributions of North American $\mathrm{NO}_{\mathrm{x}}$ emissions towards U.S. mortality valuations are traced to sources dispersed over a wider geographic area and have generally higher magnitudes due to both larger populations and higher emissions in the U.S. The largest estimated benefits are from reductions in emissions from sources near Atlanta ( $\$ 181 \mathrm{~K} /$ day for a $10 \%$ reduction in $\mathrm{NO}_{\mathrm{x}}$ emissions); comparable to the influence seen from $\mathrm{NO}_{\mathrm{x}}$ emission reductions in Montreal in Figure 4.1A. We also estimate substantial negative sensitivities or disbenefits from emissions originating in large cities in the U.S. (e.g., New York and Los Angeles at -\$681K/day and $-\$ 244 \mathrm{~K} /$ day, respectively). These negative influences coincide with $\mathrm{NO}_{\mathrm{x}}$-inhibited atmospheric conditions where $\mathrm{O}_{3}$ production increases as $\mathrm{NO}_{\mathrm{x}}$ availability decreases, and thus reducing $\mathrm{NO}_{x}$ emissions increases $\mathrm{O}_{3}$-related mortality. This is in contrast with consistently positive benefits estimated for Canada (Figure 4.1A), where any disbenefits in $\mathrm{O}_{3}$-related mortality under $\mathrm{NO}_{\mathrm{x}}$-inhibited conditions are offset by concomitant benefits in $\mathrm{NO}_{2}$-related mortality. Our estimated benefits for the U.S. (Figure 4.1B) do not account for $\mathrm{NO}_{2}$ exposure and thus negative values persist under $\mathrm{NO}_{\mathrm{x}}$-inhibited conditions. We also observe that estimated benefits from reductions in VOC emissions (Figure 4.1D) are significantly higher in magnitude than for Canada, particularly for VOC-limited (or $\mathrm{NO}_{\mathrm{x}}$-inhibited) metropolitan regions (the largest influences are in $\mathrm{New}$ York and Los Angeles, at \$294K/day and \$272K/day for $10 \%$ reductions in VOC emissions in each city, respectively).

A few points about disbenefits from $\mathrm{NO}_{\mathrm{x}}$ emission reductions in large U.S. cities (Figure 4.2A) are worth mentioning. First, only $\mathrm{O}_{3}$-related mortality is included in our health benefit estimates. If PM-related health effects are considered as well, these 
disbenefits are expected to diminish due to reduced inorganic PM concentrations. Second, adjoint sensitivities provide a measure of individual source (or location) contributions that, if considered in isolation, should be regarded as local in nature. In reality, emission reductions are likely to be introduced within a larger regional and/or national context which may alter adjoint source influences, and in some cases may turn disbenefits into benefits. Previous forward sensitivity studies have shown that influences of $\mathrm{NO}_{x}$ emissions on $\mathrm{O}_{3}$ concentrations remain linear up to about a $30 \%$ change in domain-wide $\mathrm{NO}_{\mathrm{x}}$ emissions (Hakami et al. 2004). Consequently, adjoint sensitivity estimates may not be valid over changes in emissions that are large enough to affect the chemical regime of the atmosphere. Therefore, in presence of widespread and substantial changes in emissions, a multistep analysis of health benefits (i.e., multiple adjoint simulations for gradually altered emission baselines over time) is more appropriate. Estimation of adjoint sensitivities along the emission control trajectory would result in gradually diminishing disbenefits as changes in emissions become substantial enough to shift the predominant chemical regime in cities away from a $\mathrm{NO}_{\mathrm{x}}$-inhibited environment. Finally, the results shown in Figure 4.1 do not consider positive trans-boundary influences (e.g., the benefits of reduced $\mathrm{O}_{3}$ exposure in Europe as a result of reducing U.S. $\mathrm{NO}_{\mathrm{x}}$ emissions).

\subsubsection{Temporal Variability in Health Benefit Influences}

The dependence of atmospheric pollutant transformation and transport on meteorological conditions causes a great deal of day-to-day variability in health benefit attributions. Figure 4.2 depicts time-variant influences of $10 \%$ reductions in $\mathrm{NO}_{\mathrm{x}}$ or VOC emissions from sources in select cities on Canada-wide and U.S.-wide mortality due to $\mathrm{O}_{3}$ 
exposure. Daily snapshots (i.e., the spatial distribution of influences on specific days) are shown in Appendix B.

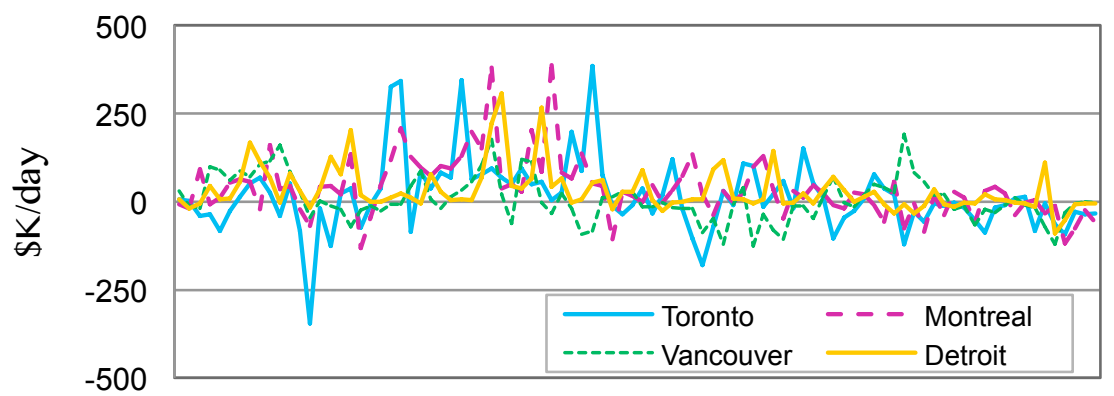

A

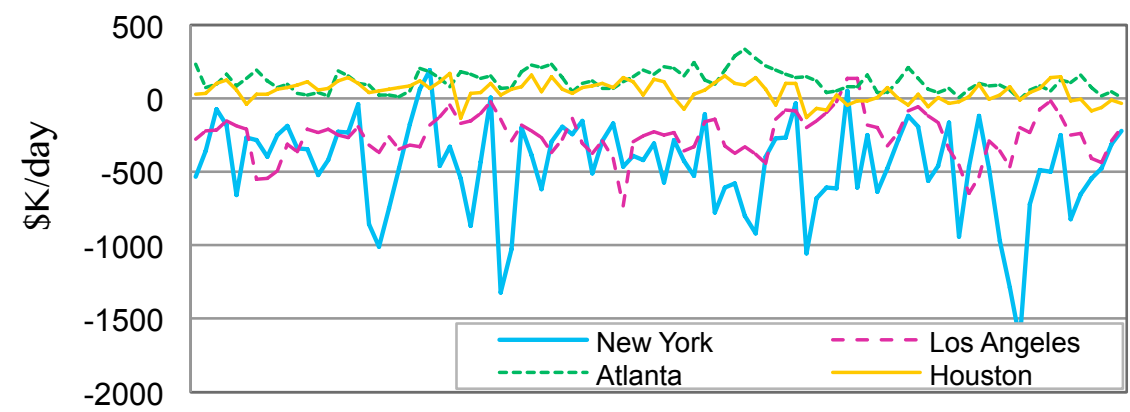

B

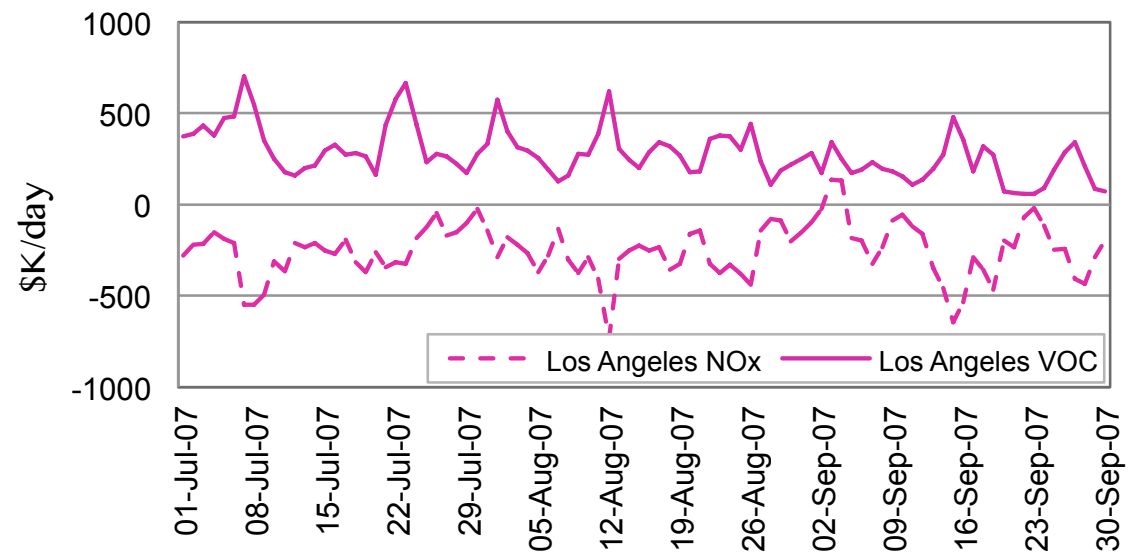

C

Figure 4.2 Daily variability of influences from a $10 \%$ reduction in anthropogenic emissions of $\mathrm{NO}_{\mathrm{x}}$ originating from major cities on short-term mortality due to $\mathrm{O}_{3}$ exposure in (A) Canada and (B) the U.S. Daily variability in $\mathrm{NO}_{\mathrm{x}}$ and VOC influences from Los Angeles on mortality in the U.S. is shown in (C). Influences are shown for single grid cells coinciding with the center of each city. 
Significant day-to-day fluctuations in health benefit influences are evident for emission sources in all cities. Reductions in emissions from sources in major Canadian cities (e.g., Toronto and Montreal; Figure 4.2A) result in some days with sizably negative influences on $\mathrm{O}_{3}$-related mortality in Canada (though increases in $\mathrm{O}_{3}$-related mortality are counteracted by decreases in $\mathrm{NO}_{2}$-related mortality that are not shown in Figure 4.2). In the case of the U.S., $\mathrm{NO}_{\mathrm{x}}$ emission reductions in New York and Los Angeles (Figure 4.2B) contribute, on average, large disbenefits to national $\mathrm{O}_{3}$ mortality. On the other hand, reductions in emissions from sources in or near Atlanta show consistent benefits on daily $\mathrm{O}_{3}$-related mortality due to the abundance of biogenic VOCs and a predominantly $\mathrm{NO}_{\mathrm{x}}$-limited chemical regime (such that $\mathrm{O}_{3}$ production is always expected to decrease as $\mathrm{NO}_{\mathrm{x}}$ emissions are reduced). Furthermore, strongly $\mathrm{NO}_{\mathrm{x}}$-inhibited urban cores such as Los Angeles exhibit an inversely correlated behavior between $\mathrm{NO}_{\mathrm{x}}$ and $\mathrm{VOC}$ influences on day-to-day mortality because reductions in $\mathrm{NO}_{x}$ will promote $\mathrm{O}_{3}$ production (and increase $\mathrm{O}_{3}$-related mortality) under these conditions, while reductions in VOCs on the same days will decrease $\mathrm{O}_{3}$ production and related mortality (Figure 4.2C).

The significant daily variability observed in health benefit influences has important policy implications. Air quality decisions are understandably made based on the overall or average estimated impact of pollution control options. However, long-term measures taken based on average conditions may be effective on some days and ineffective on others. Significant day-to-day variability in our estimates suggests that targeted short-term measures guided by health benefit influences may complement longterm strategic planning for air quality improvement. While air quality forecasting efforts have so far been focused on concentration predictions, forecasting health benefit 
sensitivities for guiding short-term emission modification seems to be the next logical step.

\subsection{Discussion}

Figure 4.1 provides basic aggregate influences on Canada and U.S. health benefits from various anthropogenic sources in North America. In the following sections, we explore and discuss policy consequences of these results in more detail.

\subsubsection{Cross-border Transport of Health Benefits}

To assess the impact of cross-border transport on national mortality, we sum health benefit influences coming from emission sources within Canada and the U.S separately for two scenarios: (1) Canadian populations as the receptor for $\mathrm{O}_{3}$ and $\mathrm{NO}_{2}$ exposure and (2) U.S populations as the receptor for $\mathrm{O}_{3}$ exposure. These summations should be regarded as marginal influences due to a modest decrease in emissions (i.e., $10 \%$ ) rather than total contributions (or apportionment) resulting from setting all emissions to zero and thus removing the total influence of each country. As before, we use VSL and epidemiological statistics consistent with the approaches taken and/or time series studies done in each country.

When Canadian populations are the receptor for $\mathrm{O}_{3}$ and $\mathrm{NO}_{2}$ exposure, almost all of the long-range influences from U.S. emissions are due to $\mathrm{O}_{3}$ exposure. If all $\mathrm{NO}_{\mathrm{x}}$ sources in the U.S. reduced emissions by $10 \%$, Canada would experience an average estimated benefit of $\$ 3.8 \mathrm{M} /$ day (less than one death per day at a VSL of $\$ 5.7 \mathrm{M}$ ). Similarly, a $10 \%$ reduction in all Canadian $\mathrm{NO}_{\mathrm{x}}$ emissions would produce an average benefit of $\$ 4.0 \mathrm{M} /$ day on Canadian health. When the U.S population is the receptor for $\mathrm{O}_{3}$ 
exposure, cross-border transport of $\mathrm{NO}_{\mathrm{x}}$ resulting from a $10 \%$ reduction in emissions from Canadian sources would result in an average benefit to the U.S. of $\$ 1.7 \mathrm{M} /$ day, while the total influence of a $10 \%$ reduction in U.S. emissions on American health benefits is estimated to be $\$ 51.5 \mathrm{M} /$ day (approximately 6 deaths per day at a VSL of $\$ 8.1 \mathrm{M}$ ). In comparison with $\mathrm{NO}_{\mathrm{x}}$, cross-border influences of VOC emissions on both Canadian and U.S. populations are substantially smaller and more local in nature

The absolute magnitudes of cross-border mortality influences are comparable for the U.S. and Canada. However, even in the case of Canadian health benefits, there is a significant domestic component. On specific days, cross-border transport of U.S. emissions may have a greater influence on Canadian mortality than domestic emissions (see examples in Appendix B), but in general, we estimate that significant benefits would be gained from domestic emission controls in Canada. Also, an examination of influences by emission release layers shows that surface emissions (layer 1) are by far more influential than elevated sources (layers 2-8) (see Appendix B). This suggests that transportation emissions may be more influential on $\mathrm{O}_{3}\left(\right.$ and $\left.\mathrm{NO}_{2}\right)$ mortality than industrial sources.

\subsubsection{Effect of Averaging Period on Health Benefit Influences}

In results presented so far we use daily 1-hr maximum $\mathrm{O}_{3}$ exposure metrics to estimate benefits for Canada, and 24-hr average $\mathrm{O}_{3}$ exposure metrics to estimate benefits for the U.S., as these are the common metrics used in each country. Daily average and 1hr maximum $\mathrm{O}_{3}$ concentrations are often correlated, but would respond differently to emission reductions of $\mathrm{O}_{3}$ precursors. To explore the impact of the choice of metric on health benefit estimates, we repeat our adjoint calculations for U.S. mortality based on a 
daily 1-hr maximum $\mathrm{O}_{3}$ concentration response factor from Ito et al. (2005). As the Ito study and Bell et al. (2004) use different underlying data, our comparison should be regarded as qualitative; we mainly aim to examine differences in patterns and tendencies.

Health benefit estimates based on the 1-hr exposure metric (Figure 4.3B) are consistently higher than estimates based on the 24-h average metric (Figure 4.3A). More importantly, some locations that exhibit negative sensitivities (i.e., where emission reductions are associated with increased mortality) with the 24-hr averaging period (e.g., around the Great Lakes) have sizable estimated benefits based on a 1-hr metric. This is expected as the daily exposure metric includes night-time influences when $\mathrm{NO}_{\mathrm{x}}$ reductions are likely to result in increased $\mathrm{O}_{3}$ concentrations, resulting in negative influences on mortality. In contrast, $\mathrm{NO}_{\mathrm{x}}$ reductions during the day are more likely to have beneficial influences due to reductions in $\mathrm{O}_{3}$, except in urban environments that are extremely $\mathrm{NO}_{\mathrm{x}}$-inhibited. In extremely $\mathrm{NO}_{\mathrm{x}}$-rich urban cores such as New York or Los Angeles, $\mathrm{NO}_{\mathrm{x}}$ disbenefits persist (or can become more significant) even with exposure metrics based on 1-hr maximum concentrations. Although we examine only 1-hr and 24hr metrics for the U.S., these diurnal tendencies are an important consideration for the 8$\mathrm{hr} \mathrm{O}_{3}$ metric used in regulations. 

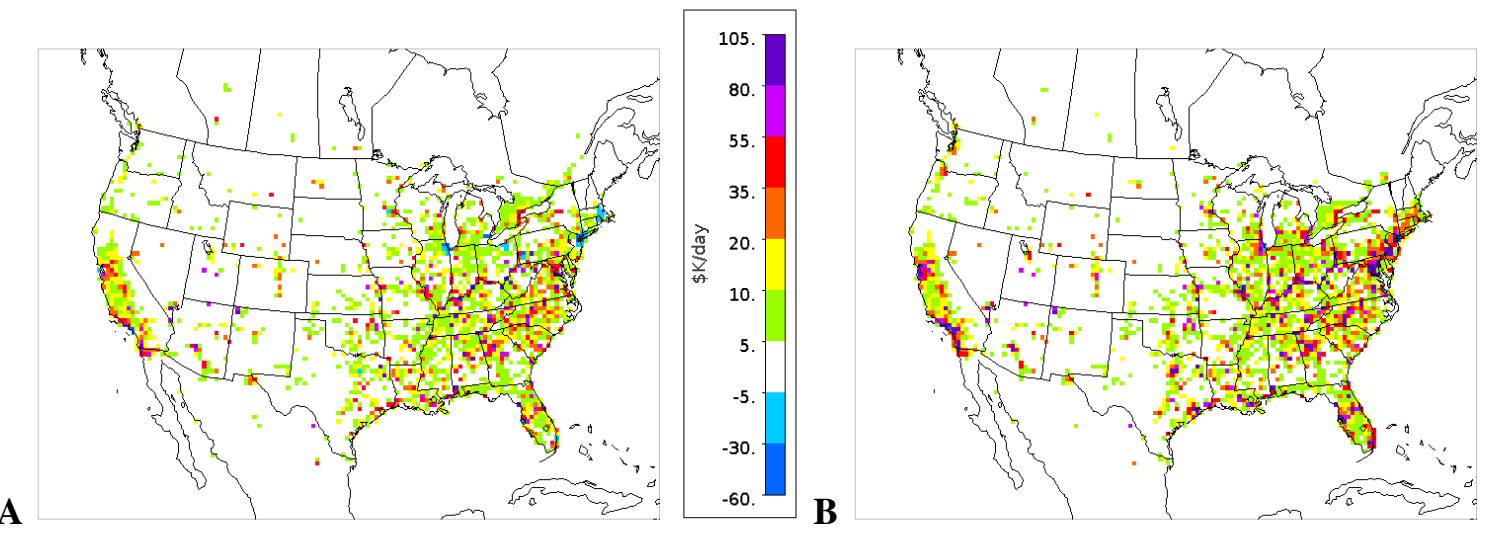

Figure 4.3 Average daily influences on U.S. short-term mortality estimated for various averaging periods from a $10 \%$ reduction in anthropogenic emissions of $\mathrm{NO}_{\mathrm{x}}$. Health benefit influences are calculated based on 24-hr average $\mathrm{O}_{3}$ concentrations (A, as in Figure 1B) and daily 1-hr maximum (B) $\mathrm{O}_{3}$ concentrations.

\subsubsection{Health Benefit Influences of Unit Source Reductions}

Day-to-day and temporal average health benefit influences are a function of (1) population demographics, (2) physical and chemical environmental processes that define source-receptor relationships, and (3) the magnitude of emissions at each source location. In general, emissions of $\mathrm{NO}_{\mathrm{x}}$ or $\mathrm{VOCs}$ from a grid cell will have a relatively large influence if they are large in magnitude, or if there is a large population exposed to their emissions, or both. A grid cell nearby a populous area has a large potential for influencing human health, but if that location has very little actual emissions, it will exert no influence on estimated benefits. To remove the inherent dependency of sensitivities on the spatial and temporal distribution of emission quantities, we estimate health benefit influences for hypothetical unit reductions in anthropogenic $\mathrm{NO}_{\mathrm{x}}$ and $\mathrm{VOC}$ emissions of 1 tonne/yr (Figures 4.4A-B). Unit reductions of $\mathrm{NO}_{\mathrm{x}}$ and $\mathrm{VOC}$ emissions are spread evenly throughout all days of the year and are based on domain-wide diurnal emission patterns assigned to all grid cells equally. The resulting influences represent marginal, 
annual benefits (extrapolated from summer months to the full year) from unit emission reductions at each location, which can be invaluable decision-making parameters. The results depict the overall influence of the same reduction in each source on both Canada $\left(\mathrm{O}_{3}\right.$ and $\left.\mathrm{NO}_{2}\right)$ and the U.S. $\left(\mathrm{O}_{3}\right)$.

These estimated health benefit influences have significantly greater spatial coverage than the estimates shown in Figure 4.1. Health benefit influences mainly reflect benefits from $\mathrm{NO}_{\mathrm{x}}$ reductions, and are highest along the Windsor-Quebec Corridor (Canada) and in California (U.S.), consistent with the fact that adjoint forcing is driven by downwind populations (largest values of approximately $\$ 75 \mathrm{~K} / \mathrm{yr}$ in Santa Barbara, Simi Valley, and west of Montreal, Dorval). As expected, large cities have lower attributed benefits (due to a VOC-limited chemical regime resulting in increased $\mathrm{O}_{3}$ concentrations and $\mathrm{O}_{3}$-related mortality with reductions in $\mathrm{NO}_{\mathrm{x}}$ ). Estimates in Canada are generally larger as they include influences on both $\mathrm{O}_{3}$ and $\mathrm{NO}_{2}$ exposure. 

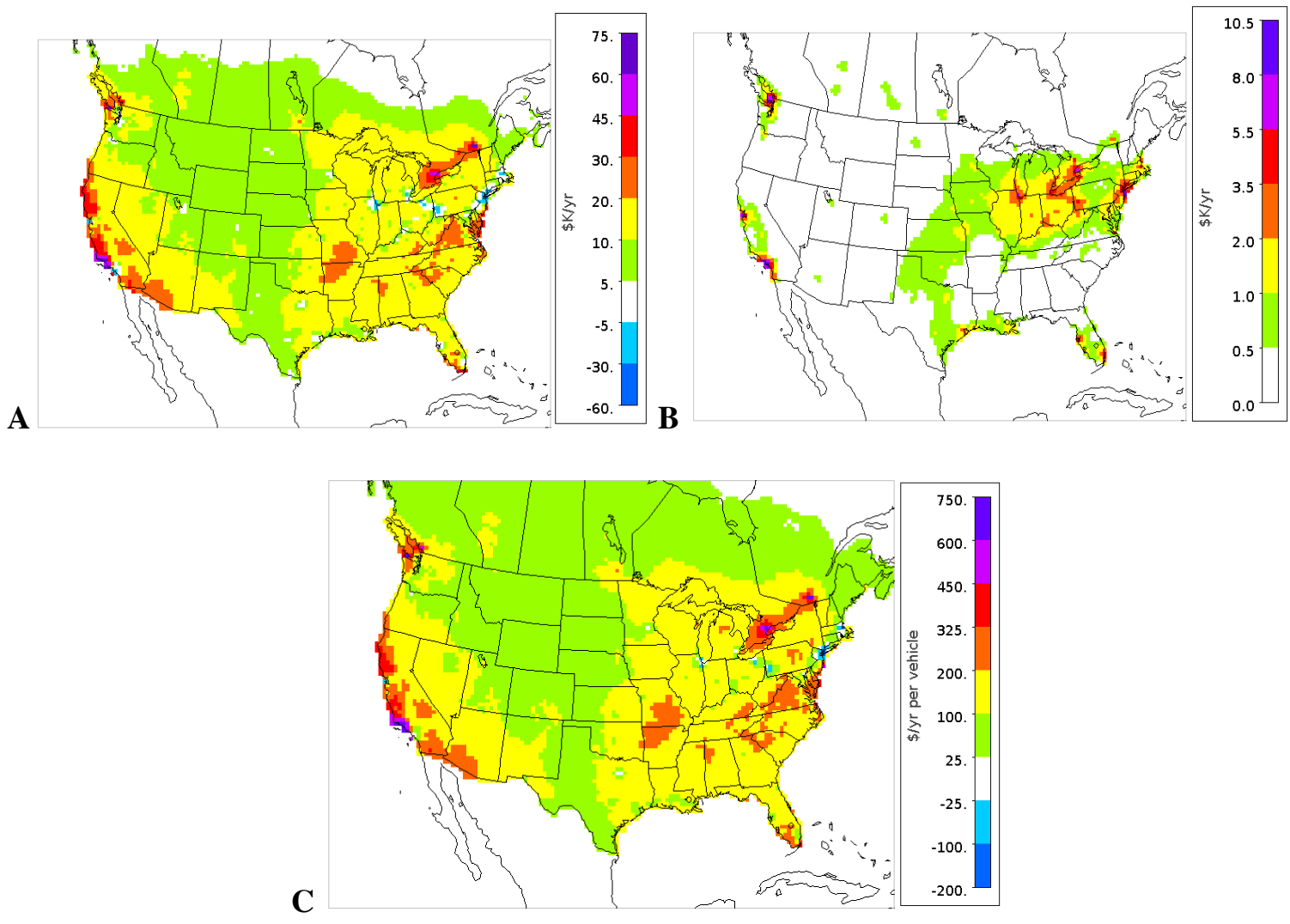

Figure 4.4 Average yearly influences of 1 tonne/yr reductions in anthropogenic surface-layer emissions of (A) $\mathrm{NO}_{\mathrm{x}}$ and (B) VOCs on North American short-term mortality. Unit reductions in emissions are distributed throughout all days and hours of the year based on domain-wide diurnal emission patterns assigned to each grid cell. Figure $4 \mathrm{C}$ shows estimated yearly benefits attributed to elimination of one average vehicle in a given location for both the U.S. $\left(\mathrm{O}_{3}\right)$ and $\mathrm{Canada}\left(\mathrm{O}_{3}\right.$ and $\left.\mathrm{NO}_{2}\right)$ combined.

Using fleet-average emission rates, values in Figure 4.4A and 4.4B can be translated into a benefit attribution map for personal vehicle use (Figure 4.4C). Values in Figure $4.4 \mathrm{C}$ can be interpreted as the yearly benefit of removing one average vehicle from the road in each grid cell, due to elimination of the vehicle's $\mathrm{NO}_{\mathrm{x}}$ and VOC emissions. Yearly benefits are calculated using annual per-vehicle emission rates of 0.010 $\mathrm{t} / \mathrm{yr}$ for $\mathrm{NO}_{\mathrm{x}}$ and $0.014 \mathrm{t} / \mathrm{yr}$ for VOCs as averages taken from the mobile emission 
inventory developed by SMOKE. Some major urban areas in the U.S. show small estimated influences from transportation (e.g., Los Angeles \$0/yr), with significant disbenefits estimated for New York (-\$750/yr), Boston (-\$150/yr), and a few other cities. In Canada, due to inclusion of $\mathrm{NO}_{2}$ in the adjoint cost function, no disbenefits are observed and estimated urban benefits are substantial, with the largest Canadian influences in Montreal (\$770/yr), Mississauga (\$440/yr), and Vancouver (\$450/yr). In the U.S., influences from the Pacific Ocean Highway in regions other than Los Angeles and the Bay Area are substantial, ranging between $\$ 300 / \mathrm{yr}$ and $\$ 830 / \mathrm{yr}$.

\subsection{Conclusions}

In this work, we use the adjoint of CMAQ to estimate nation-wide health benefits from reduced $\mathrm{O}_{3}$ - and $\mathrm{NO}_{2}$-related short-term mortality resulting from $\mathrm{NO}_{x}$ and $\mathrm{VOC}$ emission reductions in each source location. Our modeling period represents a single $\mathrm{O}_{3}$ season in 2007, and does not capture inter-annual variability in health benefit influences. Furthermore, while our calculations based on summer months are likely to overestimate annual benefits when extrapolated to the full year, we believe that, overall, we underestimate health benefits in not accounting for morbidity and long-term or PMrelated mortality.

Our estimates are affected by various uncertainties in epidemiological values, mortality valuation, emissions characterization, and atmospheric modeling (e.g., representation of complex atmospheric chemistry). Emission uncertainties are of particular importance as they are thought to be the major source of uncertainty in simulated concentrations (Russell and Dennis 2000). Sharp spatial gradients of health benefit influences can only be captured with higher resolution simulations (i.e., a smaller 
grid cell size). While these results provide insight into the general behavior of health benefit attributions, they should be regarded as a proof-of-concept demonstration of the adjoint method's capability to delineate health benefit influences. More conclusive quantification of influences requires further research with high-resolution, multi-year, multi-pollutant simulations that span over all possible health outcomes with adequate consideration for uncertainties.

Our results indicate important tendencies of health benefit influences:

1) From the day-to-day variability in health benefit influences, we infer that the efficacy of long-term pollution reduction measures could vary greatly in the shortterm.

2) We note a sizeable influence of cross-border transport, with the estimated influence of U.S. emissions on Canada being larger than the estimated influence of Canadian emissions on the U.S., but comparable in magnitude to the influence of domestic Canadian emissions on Canadian health. From a Canadian perspective, while the tendency to blame poor air quality on emissions in the U.S. seems somewhat justified, there is significant benefit to be gained from domestic emission controls.

3) Our results point to substantial differences in the response of exposure metrics to control of emissions when calculated for various averaging periods. These differences could have important regulatory implications and as such, this topic requires further investigation (with inclusion of the 8-hr metric) based on consistent underlying epidemiological models. 
4) Our estimates suggest that ground-level sources have the largest influences except where significant industrial activity exists. As such, we anticipate a potentially important application of this approach in transportation planning. For example, based on our results, we estimate health benefits of the subway system in Toronto to be approximately $\$ 130 \mathrm{M} / \mathrm{yr}$ from reduced short-term $\mathrm{O}_{3}$ and $\mathrm{NO}_{2}$-related mortality only.

5) Most importantly, our results suggest that potential health benefits are substantial and possibly under-represented in the current benefit-cost analysis frameworks that lack source specificity. For example, the U.S. market-based permit price (the average marginal abatement cost) available to power plants for 1 tonne of $\mathrm{NO}_{\mathrm{x}}$ emissions reduction during the $\mathrm{O}_{3}$ season in 2007 was approximately $\$ 900$ (U.S. EPA 2008). By contrast, our corresponding estimated $7^{\text {th }}$ layer (typical effective height for a power plant plume release) health benefit influence for the Ohio River Valley is approximately $\$ 11,000 / y r$. Such disparity between marginal abatement costs and marginal benefits can be best addressed using the sourcespecificity offered by the adjoint approach. 


\section{Chapter: Policy Analysis of Emission Control Benefits based on}

\section{Attainment and Exposure Metrics}

This chapter was published under the title "Attainment vs Exposure: Ozone Metric Responses to Source-Specific $\mathrm{NO}_{x}$ Controls Using Adjoint Sensitivity Analysis” in Environmental Science \& Technology in 2013. It is reprinted (adapted) with permission from Pappin AJ, Hakami A. 2013. Attainment vs exposure: Ozone metric responses to source-specific $\mathrm{NO}_{\mathrm{x}}$ controls using adjoint sensitivity analysis. Environ Sci Technol 47:13519-13527. Copyright 2013 American Chemical Society. It consists of original research for which Amanda Pappin is the main contributor (80\%).

\subsection{Introduction}

Regulatory standards and guidelines for ground-level ozone in North America have seen many changes over the past four decades due to progressions in the scientific understanding of pollution-related health effects (U.S. EPA 2013; U.S. EPA 2008). Various approaches to air quality management use different types of metrics to assess the severity of ambient ozone pollution. Such metrics might be based on thresholds above which ambient concentrations are deemed unacceptable (e.g., national standards), or instead, metrics quantifying adverse human health or environmental effects related to ozone exposure. In the past, air quality modeling studies have been conducted on the former threshold-based metrics in parallel with regulatory requirements for attaining standards (Cohan et al. 2006; Hakami et al. 2006), while a more recent focus on human health has developed (Anenberg et al. 2010; Berman et al. 2012; Cohan et al. 2006; Fang et al. 2013; Hubbell et al. 2005; Wong et al. 2003) in response to a growing body of 
evidence that adverse health effects occur at ozone concentrations below national standards (U.S. EPA 2013).

Current North American ozone standards specify that the annual fourth-highest daily maximum 8-hr average (DM8A; the largest summation of hourly ozone concentrations over a period of 8 consecutive hours, averaged, in a 24-hr period) concentration averaged over three consecutive years is not to exceed $0.075 \mathrm{ppm}$ in the U.S. and 65 ppb in Canada (CCME 2000; U.S. EPA 2008). By this definition, "nonattainment" for a region results when a representative 3-year average extreme concentration in a set of observations (termed a design value) is above the applicable standard for the nation.

Metrics such as mortality or morbidity aim to quantify the adverse health effects associated with ozone exposure of populations (referred to hereafter as exposure-based metrics) even at levels below national ozone standards. Exposure-based metrics assign a higher weighting to ozone concentrations found in more populous locations, and, in this way, urban and rural locations with the same degree of nonattainment are likely to have vastly different exposure-based metrics. While ozone standards are set based on the recommendations of collective risk assessments (U.S. EPA 2013) and thus indirectly account for population exposure, attainment-based metrics do not directly quantify the impacts of ground-level ozone on human health. More recent approaches in Canada have aimed to more directly introduce long-term exposure reduction targets into the development of standards (CCME 2007; CCME 2010). 
Science-based decision-making depends directly or indirectly on prioritizing emission control strategies by examining the responses of different metrics (attainmentor exposure-based and for different averaging periods, calculated over different locations or time periods; Bell et al. 2005) under realistic scenarios. Quantifying how various ozone metrics respond to changes in emissions behavior is appropriately addressed through sensitivity studies. Traditionally, the approach taken to conduct air pollution sensitivity analyses has been to use forward chemical transport models (CTMs) that yield information about locations that are most impacted by emissions behavior modification.

“Adjoint" or backward sensitivity analysis allows for differentiation between impacts and influences exerted by different sources, which is particularly relevant to devising emission control policies that preferentially target the most influential emission sources.

In this work, we aim to demonstrate how various ozone metrics respond to changes in location-specific emissions behavior across North America. We compare the sensitivities of a national (1) attainment-based metric (probability-weighted extreme concentrations, as defined later) and (2) mortality from short-term ozone exposure to emission source reductions of $\mathrm{NO}_{\mathrm{x}}$ across time and space.

\subsection{Methods}

\subsubsection{Adjoint Sensitivity Analysis}

Adjoint sensitivity analysis aims to estimate how emissions from specific source locations and times contribute to various ozone metrics. The adjoint method is complementary to traditional approaches of forward sensitivity analysis that quantify the response of concentrations at each receptor location to reductions in only a single emission source, or instead, a collection of sources. Forward sensitivities thus reveal 
locations most influenced by these sources, but are not able to differentiate between influences on a source-by-source basis. In the opposite sense, adjoint sensitivities quantify the size of influence each source exerts on a collective ozone metric defined across one or many receptors. The adjoint method thus gives source-specific information but lacks the receptor specificity offered by forward methods.

Within the context of air quality modeling, adjoint sensitivities are calculated using the adjoint of a CTM, run backward in time to trace influences on an outcome (e.g., a policy metric) at a defined receptor (or group of receptors) to all model inputs (i.e., emissions at all locations) at all times. An overview and formulation of the adjoint method's application in modeling the response of mortality metrics to emission reductions is provided in previous work (Pappin and Hakami 2013A), and a detailed description of adjoint sensitivity analysis can be found elsewhere (Elbern et al. 2000; Hakami et al. 2007; Henze et al. 2007; Sandu et al. 2005).

The critical step in adjoint sensitivity analysis applications is the definition of the adjoint cost function $(J)$. The adjoint cost function is a scalar concentration-based metric defined over one or many receptors and is the metric for which we seek sensitivity or influence information. This metric may pertain to concentrations of single or multiple species at one or numerous receptor locations and may or may not be integrated in time. We define $J$ as a function of ozone concentrations for two sets of model simulations: (1) attainment-based design values, and (2) short-term exposure mortality, with both metrics integrated over Canada and the U.S. for the duration of the simulation episode. 


\subsubsection{Attainment: Probabilistic Design Value Estimation}

Attainment is often modeled deterministically, as determined by the four highest modeled concentration-days for a given location. However, CTMs are likely to estimate temporally averaged ozone concentrations more accurately than concentrations on specific days in a simulation period, especially when those days are conducive to extreme pollution episodes (Hogrefe et al. 2001B).Calculating sensitivities of attainment metrics for these specific days thus poses a problem in that model results are subject to a higher degree of uncertainty. One probabilistic approach is to assign a probability of attainment that is extracted from the probability distribution of the fourth-highest concentration (rather than the distribution of all concentrations) (Hogrefe et al. 2001A). While this approach seems intuitive, the probability of attainment offers little value in influence modeling from an air quality management perspective. In highly polluted regions, distributions of the fourth-highest concentration are insensitive to marginal changes in concentrations (and by extension, emissions), producing negligible sensitivities and dictating a focus on borderline or marginal nonattainment regions while ignoring severely polluted regions.

We instead define a probabilistic attainment-based metric such that "attainment" is influenced by more days of the simulation period than only the four highest concentration days. We do so through the use of a probability-weighted extreme concentration, which we refer to as a "probabilistic design value" (PDV) that assigns weight to each day's DM8A ozone concentration proportional to the probability of that day having one of the four highest concentrations of the distribution for that location. In so doing, days with higher probabilities of having the four highest concentrations are 
more influential on the attainment-based metric. Note that we apply probabilities of a specific day being among the four highest concentrations in the season, rather than being solely the fourth highest. This is an important distinction with consequences for influence modeling as the probability of being among the four highest concentrations is a monotonic function of concentrations while the probability of being the fourth highest is not.

As formulated below, the adjoint cost function for attainment, $J_{a}$, is an accumulation (integration) of PDVs across all receptor locations within Canada and the U.S. whose local PDVs exceed a threshold of $65 \mathrm{ppb}$. Note that our use of an "attainment-based" metric differs from the regulatory definition in that, in reality, attainment is assessed by county- or regional-level design values, while we are restricted to a single collective, nation-wide measure with the adjoint method. We use a threshold of $65 \mathrm{ppb}$ as the attainment threshold in Canada and in anticipation of the proposed revision to the primary ozone standard in the U.S. to a value between 60-70 ppb (U.S. EPA 2010).

$$
J_{a}=\sum P D V_{k}=\sum_{k=1}^{N}\left(\frac{1}{p_{T_{k}}} \sum_{i=1}^{n} x_{i, k} p_{i, k}\right): P D V_{k}>65
$$

Here, $x_{i, k}$ is the simulated DM8A ozone concentration (ppb) for day $i$ in location $k, p_{i, k}$ is the probability that $x_{i, k}$ is among the four highest modeled concentrations in the ozone season for location $k$, and $p_{T_{k}}$ is the summation of all $p_{i, k}$ over $n$ simulation days. As shown above, PDVs are summed over all locations, $N$, whose values exceed $65 \mathrm{ppb}$ (see Appendix $\mathrm{C}$ for a map of these locations). 
To calculate $p_{i, k}$, we apply binomial sampling with a successful event defined as a randomly selected concentration from a location's ozone distribution exceeding the simulated concentration $x_{i, k}$ for the day of interest. The probability $p_{i, k}$ that a particular day's modeled ozone concentration is among the four highest concentrations in the ozone distribution for that location is then given by:

$$
p_{i, k}=\sum_{j=0}^{3}\left(\begin{array}{l}
n \\
j
\end{array}\right) p_{x}^{j}\left(1-p_{x}\right)^{n-j}
$$

where $p_{x}$ is the probability that a randomly selected ozone concentration exceeds that day's simulated concentration $\left(P\left(X_{k}>x_{i, k}\right)\right)$. Concentrations used in calculation of PDVs are simulated concentrations because modeling the responsiveness of PDVs to source reductions requires a physics-based linkage between concentrations and emissions within a CTM. The 5-month ozone season (May 1 - September 30, 2007) sample distribution of $x_{i}$ for each location is assumed to be normal and to adequately represent the population of concentrations in the ozone season (i.e., extreme ozone concentrations in the year). The validity of the normality assumption for $x_{i}$ has been tested for a set of simulated concentrations in this study. Distributions of $x_{i}$ and $p_{x}$ for each location are assumed to be constant, with mean and standard deviation unaffected by small changes in a single day's simulated concentration.

Differentiating $J_{a}$, above, with respect to $x_{i}$ yields adjoint forcing terms required for adjoint simulations (see Appendix C). Adjoint attainment-based forcing terms quantify how PDVs respond to changes in concentration on a particular location-day (in units of $\mathrm{ppb} / \mathrm{ppb}$ ); changes that are then linked to emissions through backward integration 
of the adjoint model. The magnitude of adjoint forcing terms depends on where $x_{i, k}$ falls on the ozone concentration distribution. In general, any reduction in $x_{i, k}$ in a distribution of $x_{i}$ for any particular location, no matter where on the distribution, will reduce the associated $p_{i, k}$ due to the fact that the lower the concentration, the lower the probability of being an extreme value. Any reduction in $p_{i, k}$ associated with a reduction in $x_{i, k}$ will proportionally shift more weight to other values of the distribution such that $p_{T_{k}}=\sum p_{i, k}$ remains unchanged in calculation of the PDV. Reducing an initially large value of $x_{i, k}$ from the upper tail-end of the distribution will thus lower the PDV by shifting more weight to other, substantially smaller values of $x_{i}$; producing an overall large, positive sensitivity. If instead $x_{i, k}$ falls lower on the distribution, its contribution to the PDV is minimal and remains so for small changes in concentration because, for all practical purposes, $p_{i, k}$ is negligible to start with. Adjoint forcing terms under these conditions may be negative but negligible.

\subsubsection{Exposure: Mortality Estimation}

In contrast to attainment, exposure-based health metrics lack thresholds and thus influences of emissions on mortality are not susceptible to the same episode selection uncertainties when conducting sensitivity analysis. We define our exposure-based adjoint cost function, $J_{m}$, as the premature mortality count related to short-term ozone exposure in Canada and the U.S.

$$
J_{m}=\sum_{k=1}^{N} \sum_{i=1}^{n} M_{0_{k}} P_{k}\left(1-e^{-\beta \cdot \Delta x_{i, k}}\right)
$$


Here, $M_{0_{k}}$ is the non-accidental baseline mortality rate or BMR (number of deaths per million population per year, scaled to a daily rate, day ${ }^{-1}$ ) for location $k, P_{k}$ is the allage population in millions for location $k, \beta$ is the concentration response factor (expressed as the fractional increase in BMR for a $1 \mathrm{ppb}$ increase in ozone concentration; $\mathrm{ppb}^{-1}$ ), and $\Delta x_{i, k}$ is the modeled 1-hr maximum, 8-hr maximum or 24-hr average ozone concentration for day $i$ and location $k$ (where $\Delta$ represents the change from a baseline of zero). For consistency, we apply the same $\beta$-values across Canada and the U.S. from the widely-used Bell et al. (2004) study, while recognizing that Canadian epidemiological studies correlating all-cause mortality with ozone concentrations have generally yielded higher values (Katsouyanni et al. 2009). For comparison with attainment metrics of the same 8-hr averaging period, we apply a $\beta$-value of $4.27 \times 10^{-4} \mathrm{ppb}^{-1}$ for DM8A ozone. To quantify the effect of averaging period on exposure sensitivities, we model the response of exposure-based metrics for averaging periods of 1 and 24 hours using $\beta$-values of 3.35 $\times 10^{-4} \mathrm{ppb}^{-1}$ and $5.20 \times 10^{-4} \mathrm{ppb}^{-1}$, respectively, from the same study (Bell et al. 2004).

Adjoint forcing terms are calculated by linearizing $J_{m}$ with respect to $x_{i}$ using a Taylor series expansion that produces negligible error for small changes in concentration. Formulation of the adjoint forcing term is shown below (Pappin and Hakami 2013A),

$$
\frac{\delta J_{m}}{\delta x_{i, k}}=\frac{M_{0_{k}} P_{k} \beta}{t_{f}-t_{0}}
$$

Exposure-based adjoint forcing terms are non-zero and injected as sources of influence over the period $t_{0} \rightarrow t_{f}$ (the start and end hours of the period of maximum ozone concentration) for any given day and for the averaging period of $\Delta x_{i, k}$. Adjoint 
forcing terms are averaged over the period from $t_{0}$ to $t_{f}$ (the start and end hours of the period of maximum ozone concentration). Adjoint forcing terms for the exposure-based metric are driven by populations and BMRs obtained for 2007 from Canada's Air Quality Benefits Assessment Tool (AQBAT) and from the Centers for Disease Control and Prevention (CDC) for the U.S.

\subsubsection{Case Study}

The Community Multiscale Air Quality (CMAQ) model simulates chemical and physical atmospheric processes to estimate concentrations of pollutants across space and time based on emission quantities and meteorological parameters. We run CMAQ in the forward mode to obtain ozone concentration distributions needed for calculation of adjoint forcing terms. Our model performance evaluation over the ozone season simulation period from May 1 to September 30, 2007 indicates an 18.0\% mean fractional error and a $+0.9 \%$ mean fractional bias of simulated values compared to observations. To calculate adjoint sensitivities, we run the adjoint of CMAQ (CMAQ-adjoint version 4.5.1) for gas-phase processes only (Hakami et al. 2007). We calculate adjoint sensitivities over a continental North American domain at a $36 \mathrm{~km}$ horizontal resolution with a vertical structure divided into 34 layers. CMAQ and its adjoint are driven by meteorological inputs from the Weather Research and Forecasting (WRF) model (Skamarock et al. 2005) for 2007. Emission inputs are projected for the year 2007 based on the 2006 National Pollutant Release Inventory (Canada) and the 2005 National Emissions Inventory (U.S.). 


\subsection{Results and Discussion}

Our adjoint sensitivities show the response of collective, nation-wide attainmentand exposure-based metrics to emission reductions from each source location (i.e., grid cell) across North America. While our results reveal magnitudes of influence exerted by various sources, locations where changes in PDVs or ozone-related mortality occur are not identified in the adjoint approach, as this is a question more appropriately addressed by forward sensitivity methods. In differentiating between the effectiveness of emission source controls on national metrics, we know only that these impacts occur within the boundaries of Canada and the U.S based on our geographic definition of the adjoint cost functions.

Our spatial plots show the major sources of influence on (1) cumulative PDVs > $65 \mathrm{ppb}$ and (2) mortality related to short-term ozone exposure, both within Canada and the U.S. over the same period. We scale our sensitivities to amount to the reduction in PDVs or ozone mortality arising from a seasonal (May-September 2007) 10\% reduction in anthropogenic $\mathrm{NO}_{\mathrm{x}}$ emissions from each grid cell. This scaling ensures that the inherent linearity assumption in adjoint sensitivities is valid, as previous studies suggest that ozone responses to $\mathrm{NO}_{\mathrm{x}}$ controls remain near-linear for up to a 30-50\% change in domain-wide emissions (Cohan et al. 2005; Hakami et al. 2003; Hakami et al. 2004). An attainment-based sensitivity equal to $1 \mathrm{ppb}$ for any particular grid cell indicates that a $10 \%$ reduction in emissions originating in that cell over the ozone season results in a collective $1 \mathrm{ppb}$ reduction in PDVs within Canada and the U.S. Likewise, an exposurebased sensitivity equal to 1 death/season for a grid cell (e.g., Figure 5.2B) indicates that the same $10 \%$ reduction in emissions in that cell reduces premature mortality related to 
short-term ozone exposure in Canada and the U.S. by 1 death over the ozone season. To facilitate more direct comparisons between sensitivities of each metric, we show a normalized version of Figure 5.1 in Appendix C.

\subsubsection{Metric Responses to Location-Specific Source Reductions}

Influences on the national attainment-based metric show significant spatial variability across Canada and the U.S. (Figure 5.1A). Reductions in anthropogenic $\mathrm{NO}_{\mathrm{x}}$ emissions from sources in California, the western U.S., and across the eastern U.S. exert the most widespread influence on cumulative PDVs. Canadian sources by comparison show relatively little overall influence on PDVs (with the exception of emission reductions in southeastern Ontario). Domain-wide, a 9,200 cumulative ppb reduction in PDVs is gained from a uniform $10 \%$ reduction in all anthropogenic $\mathrm{NO}_{\mathrm{x}}$ emissions in Canada and the U.S (equivalent to a 1.4 ppb reduction in the national-average PDV). The single largest influence originates from $\mathrm{NO}_{\mathrm{x}}$ source reductions in Los Angeles, amounting to an overall $72.3 \mathrm{ppb}$ cumulative reduction in PDVs downwind for a 10\% emission reduction. Similar influences of 65.5 and $64.4 \mathrm{ppb}$ are attributed to $10 \%$ reductions in $\mathrm{NO}_{\mathrm{x}}$ emissions in New Mexico and Atlanta, respectively. A few source locations (e.g., in the state of Minnesota) exert overall negative, but small influences. In such cases, ozone concentrations rise in the vicinity of an emission reduction, and although concentrations further downwind (and hence PDVs) may decline, they do so outside the geographic extent of the attainment cost function, i.e., over bodies of water or in areas with PDVs below the 65 ppb threshold. Such reductions in PDVs are not captured in the adjoint cost function, leading an overall negative sensitivity. 
Source influences on the exposure-based metric (Figure 5.1B) more closely follow the spread of population in Canada and the U.S. in comparison to Figure 5.1A. $\mathrm{NO}_{\mathrm{x}}$ sources in California and the eastern U.S. are significant contributors to nationwide premature mortality as they were to PDVs, while western states formerly shown to exert large influences on PDVs (Figure 5.1A) are much less influential. A uniform 10\% reduction across all anthropogenic $\mathrm{NO}_{\mathrm{x}}$ sources results in an aversion of 930 premature deaths from reduced short-term ozone exposure over the season in Canada and the U.S. The single largest influence originates from $\mathrm{NO}_{\mathrm{x}}$ source reductions in Atlanta, estimated to reduce nation-wide mortality by 4.0 deaths/season (scaled for a $10 \%$ reduction). In contrast to the almost consistently positive attainment-based sensitivities, mortality responses to emission reductions can be negative in large U.S. cities such as Los Angeles (an excess of 5.3 deaths/season for a 10\% reduction in emissions). Negative sensitivities in general coincide with metropolitan areas whose high $\mathrm{NO}_{\mathrm{x}}$ levels inhibit ozone production within the vicinity of the city, thus any reduction in available $\mathrm{NO}_{\mathrm{x}}$ will further increase local ozone concentrations. As the adjoint method is a linearization of a nonlinear system, these negative values cannot be accurately extrapolated along the response surface to changes in emissions that are large enough to shift the atmospheric chemical regime away from $\mathrm{NO}_{\mathrm{x}}$-inhibition. As more aggressive nation-wide and regional emission control policies take effect over time, re-quantification of adjoint-based sensitivities is required to assess the impact of a changing emissions baseline (Pappin and Hakami 2013A).

Rather than report relative influences (i.e., for a 10\% reduction), adjoint sensitivities might, in the absolute sense, be based on the same 1 tonne/season emitted in 
any location. Expressing adjoint sensitivities per unit of emission reduction removes the dependency of source influence estimates on emission quantities themselves, and is of particular relevance if seeking to estimate the returns of various policy measures (Pappin and Hakami 2013A). We thus express adjoint responses of both collective ozone metrics (i.e., cumulative PDVs $>65$ ppb or mortality from short-term ozone exposure within Canada and the U.S.) as the seasonal change in either metric as a result of a 1 tonne/season $\mathrm{NO}_{\mathrm{x}}$ reduction in any particular source location (Figure 5.1C-D). It should be noted again that the exact locations experiencing the metric reductions within each nation remain unknown in adjoint sensitivity analysis. The same 1 tonne of $\mathrm{NO}_{\mathrm{x}}$ emissions in each location is distributed across the ozone season and vertical model layers according to the actual temporal and vertical profile of $\mathrm{NO}_{\mathrm{x}}$ emissions from SMOKE.

Sensitivities of attainment- (Figure 5.1C) and exposure-based metrics (Figure 5.1D) to unit reductions in $\mathrm{NO}_{\mathrm{x}}$ emissions are similar across the domain and do not distinguish between places that have low or high emissions. As before, sources in the western U.S. exert the strongest influences on PDVs. Influences originating in Arizona, Nevada, New Mexico and Utah are more prominent once the effect of emission quantities is removed (i.e., in Figure 5.1C compared to Figure 5.1A). Meanwhile, mortality shows only a marginal response to emission reductions in the aforementioned states, and instead responds most strongly to unit emission reductions along the coast of and extending into central California. Unit emission sensitivities are highest north of Los Angeles (a 1 tonne reduction in $\mathrm{NO}_{\mathrm{x}}$ per ozone season yields a cumulative $0.23 \mathrm{ppb}$ reduction in PDVs 
nationally) and west of Los Angeles (with the same unit reduction of emissions averting $1.1 \times 10^{-2}$ deaths/season nation-wide).

A
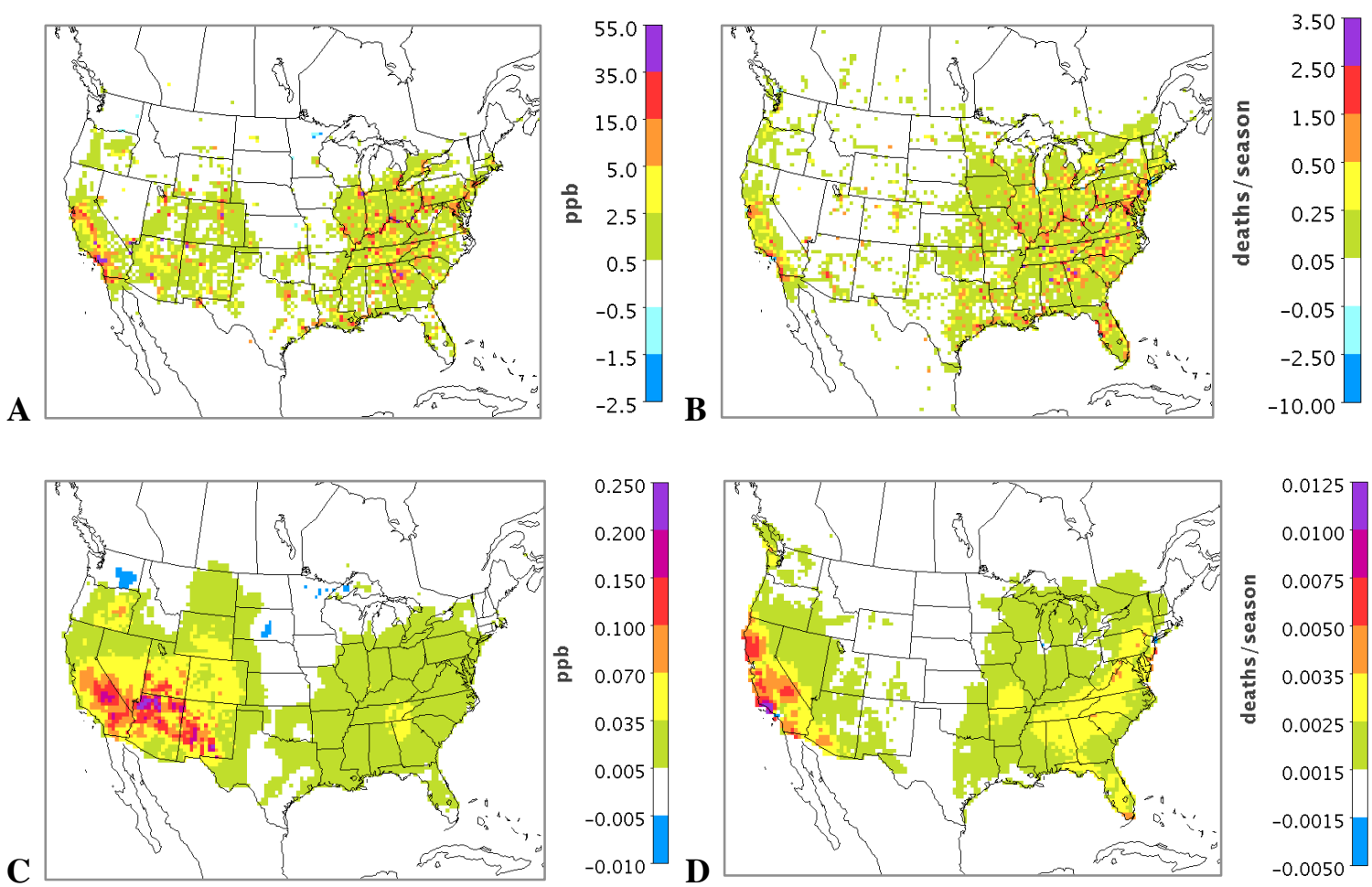

Figure 5.1 Influences of reductions in anthropogenic $\mathrm{NO}_{\mathrm{x}}$ emissions from each source location on the attainment-based metric (left panel; A, C) and exposure-based metric (right panel; B, D). Influences are scaled for a $10 \%$ reduction in $\mathrm{NO}_{\mathrm{x}}($ top; $\mathrm{A}-\mathrm{B})$ and a 1 tonne reduction in $\mathrm{NO}_{\mathrm{x}}$ (bottom; $\left.\mathrm{C}-\mathrm{D}\right)$ over the ozone season from each source location. Influences are integrated over May 1 -September 30, 2007.

\subsubsection{Metric Responses to Day-Specific Source Reductions}

The seasonal responses of ozone metrics to emission reductions shown thus far are temporal integrations of influences that vary considerably day-to-day under various emissions and meteorological conditions. We investigate this day-to-day variability of adjoint source influences by means of time-series plots for a sample of grid cells coinciding with major Canadian and U.S. cities (Figure 5.2). 
Responses of attainment- and exposure-based metrics to daily $\mathrm{NO}_{\mathrm{x}}$ emission controls in Los Angeles, Mississauga and Atlanta (Figure 5.2A-C, respectively) do not show any obvious correlation and highlight an important distinction between the two metrics. Construction of the attainment-based metric is such that different days carry different weights (or different influences on PDVs), whereas exposure-based metrics distinguish between different locations, by means of population weighting, but not different times. Influences on PDVs occur in clusters on only a fraction of simulated days when atmospheric conditions are conducive to high ozone concentrations. Such days coincide with days of a greater likelihood for extreme ozone concentrations at downwind receptors and carry significant weight (a high $p_{i, k}$ in Equation 5.1) in calculation of $J_{a}$. Non-influential days are the result of low values of $p_{i, k}$ among downwind receptor locations.

In comparison to attainment, exposure-based metrics exhibit more sustained fluctuations. Emission reductions on virtually all days contribute to ozone-related mortality because $J_{m}$ is equally influenced by the same change in ozone concentration, regardless of the baseline concentration to which the change is applied. As shown in past work (Pappin and Hakami 2013A), predictive short-term control strategies that consider day-to-day variability of source influences might complement long-term air quality management plans that are based on average, long-term responses. 
A

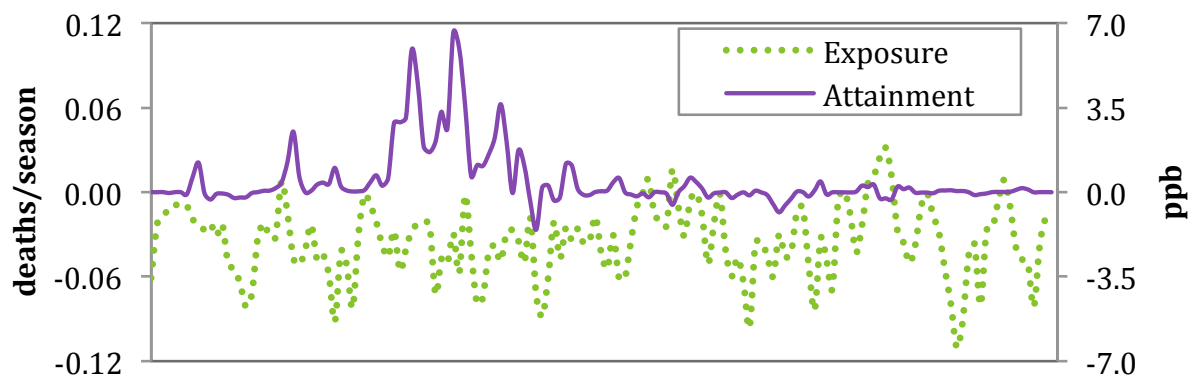

B
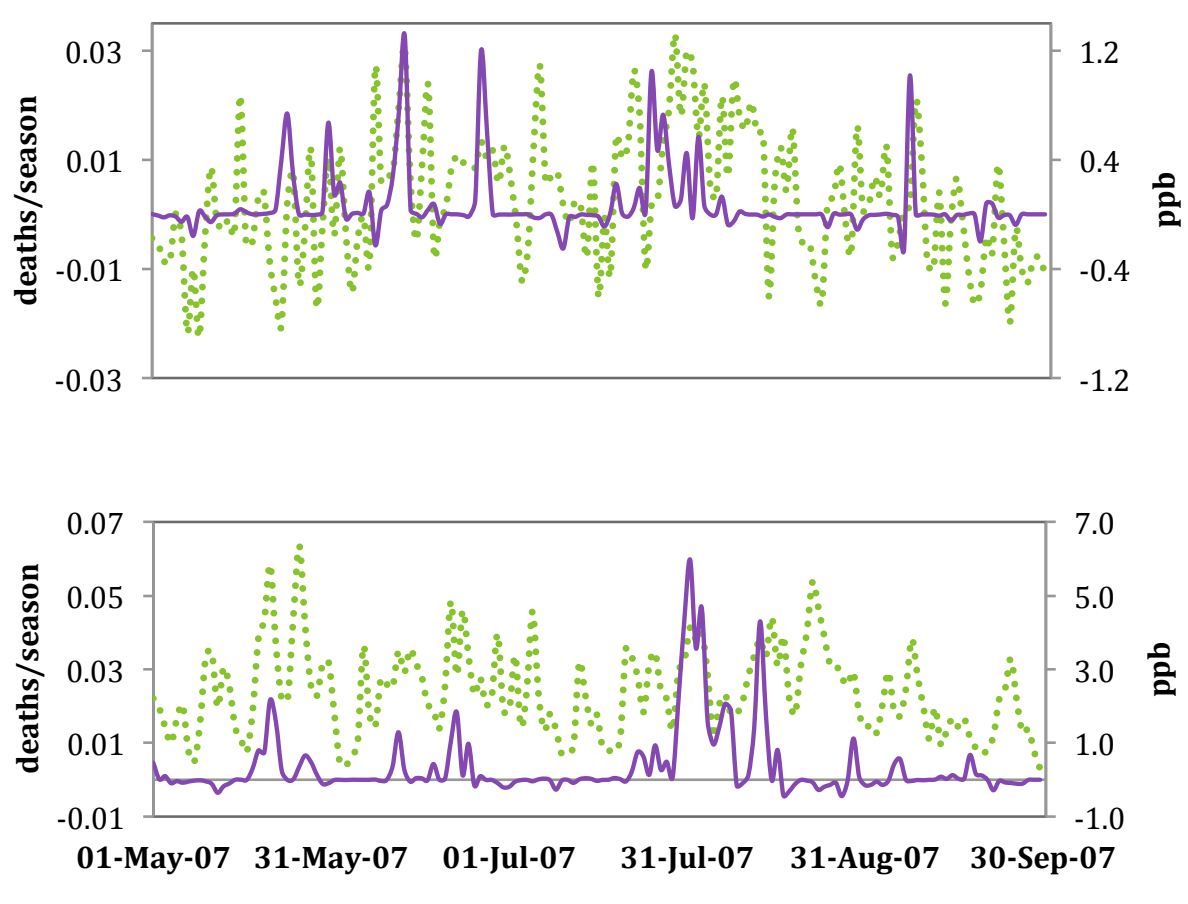

Figure 5.2 Daily influences of a $10 \%$ reduction in anthropogenic $\mathrm{NO}_{\mathrm{x}}$ from (A) Los Angeles, (B) Mississauga, and (C) Atlanta on the nation-wide attainment- based metric (solid line; purple) and exposure-based metric (broken line; green). Source locations refer to single 36 by $36 \mathrm{~km}$ grid cells coinciding with major cities.

\subsubsection{Variation of Exposure-Based Metric Responses with Averaging Period}

Responses of exposure-based metrics to emission reductions depend on the averaging period and the corresponding concentration response factor $(\beta)$ used for modeling health impacts. Up to this point, we compare attainment- and exposure-based 
metrics for an 8-hr averaging period as defined by current ozone standards (CCME 2000; U.S. EPA 2008). However, current health benefit assessment models quantify ozonerelated health effects for 1-hr (e.g., AQBAT in Canada, based on Canadian epidemiological studies) (Burnett et al. 2004) or 24-hr averaging periods (the Benefits Mapping and Analysis Program or BenMAP in the U.S., based on American epidemiological studies; Bell et al. 2004). For this reason, an examination of the consistency among responses of exposure-based metrics based on various averaging periods is pertinent. Thus, for completeness and as an extension of previous work (Pappin and Hakami 2013A), we highlight differences among metric responsiveness for pairs of 1-hr, 8-hr, and 24-hr averaging periods, subtracting sensitivities for the longer averaging period from the shorter period metric (Figure 5.3). Locations denoted in warm colors in Figure 5.3 indicate that the shorter averaging period metric is more responsive to a reduction in $\mathrm{NO}_{\mathrm{x}}$ emissions in that grid cell, while locations in blue suggest the opposite. Maps of exposure-based sensitivities for 1, 8 , and $24 \mathrm{~h}$ averaging periods shown to the same scale can be found in Appendix C.

With the exception of major urban areas, the 8-hr exposure-based metric exhibits more responsiveness to $\mathrm{NO}_{\mathrm{x}}$ emission reductions than both the 1-hr (Figure 5.3A) and 24hr metrics (Figure 5.3B). Urban centers of the U.S. (such as New York and Los Angeles) and Canada have largest sensitivities for the 1-hr metric compared with others (Figure 5.3A,C), such that overall, negative influences in such environments occur more frequently and vigorously with the 24-hr metric (Appendix C). The tendency of metrics based on longer time periods to show more negative responses to $\mathrm{NO}_{\mathrm{x}}$ emission reductions in urban locations is a result of diurnal changes in $\mathrm{NO}_{\mathrm{x}}$-radical interactions in 
the ozone production cycle. Negative sensitivities of $\mathrm{O}_{3}$ to $\mathrm{NO}_{\mathrm{x}}$ are commonplace during the nighttime and appear more prominently in exposure-based metrics for averaging times overlapping with periods of darkness (e.g., 24-hr and possibly 8-hr metrics).

In air pollution epidemiologic studies, a scaling to convert between concentration response factors ( $\beta$-values) over various averaging periods is often considered a useful, but simplified approximation (i.e., $\beta_{1 h r}=\beta_{8 h r} \times C_{1 h r} / C_{8 h r}=\beta_{24 h r} \times C_{1 h r} / C_{24 h r}$ ) (Schwartz 1997; Thurston et al. 2001). While the premise of this approximation may be valid in epidemiologic studies, such a transformation across exposure-based metrics of different averaging times can be flawed when linking metrics such as mortality to emissions in CTMs. This is due to the fact that diurnal changes in atmospheric processes render a simplified linear scaling between responses to emission controls fundamentally more problematic than in epidemiologic settings. Our findings indicate exposure-based metrics of an 8-hr averaging period as most responsive to emission reductions, illustrating a need for consistent and unified use of averaging periods in both epidemiological studies and policy development. 

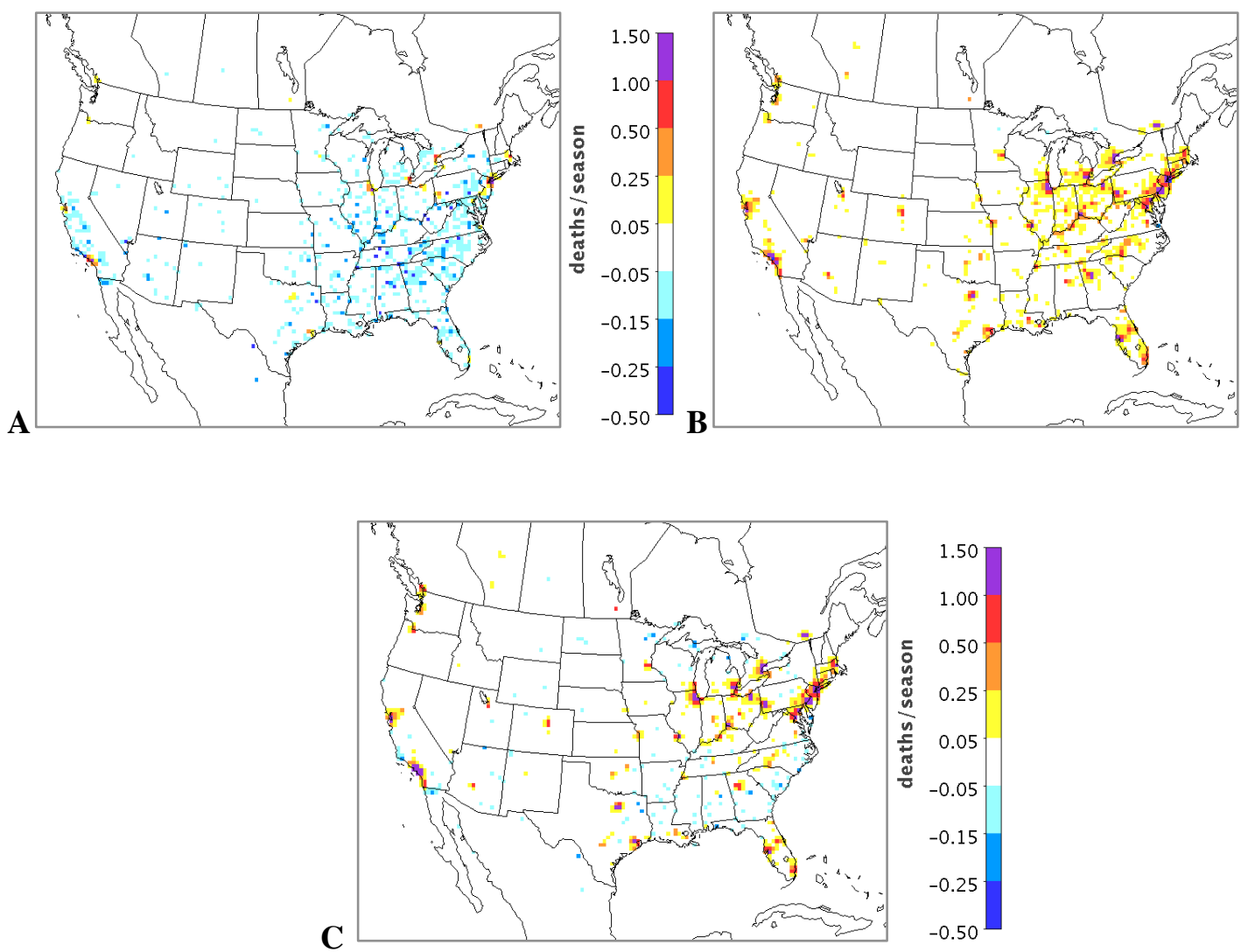

Figure 5.3 Differences between influences on exposure-based metrics for various averaging periods. Sensitivities are the responses of short-term exposure mortality to emission reductions in each source location, expressed as the difference between (A) daily 1-hr maximum and DM8A influences, or $\left(J_{m}(1 h r)-J_{m}(8 h r)\right),(B)$ DM8A and 24-hr average influences, or $\left(J_{m}(8 h r)-J_{m}(24 h r)\right)$, and (C) daily 1-hr maximum and 24-hr average influences, or $\left(J_{m}(1 h r)-J_{m}(24 h r)\right)$, all scaled for a $10 \%$ change in emissions of $\mathrm{NO}_{\mathrm{x}}$ in location $\boldsymbol{k}$. Influences are integrated over May 1 - September 30, 2007.

\subsubsection{Policy Considerations of Coupled Attainment and Exposure-Based Metric}

\section{Responses}

For clarity on the combined effectiveness of control measures on nation-wide attainment and exposure, we pair attainment- and exposure-based sensitivities for each location in the domain, and separate the coupled responses into effectiveness categories according to the magnitudes of influence exerted (Figure 5.4). We use thresholds for 
classifying sources whose controls are highly effective, with a high responsiveness to emission controls being sensitivities that are within the top $15 \%$ of those shown for the respective ozone metric in Figures 5.1A-B. The most effective controls from an attainment perspective (AttH category) have sensitivities greater than $1.25 \mathrm{ppb}$ for a $10 \%$ reduction in emissions, and similarly, we classify sensitivities whose absolute values exceed 0.15 deaths/season (with negative exposure-based sensitivities included) as most effective for reducing the exposure-based metric $(\mathrm{ExpH})$. Sensitivities smaller in magnitude than the $15 \%$ threshold indicate a low responsiveness for either metric (AttL or ExpL). We divide coupled responses into four categories where reducing $\mathrm{NO}_{\mathrm{x}}$ emissions significantly reduces (1) both metrics (AttH-ExpH), (2) exposure only (AttL$\mathrm{ExpH}),(3)$ attainment only (AttH-ExpL), or (4) attainment while significantly increasing exposure (i.e., a negative sensitivity; AttH-ExpHNeg). We show these regions on a spatial map (Figure 5.4A) and on a scatter plot of attainment- and exposure-based metric responses paired by location (Figure 5.4B).

From a policy point of view, control of $\mathrm{NO}_{\mathrm{x}}$ sources in most of California and the eastern U.S. (AttH-ExpH) is the most effective strategy for achieving tandem reductions in nationwide attainment- and exposure-based metrics (Figure 5.4A). A 10\% reduction in all sources falling within this effectiveness category yields an estimated 6,500 ppb cumulative reduction in PDVs and 613 less deaths/season. Sources clustered around population centers in the west and eastern U.S., as well as southeastern Ontario through Quebec, tend to be effective at reducing ozone-related health effects without having the same magnitude of influence on PDVs (AttL-ExpH). Contrarily, the western U.S. source region has sizable influence on PDVs but not mortality and falls into the attainment 
effectiveness category (AttH-ExpL). Finally, urban areas such as New York and Los Angeles exhibit negative exposure-based sensitivities (Att-H/Exp-HNeg) - emission controls here are counterproductive with regards to reducing ozone-related mortality but yield significant reductions in PDVs downwind in low-population areas that carry less influence in exposure-based metric calculations. The negative exposure effectiveness category is an extreme case where there is no clear relationship between attainment- and exposure-based metric responses. Within each of these broad categories of coupled metric responses, the degree of $\mathrm{NO}_{\mathrm{x}}$ reduction effectiveness spans over a wide range and can be seen in Figure 5.4B. 
A
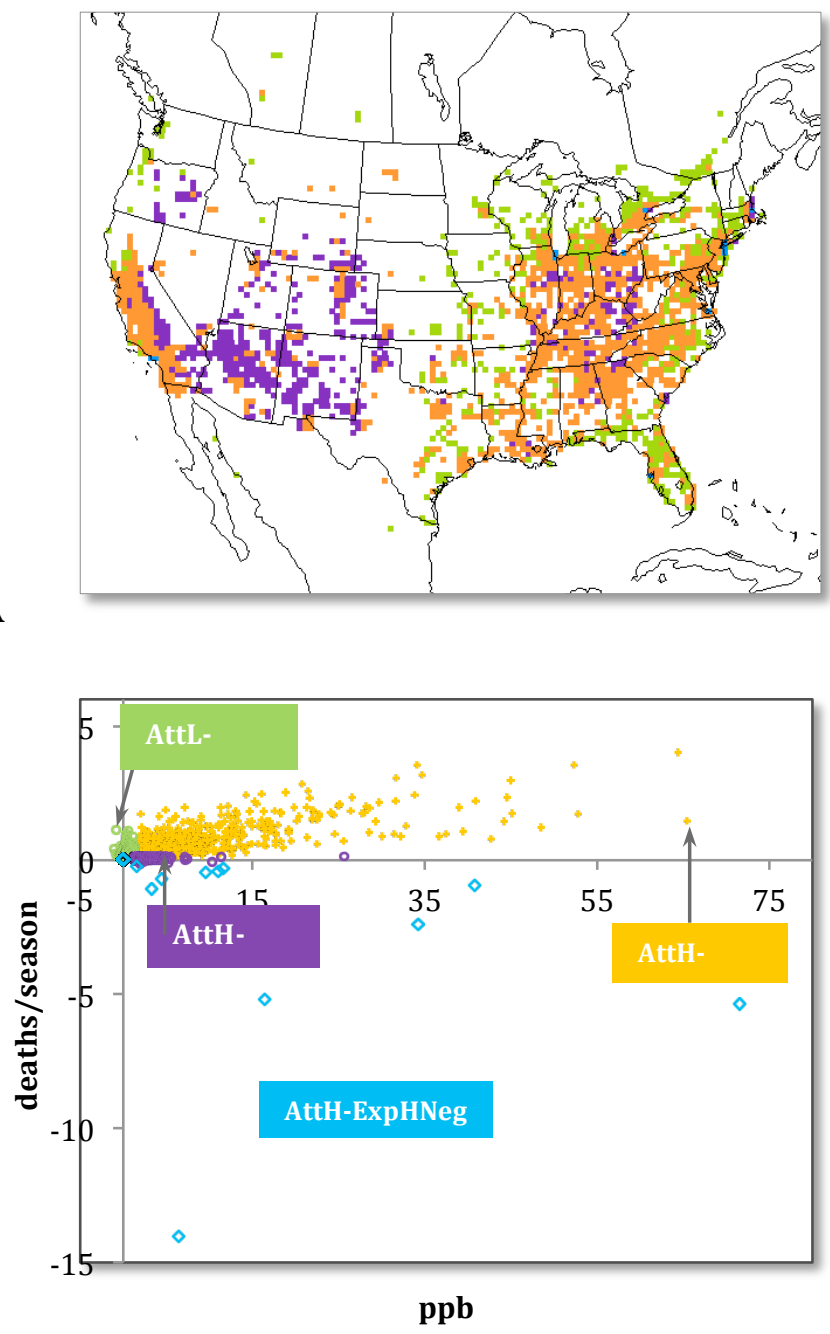

B

Figure 5.4 Effectiveness categories of coupled metric responsiveness to emission controls. (A) Source locations are categorized as having $\mathrm{NO}_{\mathrm{x}}$ emission reductions that most effectively reduce (1) both attainment and exposure-based metrics (AttH-ExpH; orange); (2) exposure only (AttL-ExpH; green); (3) attainment only (AttH-ExpL; purple); or (4) attainment, but with an increase in exposure (AttH-ExpHNeg; blue). (B) Each point represents a pair of attainment- and exposure-based sensitivities to a $\mathbf{1 0} \% \mathrm{NO}_{\mathrm{x}}$ emission reduction estimated for one source location.

The approach presented here is subject to a number of limitations. Our estimates of ozone metric responses to emission controls are affected by uncertainties inherent in epidemiologic models and in the representation of complex physical phenomenon and 
emission patterns in atmospheric modeling. Our estimations of attainment-based metric responses depend strictly on our probabilistic definition of the design value and cannot be accurately extrapolated into a regulatory context. True responses of attainment, as specified in the regulatory definition, are likely to differ from those reported here. Higher resolution and longer simulation timespans - preferably reaching up to three years - are desired for attainment response modeling. Finally, although the adjoint method reveals source-by-source influences, information is lost with regards to the distribution of impacts across receptor locations. Our results are thus best considered in tandem with complementary modeling approaches that reveal the distribution of attainment or health impacts across space and time.

Despite these limitations, the heterogeneity in attainment- and exposure-based metric responsiveness we illustrate suggests that strategies aiming to comply with regulatory standards (placing emphasis on ozone in extremely polluted regions without consideration for proximity to population centers) would potentially dictate a focus on emission sources whose reductions are non-influential on human health. Development of a regulatory paradigm for more direct inclusion of population exposure metrics in air quality management and decision making would provide a more effective means for reducing exposure and protecting public health. 


\section{Chapter: Diminishing Returns or Compounding Benefits of Air}

\section{Pollution Control?}

This chapter was published under the title "Diminishing Returns or Compounding Benefits of Air Pollution Control? The Case of $\mathrm{NO}_{\mathrm{x}}$ and Ozone" in Environmental Science \& Technology in 2015. It is reprinted (adapted) with permission from Pappin AJ, Mesbah SM, Hakami A, Schott S. 2015. Diminishing returns or compounding benefits of air pollution control? The case of $\mathrm{NO}_{\mathrm{x}}$ and ozone. Environ Sci Technol 49:9548-9556. Copyright 2015 American Chemical Society. This chapter consists of original research for which Amanda Pappin is the main contributor (60\%).

A comment on this paper appeared in Environmental Science \& Technology (Mendelsohn and Muller 2015). A version of the published response to the comment is included in Appendix D.

It is noted that during the defence of this thesis, clarification was sought regarding the nature of the emission reduction scenarios applied in this chapter. Each abatement scenario employed blanket or fixed-percentage reductions in all species emitted from anthropogenic source sectors (i.e., mobile and point sources). As such, emissions of $\mathrm{NO}_{\mathrm{x}}$ and VOCs, both precursors of ground-level ozone, were controlled with the same level of stringency. If $\mathrm{NO}_{\mathrm{x}}$ was the only species controlled, a higher ratio of $\mathrm{VOC}: \mathrm{NO}_{\mathrm{x}}$ would result in even larger marginal benefits than those reported in this chapter. A more detailed discussion of and justification for this approach can be found in Appendix D. 


\subsection{Introduction}

Estimating the health and environmental impacts of anthropogenic emissions is an important element of air quality decision-making. One measure of such impacts is marginal benefit $(\mathrm{MB})$, or the incremental, monetized health or environmental benefit of reducing an additional unit (ton) of emissions. A related metric used in environmental economics that offers a reverse perspective is marginal damage (MD), or the health or environmental damage incurred by emitting an additional ton of pollutant. MB is an important decision metric in economic evaluation of air pollution policies as it provides a direct indication of the rate-of-return on potential investments made in abating emissions. Mathematically, MB/MD is the derivative of the total damage curve (i.e., the overall, monetized societal impact of air pollution) with respect to emissions. MB for criteria air pollutants is traditionally depicted to decrease as emissions are progressively reduced (Goodstein 1995; Hussen 2004), indicating diminishing returns with each added ton of emission control. Such a negatively or downward-sloped MB curve with abatement is mathematically equivalent to a convex total damage curve with abatement. Convexity in this context implies that as emissions are reduced, the societal damage of air pollution initially declines rapidly, but with continued abatement, this rate-of-decrease in damage, or the accrued benefits from abatement, slows. This general behavior is attributed to the natural assimilative capacity of the environment to cleanse itself of pollution; a capacity that loses efficiency as the atmosphere becomes more polluted (Hussen 2004). At best, this is appropriate for some primary pollutants, but a downward-sloped MB curve may be an oversimplification for secondary pollutants whose production depends nonlinearly on the availability of emitted precursors. The prime example is ground-level ozone $\left(\mathrm{O}_{3}\right)$ 
formed from $\mathrm{NO}_{\mathrm{x}}\left(\mathrm{NO}+\mathrm{NO}_{2}\right)$ and volatile organic compounds (VOCs). At low levels of $\mathrm{NO}_{\mathrm{x}}$ emissions, removal of each ton of $\mathrm{NO}_{\mathrm{x}}$ is very effective in reducing $\mathrm{O}_{3}$, yielding a large, positive MB. At very high $\mathrm{NO}_{\mathrm{x}}$ emission levels, and with limited VOCs, reducing $\mathrm{NO}_{\mathrm{x}}$ may be counterproductive, leading to increased $\mathrm{O}_{3}$ concentrations through slower titration of $\mathrm{O}_{3}$ by NO (a negative MB or disbenefit) (Fann et al. 2009; Hakami et al. 2004; Muller and Mendelsohn 2009; Pappin and Hakami 2013A). This duality in $\mathrm{O}_{3}$ response to $\mathrm{NO}_{\mathrm{x}}$ control seen in extreme chemical environments presents a specific, well-known case for non-convexity and an upward-sloped MB curve.

While non-convexity is a long-established concept in other areas of environmental economics (Baumol and Bradford 1972), such as aquatic ecosystems (Crocker and Forster 1981) or environmental aesthetics (Anderson and Francois 1997; Randall et al. 1974), non-convexity in air pollution impacts is treated as an exception to the general rule (Fraas and Lutter 2011; Fraas and Lutter 2012; Hall and Hall 1997; Repetto 1987). Repetto (1987), using results from box model simulations with limited $\mathrm{O}_{3}$ chemistry, first suggested non-convexities in the response of $\mathrm{O}_{3}$ to precursor controls, but with a focus on $\mathrm{NO}_{\mathrm{x}}$-rich urban environments. Hakami et al. (2004) used regional high-order, forward sensitivity analysis in an air quality model to quantify local responses of $\mathrm{O}_{3}$ to domainwide precursor emission reductions. The authors found predominantly negative secondorder derivatives of $\mathrm{O}_{3}$ with respect to $\mathrm{NO}_{x}$ emissions, indicative of a non-convex response surface. Drawing upon previous studies (Fann et al. 2009; Muller and Mendelsohn 2009), Fraas and Lutter $(2011 ; 2012)$ later discussed the exceptional case of non-convexity in the presence of negative MBs or disbenefits that poses challenges in implementing economically efficient policy instruments. While indications of non- 
convexity exist in the literature, a general lack of efficient modeling tools, data, and resources has inhibited characterization of the $\mathrm{NO}_{\mathrm{x}} \mathrm{MB}$ curve to fully test the assumption of convexity. This work intends to characterize the $\mathrm{NO}_{\mathrm{x}} \mathrm{MB}$ curve on a source-by-source basis, and demonstrate that in the case of $\mathrm{NO}_{\mathrm{x}}$ and $\mathrm{O}_{3}$, non-convexity forms the general rule rather than the exception.

\subsection{Methods}

$\mathrm{NO}_{\mathrm{x}} \mathrm{MBs}$ are partly driven by the sensitivity of $\mathrm{O}_{3}$ to $\mathrm{NO}_{\mathrm{x}}$ that depends on the relative abundance of precursor species in the atmosphere. Characterization of the $\mathrm{NO}_{\mathrm{x}}$ MB curve necessitates the use of atmospheric models that adequately describe the nonlinear pathway from $\mathrm{NO}_{x}$ emissions to $\mathrm{O}_{3}$ concentrations, and can do so on a ton-byton basis. Furthermore, sources differ in their public health impacts based on their proximity to population centers and the atmospheric conditions conducive to local and downwind $\mathrm{O}_{3}$ formation. This combination of factors indicates that the same ton of $\mathrm{NO}_{\mathrm{x}}$ control for various sources may have different health impacts. Estimating MBs on a source-by-source level would thus yield invaluable information for air quality decisionmaking.

To estimate source-specific MBs, we use adjoint (or reverse) sensitivity analysis in a regional air quality model. The "reverse" or backward characteristic of the adjoint method indicates that influences on various receptors are traced back to individual sources through an auxiliary set of equations that govern source-receptor relationships. To accomplish this, the analysis entails backward (in time and space) integration of adjoint equations after forward simulations are carried out. More details about adjoint sensitivity analysis and its applications in health benefits assessment can be found 
elsewhere (Pappin and Hakami 2013A). Our approach simultaneously calculates sensitivities of model output with respect to a large number of input parameters (Hakami et al. 2006; Sandu et al. 2005). Achieving the same level of detail with traditional modeling approaches limits analyses to a few sources or groups of sources (Fann et al. 2009; Fann et al. 2012A; Mauzerall et al. 2005), or else requires simplifications of nonlinear chemical processes that may lead to underestimations of $\mathrm{NO}_{\mathrm{x}} \mathrm{MBs}$ (Muller and Mendelsohn 2007; Muller and Mendelsohn 2009; Muller and Mendelsohn 2012). Adjoint sensitivity analysis is an ideal tool for the purpose of this study as it allows for estimation of MBs for a multitude of polluters across different locations, sectors, and times while accounting for nonlinear atmospheric processes (Mesbah et al. 2013; Pappin and Hakami 2013A). We note that while the adjoint method offers sensitivity information on a sourceby-source basis, it cannot feasibly provide information about the distribution of impacts across receptors (a question more suitable to forward methods of sensitivity analysis). Adjoint sensitivity analysis is most appropriate for applications where a collective measure of policy effectiveness is sought, such as the total health or environmental damage of emissions, as in the case of seeking to estimate MBs.

We construct MB curves for mobile and point sources using the adjoint of the U.S. EPA's Community Multiscale Air Quality model, or CMAQ (Byun and Schere 2006). The gas-phase CMAQ-adjoint model used in this study is based on CMAQ v4.5.1 with the SAPRC-99 chemical mechanism (Carter 2000). The adjoint of CMAQ has been validated previously (Hakami et al. 2007) and used in various health impact studies (Mesbah et al. 2013; Pappin and Hakami 2013A; Pappin and Hakami 2013B; Zhao et al. 2013). We use the standard U.S. EPA domain spanning the continental U.S. at a $36-\mathrm{km}$ 
horizontal grid resolution with 34 vertical layers extending into the stratosphere. Our CMAQ-adjoint simulations are conducted over the $\mathrm{O}_{3}$ season of 2007 (May 1 September 30). Emissions are based on the National Emission Inventory (NEI) for the U.S. and the National Pollutant Release Inventory (NPRI) for Canada, and are generated using the Sparse Matrix Operator Kernel Emissions (SMOKE) model. Meteorological inputs are from the Weather Research and Forecasting (WRF) model, processed using the Meteorology Chemistry Interface Processor (MCIP). Performance evaluation of observed and simulated hourly $\mathrm{O}_{3}$ concentrations for the $2007 \mathrm{O}_{3}$ season indicates a mean fractional error (MFE) of $16 \%$ and mean fractional bias (MFB) of 2.5\%. Comparison of observed daily maximum $8 \mathrm{~h}$ average (DM8A) $\mathrm{O}_{3}$ concentrations with simulated DM8As (used for health impact estimation) yields a MFE of 15\% and MFB of 9.5\%.

We define $\mathrm{MB}$ as the monetary societal benefit (\$) of reducing $\mathrm{NO}_{\mathrm{x}}$ emissions by 1 ton from a given mobile or point source. We focus our analysis on the $\mathrm{MB}$ of $\mathrm{NO}_{\mathrm{x}}$ emission reductions, as $\mathrm{NO}_{\mathrm{x}}$ has by far the largest impact on population exposure to $\mathrm{O}_{3}$ of all precursor species (Pappin and Hakami 2013A). Our estimations of MB account for averted mortality in the U.S. population resulting from reduced short-term $\mathrm{O}_{3}$ exposure. We consider only acute $\mathrm{O}_{3}$ exposure mortality, and not acute morbidity, as mortality has a high monetary value and is the largest contributor to monetized health benefits of emission reductions (Hubbell et al. 2005). We do not account for environmental impacts, as we focus our analysis on population health damages. We consider only acute exposure mortality without consideration for mortality from long-term exposure to $\mathrm{O}_{3}$ based on the most recent assessment of the weight of evidence for causal associations between $\mathrm{O}_{3}$ and mortality (U.S. EPA 2013). As the overall behavior of $\mathrm{NO}_{\mathrm{x}} \mathrm{MBs}$ is driven largely by the 
chemistry of $\mathrm{O}_{3}$ production, we believe that the generality of our approach or results are not lost in exclusion of other $\mathrm{O}_{3}$ damage endpoints. We note that $\mathrm{NO}_{\mathrm{x}}$ emissions also contribute to $\mathrm{NO}_{2}$ exposure and inorganic PM formation, and that our MB estimates do not capture the full spectrum of impacts seen through species other than $\mathrm{O}_{3}$.

Adjoint estimation of $\mathrm{NO}_{\mathrm{x}} \mathrm{MBs}$ is based on the definition of a scalar adjoint cost function, $J$, as follows.

$$
J=V_{S L} \sum_{t} \sum_{\omega} M_{0 \omega} P_{\omega}\left(1-e^{-\beta \Delta C_{\omega t}}\right)
$$

Detailed description of the application of Equation 6.1 in the adjoint model is provided elsewhere (Pappin and Hakami 2013A). For MB estimation, $J$ is the monetized mortality count in the U.S. population attributable to short term $\mathrm{O}_{3}$ exposure over May 1 - September 30, i.e., the $2007 \mathrm{O}_{3}$ season $(\$), V_{S L}$ is the value of a statistical life, estimated to be $\$ 7.9$ million in $2008 \mathrm{USD}^{32}{ }^{3} M_{0 \omega}$ is the 2007 all-age, non-accidental mortality rate in location $\omega$ ( $\mathrm{yr}^{-1}$, scaled to a daily rate), and $P_{\omega}$ is the 2007 all-age population in location $\omega$, both of which are reported by the Centers for Disease Control and Prevention (CDC) at the county level; $\beta$ is the effect estimate derived from epidemiological studies; and $\Delta C_{\omega t}$ is the change in $\mathrm{DM} 8 \mathrm{~A} \mathrm{O}_{3}$ concentration at time $t$ and location $\omega$, with respect to a reference concentration of zero. We apply a $\beta$ of $4.27 \times 10^{-4} \mathrm{ppb}^{-1}$ for $\mathrm{DM} \mathrm{A} \mathrm{O}_{3}$ due to its wide coverage of populations across the U.S. (Bell et al. 2004).

We construct $\mathrm{MB}$ curves for 1 ton of emitted $\mathrm{NO}_{\mathrm{x}}$ using various U.S.-wide emission abatement scenarios. We use emission inventories for the $\mathrm{O}_{3}$ season of 2007 as our baseline of comparison. Abatement scenarios are defined by U.S.-wide, fixed 
percentage reductions in (a) mobile (onroad and nonroad) or point source emissions (e.g., a $20 \%$ reduction in all mobile source emissions only), or (b) both mobile and point source categories simultaneously (e.g., a $20 \%$ reduction in all mobile and point source emissions). Scenarios of 20, 40, 60, 80, and $100 \%$ reductions in emissions of all species from either source category are used. For each scenario, 2007 emissions are perturbed by a specified percentage in the forward CMAQ model. Concentration outputs from the forward model are used to calculate a new set of adjoint forcing terms (details are available elsewhere; Pappin and Hakami 2013A) and for calculating adjoint-based MBs in the backward model.

We categorize MBs for $\mathrm{NO}_{\mathrm{x}}$ emitted from (1) any mobile source and (2) any point source in a given grid cell in the domain. We report $\mathrm{MBs}$ for 1 ton of $\mathrm{NO}_{\mathrm{x}}$ emitted over the $\mathrm{O}_{3}$ season according to the spatiotemporal (i.e., day-to-day and layer-by-layer) distribution of emissions for any given source. Mobile source MBs are calculated for surface-level emissions only, while point source MBs are proportionally integrated over all vertical model layers whose emissions are non-zero, according to

$$
M B_{\omega}=\frac{\sum_{t} \sum_{z} \frac{\partial J}{\partial e_{\omega z t}} e_{\omega z t}}{\sum_{t} \sum_{z} e_{\omega z t}}
$$

where $M B_{\omega}$ is the $\mathrm{NO}_{\mathrm{x}} \mathrm{MB}$ for a mobile or point source in location $\omega$. MBs for a given grid cell are emission-weighted averages over all vertical model layers, $z$, and all simulation times, $t$, in that location. Adjoint sensitivities, $\partial J / \partial e_{\omega z t}$, are outputs of the CMAQ-adjoint model and indicate the sensitivity or response of U.S.-wide mortality to $\mathrm{NO}_{\mathrm{x}}$ emitted in location $\omega$, at layer $z$, for time $t$. Adjoint sensitivities are scaled to 
amount to the influence of 1 ton of $\mathrm{NO}_{\mathrm{x}}$ emitted over the $\mathrm{O}_{3}$ season. As the adjoint method provides sensitivity information specific to each location, layer, and time of emission release, the distinction between mobile and point source MBs comes from emission weighting in Equation 6.2.

When depicting U.S.-wide average MBs, we report the emission-weighted average MB in the U.S. for either mobile or point source categories. U.S.-wide average MBs are therefore most strongly influenced by MBs of large sources. When depicting

point source $\mathrm{MBs}$, we apply a filter to exclude small point sources $\left(\mathrm{NO}_{\mathrm{x}}\right.$ emissions $<100$ ton/season). We note that adjoint-based MBs indicate how emissions generated in one location contribute to a change in nationwide $\mathrm{O}_{3}$ exposure somewhere along their trajectory, but that the adjoint method cannot specify where such changes in exposure occur within the boundaries of the U.S (as defined by the adjoint cost function in Equation 6.1). Our estimations of $\mathrm{NO}_{\mathrm{x}} \mathrm{MBs}$ are therefore representative of the nationwide public health benefit in the U.S. attributed to a 1 ton reduction in $\mathrm{NO}_{\mathrm{x}}$ from a given source.

\subsection{Results and Discussion}

Mobile- and point-MBs across the U.S. are estimated to average $\$ 13,000$ and $\$ 14,000 /$ ton, respectively, at baseline 2007 emission levels (Figure 6.1A-B). For mobile sources, MBs at baseline 2007 emission levels range from $-\$ 86,000 /$ ton to $\$ 87,000$ per ton of $\mathrm{NO}_{\mathrm{x}}$ emitted near New York, NY and upwind of Los Angeles, CA, respectively (Figure 6.1A). For point sources whose baseline $\mathrm{NO}_{\mathrm{x}}$ emissions exceed 100 ton/season, $\mathrm{NO}_{\mathrm{x}} \mathrm{MBs}$ range from $-\$ 20,000$ to $\$ 39,000 /$ ton at baseline (Figure 6.1B). 

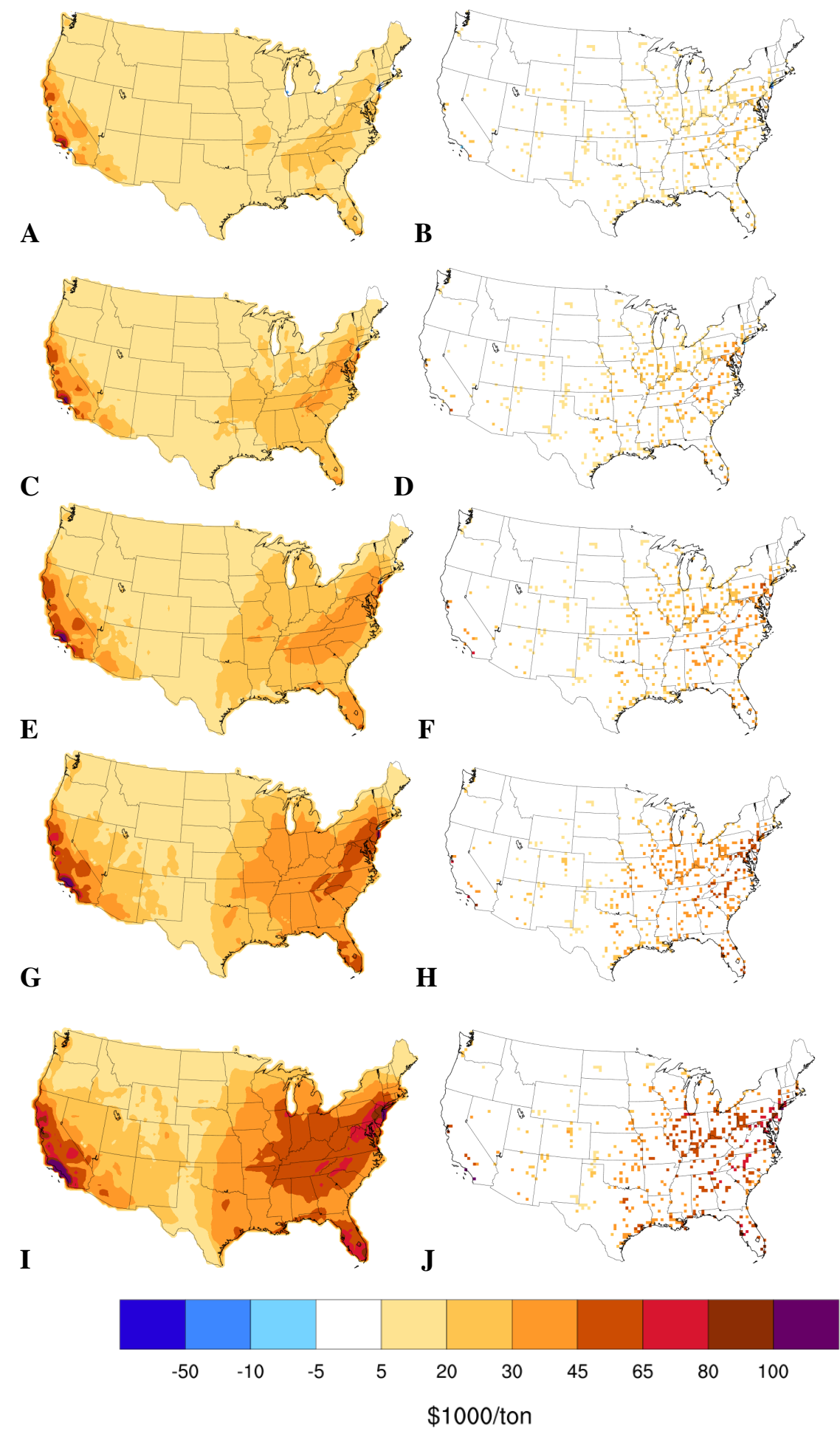

Figure 6.1 Simulated MBs for $\mathrm{NO}_{\mathrm{x}}$ emitted from mobile sources (left panel) and point sources (right panel) across the U.S. MBs are shown for baseline 2007 emission levels (A-B) and for U.S.-wide abatement of all species emitted from both mobile and point source categories in amounts of $40 \%$ (CD), $60 \%$ (E-F), $80 \%$ (G-H), and $100 \%$ (I-J). MBs are for 1 ton of $\mathrm{NO}_{\mathrm{x}}$ emissions allocated over the 
$2007 \mathrm{O}_{3}$ season (May-September) according to the spatiotemporal distribution of emissions. MB values are only shown for point sources $(B, D, F, H, J)$ whose emissions exceed 100 ton/season at baseline.

Our estimates at baseline are comparable to those found by others using various photochemical modeling tools and approaches (Fann et al. 2009; Fann et al. 2012A; Mauzerall et al. 2005; Mesbah et al. 2013; Pappin and Hakami 2013A). Mauzerall et al. (2005) used forward sensitivity analysis in an air quality model to estimate $\mathrm{NO}_{\mathrm{x}} \mathrm{MBs}$ through its $\mathrm{O}_{3}$ health impact. They found MBs for large point sources in the eastern U.S ranged from $\$ 10,700-\$ 52,800 /$ ton. We note that our MB estimates based on acute $\mathrm{O}_{3}$ health impacts are similar in magnitude to reported estimates of $\mathrm{PM}_{2.5}$-based MBs. Fann et al.(2009) used reduced-form air quality modeling to estimate $\mathrm{NO}_{\mathrm{x}} \mathrm{MBs}$ that account for chronic $\mathrm{PM}_{2.5}$ exposure mortality and morbidity in the U.S. population. In the study, $\mathrm{NO}_{\mathrm{x}} \mathrm{MBs}$ averaged $\$ 10,000 /$ ton for mobile sources and $\$ 9,700-15,000 /$ ton for point sources over 9 urban areas of the U.S., and estimates varied substantially by source location. A later study by Fann et al. (2012A) employed source apportionment in an air quality model to estimate average MBs (termed benefits-per-ton) that consider long-term health impacts over the U.S. They reported lower estimates averaging 4,500/ton for mobile source $\mathrm{NO}_{\mathrm{x}}$ and 3,700/ton for power plant $\mathrm{NO}_{\mathrm{x}}$. Although the overall public health burden of $\mathrm{PM}_{2.5}$ is larger than that of $\mathrm{O}_{3}$ (Lim et al. 2012), a comparison of our MB estimates with those reported previously suggests that $\mathrm{NO}_{\mathrm{x}}$ emissions may have the potential to incur as much damage through $\mathrm{O}_{3}$ in the short term as in the long term through $\mathrm{PM}_{2.5}$. Future research employing reverse sensitivity analysis of $\mathrm{PM}_{2.5}$ responses is necessary to further quantify the impact of $\mathrm{NO}_{\mathrm{x}}$ through different pathways. 
Mobile- and point-MBs are spatially heterogeneous at all levels of abatement and show similar behavior despite differences in the vertical layers of emission release (i.e., surface vs. elevated layers). Our findings therefore suggest that location is a stronger predictor of $\mathrm{O}_{3}$-based $\mathrm{NO}_{\mathrm{x}} \mathrm{MB}$ than source category.

The dominant feature in Figure 6.1 is the widespread increase in $\mathrm{NO}_{\mathrm{x}} \mathrm{MBs}$ towards higher levels of abatement. Without exception, positive MBs become more positive and MBs that are initially negative (i.e., disbenefits) become less so - and eventually positive - with U.S.-wide reductions in emissions. In other words, as the relative abundance of $\mathrm{NO}_{\mathrm{x}}$ declines with added controls, each additional ton of $\mathrm{NO}_{\mathrm{x}}$ reduction carries larger benefits than the previous ton. Such behavior is due to the role of $\mathrm{NO}_{\mathrm{x}}$ availability in $\mathrm{O}_{3}$ production in $\mathrm{NO}_{\mathrm{x}}$-rich environments. When $\mathrm{NO}_{\mathrm{x}}$ is abundant, competition between $\mathrm{NO}_{\mathrm{x}}$ molecules is high, yielding a small impact of increased $\mathrm{NO}_{\mathrm{x}}$ availability on $\mathrm{O}_{3}$. As less $\mathrm{NO}_{\mathbf{x}}$ becomes available for reactions to produce $\mathrm{O}_{3}$, additional $\mathrm{NO}_{\mathrm{x}}$ molecules face little competition and have higher $\mathrm{O}_{3}$ formation efficiency, yielding larger MBs. A transition from negative to positive MBs is an extreme example, where a unit of emission reduction that initially carries an overall disbenefit eventually reduces $\mathrm{O}_{3}$ exposure at some point further along the emission reduction trajectory. Positive MBs in Figure 6.1 are widespread across low- $\mathrm{NO}_{\mathrm{x}}$ environments in the U.S., while negative MBs are localized in $\mathrm{NO}_{\mathrm{x}}$-rich (or $\mathrm{NO}_{\mathrm{x}}$-inhibited) urban areas at baseline.

Depiction of MB as a function of emission reduction (abatement) level (a MB or MD curve) yields insight about the predicted benefits of added controls. A mix of MB curves for select, individual urban areas and point sources in the U.S. demonstrate the 
spectrum of behavior seen across different chemical environments in varying proximity to population centers (Figure 6.2).
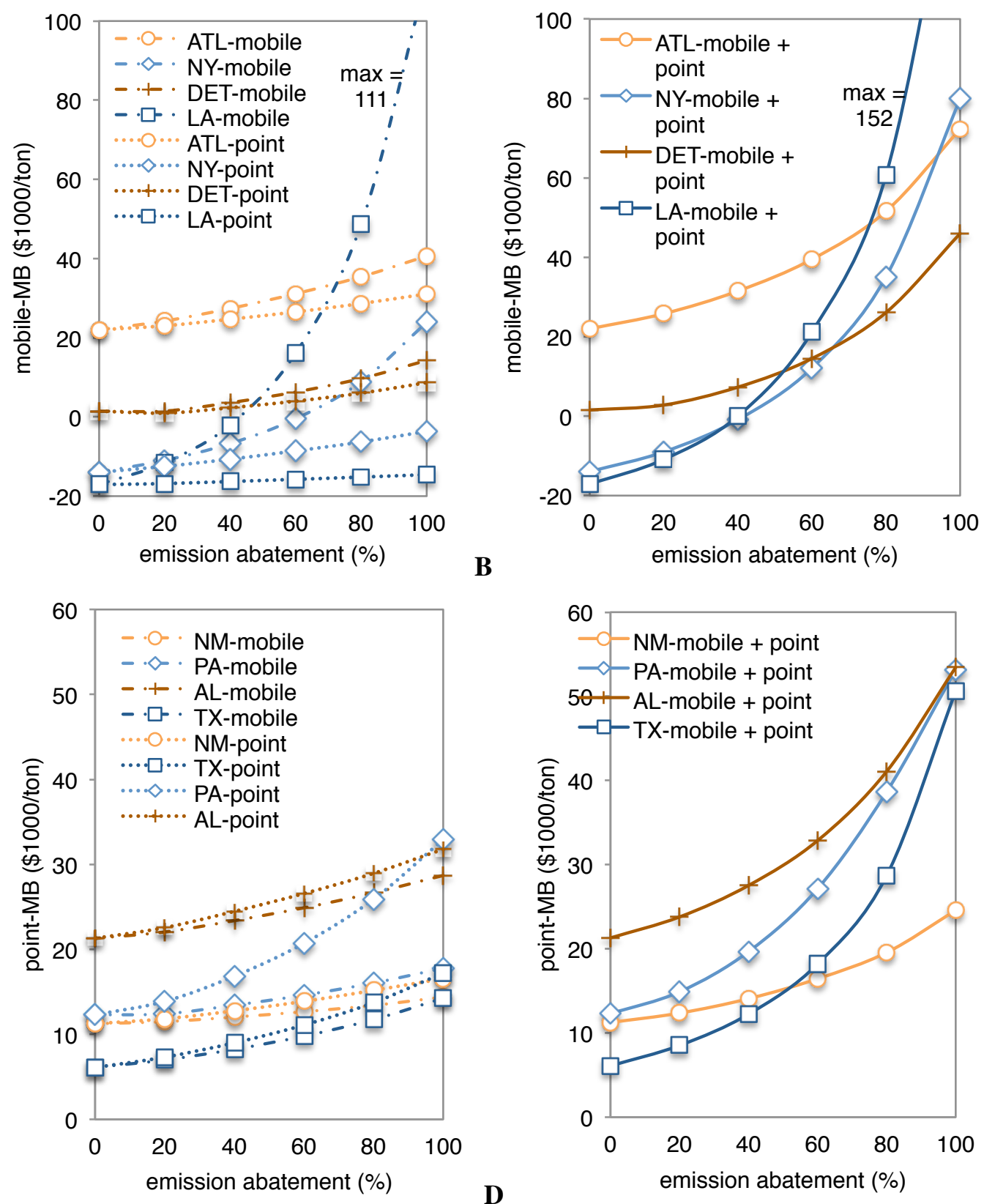

B

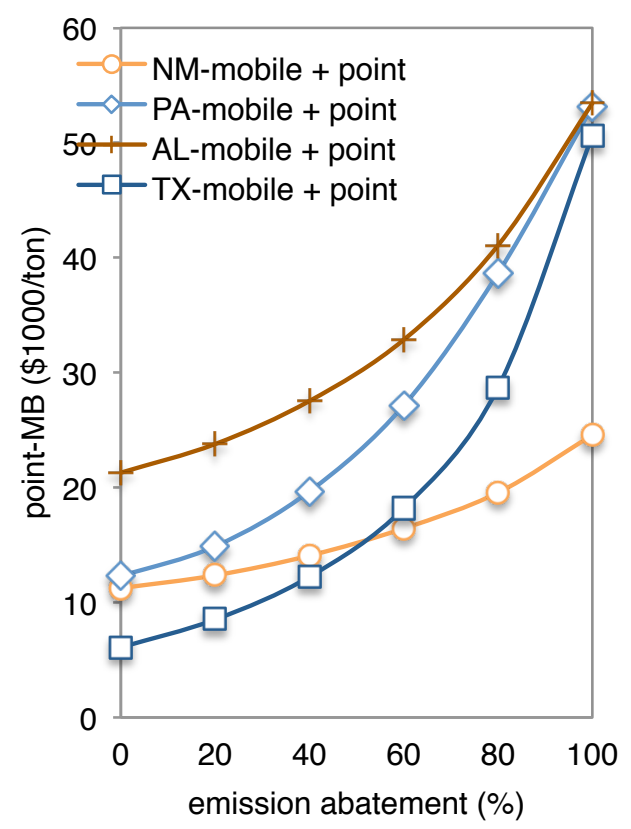

Figure 6.2 Simulated $\mathrm{NO}_{\mathrm{x}} \mathrm{MBs}$ as a function of U.S.-wide abatement level for a sample of source locations. Mobile-MBs (A-B) are the benefits associated with reductions in $\mathrm{NO}_{\mathrm{x}}$ emitted from mobile sources in a grid-cell within the specified city (Atlanta (ATL), New York (NY), Detroit (DET), Los Angeles (LA)). Point-MBs (C-D) are the benefit associated with reductions in $\mathrm{NO}_{\mathrm{x}}$ emitted from an 
anonymous, major point source in the specified state (NM, PA, AL, TX). Hatched and dashed lines (A, C) depict MBs for 0-100\% abatement of all species emitted from mobile or point sources, respectively, across the U.S., as compared to 2007 levels. Solid lines (B, D) show the same for simultaneous reductions in both mobile and point sources. For example, the "LA-point" dashed line in (A) shows mobile-MBs at different levels of U.S.-wide point source abatement.

Mobile source MBs (Figure 6.2A-B) and point source MBs (Figure 6.2C-D) rise invariably, monotonically, and nonlinearly as nation-wide emission levels decline from the 2007 baseline. MBs increase by 2-30 times their initial value, and by as much as $\$ 169,000 /$ ton with continued abatement of both source categories. The nonlinearity in the total damage function implied in these plots indicates a changing atmospheric regime as the abundance of $\mathrm{NO}_{\mathrm{x}}$ progressively declines. Such a shift can eventually amount to a change in MB sign (i.e., from negative to positive) for environments that are initially $\mathrm{NO}_{\mathrm{x}}$-inhibited. One example is a grid-cell in Los Angeles (LA in Figure 6.2A-B), whose mobile-MB at baseline is estimated to be $-\$ 17,000 /$ ton and grows rapidly to $\$ 152,000 /$ ton as mobile and point source emissions are abated completely (Figure 6.2B). Given that vehicles are by far the dominant source of anthropogenic emissions in and around LA, its MB is very sensitive to mobile source abatement (Figure 6.2A). MB behavior depicted for LA is among the most extreme of any source across the U.S. due to (1) the initially $\mathrm{NO}_{\mathrm{x}}$-inhibited environment necessitating a transition through the $\mathrm{O}_{3}$ ridge into a $\mathrm{NO}_{\mathrm{x}}{ }^{-}$ limited regime with abatement, (2) the large populations in and downwind of LA, and (3) the lack of large point sources in the region that lends little change in MB with point source abatement (Figure 6.2A). The spectrum of behavior depicted in Figure 6.2 shows that as $\mathrm{NO}_{\mathrm{x}}$ approaches background levels, changes in MBs become more drastic with each additional unit of abatement. 
System-wide average $\mathrm{MB}$ curves represent the overall response of $\mathrm{O}_{3}$ health damages in the U.S. population to a 1 ton reduction in $\mathrm{NO}_{\mathrm{x}}$ from an average emitter. We calculate system-wide average mobile- and point-MB curves for separate and combined reductions in source categories (Figure 6.3). System-wide average MBs are calculated using emission-weighted averaging of MBs in Figure 6.1 for all sources for any given abatement scenario. On an aggregate level, MBs are positive, upward-sloping, and rise from baseline monotonically and nonlinearly with $\mathrm{NO}_{\mathrm{x}}$ emission controls of increasing intensity. Contrary to traditional depictions of $\mathrm{MB}$ curves, $\mathrm{NO}_{\mathrm{x}} \mathrm{MBs}$ increase substantially as background concentrations are approached, indicating a heightened sensitivity of pristine environments to any added $\mathrm{NO}_{\mathrm{x}}$. With combined reductions in both mobile and point source categories, $\mathrm{NO}_{\mathrm{x}} \mathrm{MBs}$ increase roughly 3-4 times (from $\$ 13,000$ to $\$ 51,000 /$ ton for mobile-MB, and from $\$ 14,000$ to $\$ 45,000 /$ ton for point-MB) after $100 \%$ emission abatement. When examined separately by source category, mobile-MBs are more sensitive to abatement of mobile source emissions, while point-MBs are similarly affected by controls of either source category. 


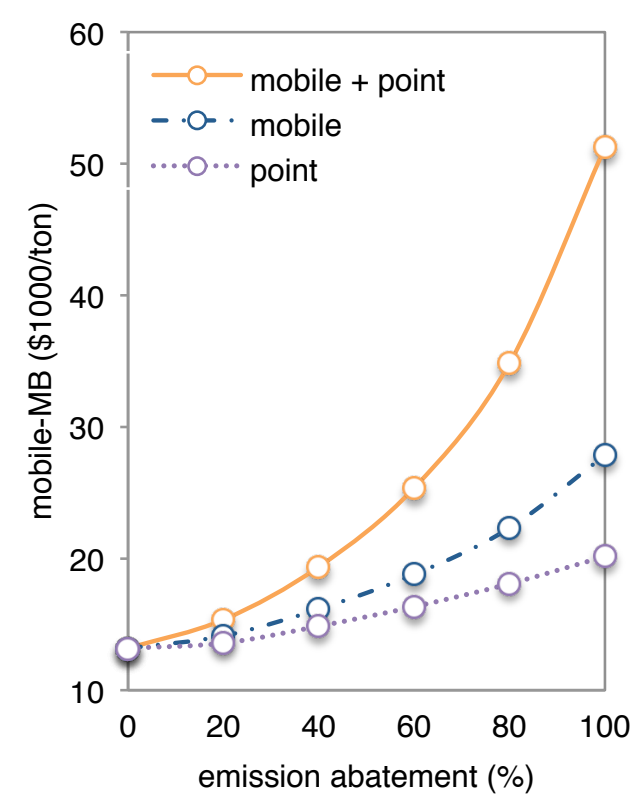

A

Figure 6.3 Average U.S.-wide mobile- (A) and point-MB curves (B) for various levels of U.S.-wide abatement of all species emitted from mobile and point sources. Average MB curves are depicted as a function of mobile source abatement (hatched line) and point source abatement (dashed line) separately. Solid lines depict the combined rise in MBs from controlling both source categories simultaneously. MBs shown here are emission-weighted averages over the U.S.

The prevalent presumption of a downward-sloping MB curve in the environmental economics literature is akin to convexity of the cumulative or total damage curve with respect to abatement. Total damage in this context is the monetized U.S. health burden from $\mathrm{O}_{3}$ exposure at a given abatement level. Our estimations of $\mathrm{MB}$ curves indicate a consistently concave $\mathrm{NO}_{\mathrm{x}}$ total damage curve with compounding benefits towards lower levels of emissions (Figure 6.4). In other words, the total damage depicted in Figure 6.4 declines more rapidly towards higher levels of abatement. Past studies have assumed that MBs for a specific source do not change with $\mathrm{NO}_{\mathrm{x}}$ emissions, and estimated total damage by multiplying fixed MBs and emissions (Muller and 
Mendelsohn 2007; Muller et al. 2011; Banzhaf et al. 2004). This linear approximation of the total damage curve is prone to underestimation as it neglects its curvature as emissions change. Our finding of a strictly concave total damage curve applies to all sources, rather than to specific cases of sources with negative MBs at baseline as suggested previously (Fraas and Lutter 2011). Further, our findings suggest a smooth and gradual transition in $\mathrm{O}_{3}$-based $\mathrm{NO}_{\mathrm{x}}$ benefits across chemical regimes, contrary to cases with discontinuities or instantaneous changes in damages suggested by others (Goodstein 1995; Montgomery 1972).

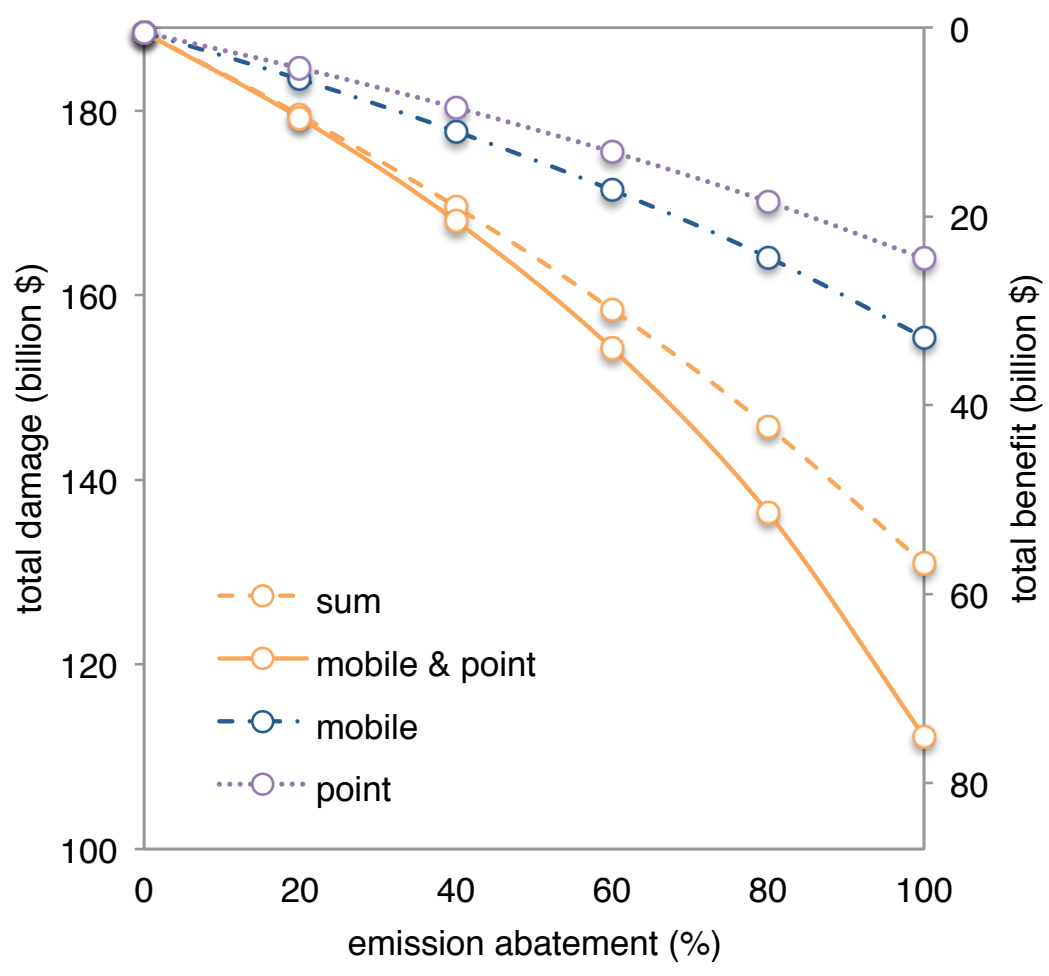

Figure 6.4 Total U.S.-wide damage as a function of U.S.-wide abatement of mobile sources (hatched line), point sources (short-dashed line), and both simultaneously (solid line). Total damage is the monetized health burden of mortality attributable to short-term $\mathrm{O}_{3}$ exposure of the U.S. population, calculated at each abatement level. Total benefit shown on the secondary axis is the avoided health damage in moving from the 2007 baseline to lower emission levels. The long-dashed line depicts total 
benefits as the summation of benefits incurred from controlling mobile and point source emissions separately (i.e., the summation of benefits for the short-dashed and hatched lines). Total damage accounts for $\mathrm{O}_{3}$ exposure during the $2007 \mathrm{O}_{3}$ season (May-September).

Closer examination of Figures 6.3-6.4 illustrates an important point about nonlinearity and curvature of the total damage function. The benefits of controlling both mobile and point source categories together (solid line in Figure 6.4) are larger than the summation of benefits incurred from controlling these sources separately (long-dashed line in Figure 6.4). This nonadditivity is a result of the concave nature of the $\mathrm{NO}_{\mathrm{x}}$ total damage curve that becomes more pronounced as the overall abundance of $\mathrm{NO}_{\mathrm{x}}$ declines. Combined reductions in both mobile and point sources, together rather than separately, results in a more extreme $\mathrm{NO}_{\mathrm{x}}$-limited environment where each additional ton of $\mathrm{NO}_{\mathrm{x}}$ gains higher efficiency for $\mathrm{O}_{3}$ production. In the presence of regional-to-national scale emission controls from many polluters across different sectors, a simple addition to estimate the overall benefits of abatement is likely to underestimate the combined effect. We note that the quantitative results shown in this work are based on emission reduction scenarios that apply nation-wide, fixed percentage reductions in point and/or mobile source emissions. For a specific policy targeting only a subset of sources (e.g., on-road gasoline vehicles), $\mathrm{NO}_{\mathrm{x}} \mathrm{MBs}$ would increase with abatement, but at a lower rate. Our results also show that evaluating such policy options in isolation from the larger emission reduction landscape is likely to (significantly) underestimate the benefits of abatement.

Benefit-cost assessment relies on estimates of MB and the cost-per-ton of emission reduction (referred to as marginal [abatement] cost, or MC) as decision-making metrics. Based on economic equilibrium theory, the net societal benefit of a given policy 
item is highest when MB equals MC ( $\mathrm{A}^{*}$ in Figure 6.5) (Fann et al. 2011; Goodstein 1995; Tietenberg 1995). At lower abatement levels than this equilibrium point (to the left of $\mathrm{A}^{*}$ in Figure 6.5), there is incentive to further control emissions as the benefit exceeds the cost incurred from reducing an additional ton of emissions. At higher abatement levels than $\mathrm{A}^{*}$, rising costs are prohibitive and no longer compensated in full by expected returns. Traditional depictions of this equilibrium point assume a downward-sloped MB curve and an upward-sloped MC curve with abatement. Our findings of an upwardsloping and monotonic $\mathrm{NO}_{\mathrm{x}} \mathrm{MB}$ curve challenge the conventional scheme presented in Figure 6.5 in two important ways. First, if the MB curve is upward-sloping and nonlinear, as in Figures 6.2-6.3, the uniqueness of the equilibrium point, as often presumed in the environmental economics literature, is not guaranteed and will depend on the shape of the total damage and cost curves (Fraas and Lutter 2012). Second, in the presence of an upward-sloped MB curve, an economically viable abatement policy at baseline (i.e., $\mathrm{MB}>\mathrm{MC}$ ), would yield a new intersection point that lies at a higher abatement level (or lower emission level; $\mathrm{A}_{\text {new }}$ in Figure 6.5 ) than that suggested by a conventionally convex total damage curve. Our results, in most cases, are therefore in support of more stringent emission reduction targets than previously thought to be economically efficient. One example is the emission cap of the U.S. cap-and-trade program. Upward-sloping MB curves, such as those found in this study, would provide economic incentive for a lower system-wide emission cap than previously envisioned. The general shape of the MB curve in Figure 6.5 is taken from our results (i.e., Figure 6.3); however, we emphasize that it is a qualitative depiction of system-wide behavior of benefits and costs of control. Though strictly qualitative, Figure 6.5 demonstrates that a shift in the economic 
paradigm, from convexity to non-convexity, would entail an important change in the MB curve, and a correspondingly significant shift in the point of economic equilibrium.

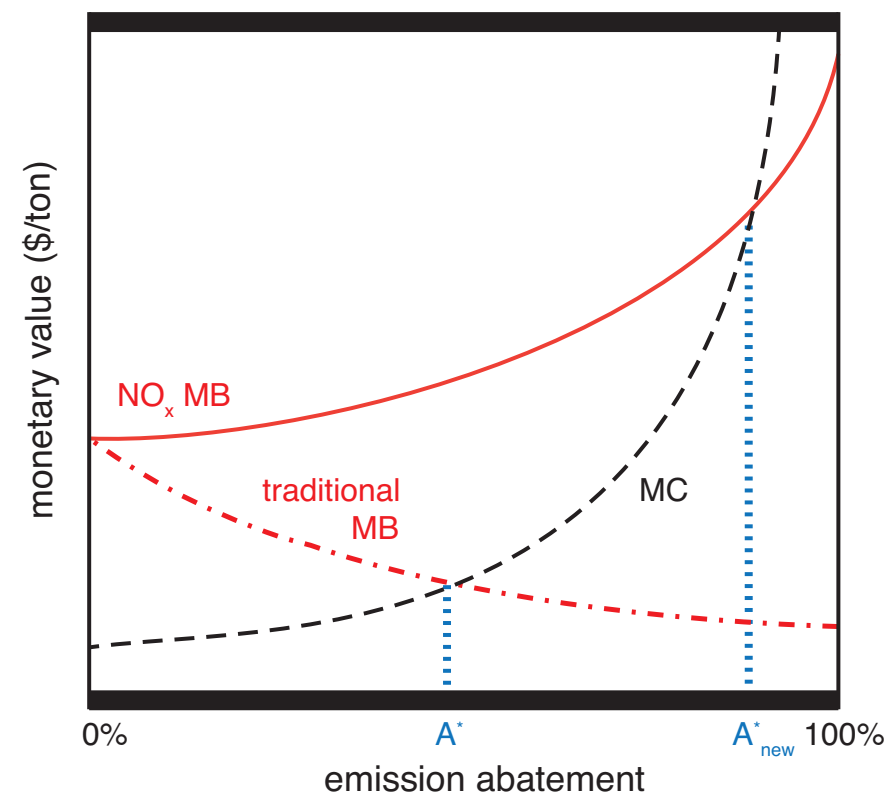

Figure 6.5 Depiction of the economic equilibrium point $\left(\mathrm{A}^{*}\right)$ between $\mathrm{MB}$ and $\mathrm{MC}$ (dashed line) based on traditional forms of MB curves (hatched line) and our findings (solid line). We consider 2007 as our baseline and the starting point for MB curves. Curves shown here are qualitative and for demonstrative purposes only and are based on the general shape of the system-wide average curves in Figure 6.3. Note that the baseline-level $M C$ is often less than the $M B$, and changes in the shape of either curve will affect where the points of equilibrium lie.

We note that our conclusions apply generally to the overall system and not necessarily to each source individually, as the shapes of MB and MC curves differ from source to source. We also recognize that the MB curves presented here are based on a series of U.S.-wide emission reductions and are therefore projections of responses of MBs to national rather than local changes in emission patterns. Reductions in emissions from single sources, in most cases, would have little tangible impact on the ambient availability of $\mathrm{NO}_{\mathrm{x}}$ in the system when other emissions are kept constant. $\mathrm{MB}$ curves for 
single sources are thus expected to be relatively flat compared to the curvature seen in Figures 6.2-6.3. Changes in sectoral emissions, such as mobile or electricity generating sources, seldom happen in isolation and commonly materialize within a broader, nationwide context. As such, we believe that our depiction provides a more realistic and relevant view of MB behavior for decision-making. In the particular case of regulating sources with negative MBs, a broader consideration of system- or sector-wide abatement and resulting benefits is preferable to isolating the impacts of abatement of individual sources. Information garnered from the total damage/benefit curve, such as those in Figure 6.4, can therefore yield important insight into the cumulative benefits of widespread emission control policies.

The concave (or nonconvex) behavior demonstrated here is for $\mathrm{NO}_{\mathrm{x}}$ damage function based only on mortality from short-term $\mathrm{O}_{3}$ exposure. More comprehensive estimation of $\mathrm{NO}_{x} \mathrm{MBs}$ would consider non-fatal health and other environmental impacts of $\mathrm{O}_{3}$, particularly endpoints related to long-term exposure. In addition to influencing $\mathrm{O}_{3}$ production, $\mathrm{NO}_{\mathrm{x}}$ also contributes to secondary PM formation, and inclusion of PMrelated damage endpoints may affect the overall shape of the MB curve. Though no studies have fully tested the assumption of convexity as applied to PM health damages, indications of non-convexity through two pathways exist in the literature. The first is non-convexity due to the role of chemical equilibrium in formation of secondary inorganic PM constituents from $\mathrm{NO}_{\mathrm{x}}$. Fann et al. (2012A) reported consistently higher MBs for all inorganic PM precursor emissions under a 2016 abatement scenario compared to estimates for 2005 emission levels. It is noteworthy that the authors found (slightly) increased MBs even for primary emissions of PM, possibly due to nonlinearity 
induced by other species through aerosol growth and dynamics. Holt et al. (2015) compared PM sensitivities to $\mathrm{NO}_{\mathrm{x}}, \mathrm{SO}_{2}$, and $\mathrm{NH}_{3}$ emissions in 2005 and 2012 and found that for $\mathrm{SO}_{2}$ and $\mathrm{NO}_{\mathrm{x}}$, sensitivities increase with emission controls (2012 levels). Zhang et al. (2012) used the high-order direct decoupled method (HDDM; Hakami et al. 2003) to estimate $2^{\text {nd }}$ order derivatives of PM with respect to precursor emissions, including $\mathrm{NO}_{\mathrm{x}}$. They found mostly negative $2^{\text {nd }}$-order HDDM sensitivities to precursor emissions, indicating a concave response surface. In addition to indications of a non-convex atmospheric response of $\mathrm{PM}$ to $\mathrm{NO}_{x}$, recent studies have suggested that unlike $\mathrm{O}_{3}$ (Bell et al. 2006), a non-linear and concave concentration-response function may be more suitable for $\mathrm{PM}_{2.5}$ (e.g., Burnett et al. 2014; Pope et al. 2011), implying an epidemiologically induced non-convex damage curve (Apte et al. 2015; Goodkind et al. 2014; Pope et al. 2015). A log-linear or supralinear (concave) curve implies a large slope, or high incremental risk per unit increase in concentration, at low levels of exposure that diminishes towards higher concentrations. Such a shape of the concentration-response function indicates a heightened sensitivity of populations to PM in cleaner environments. Combined with the likely non-convex atmospheric response of $\mathrm{PM}$ to $\mathrm{NO}_{\mathrm{x}}$, persisting, or even enhanced concavity, may be expected with inclusion of PM in the damage function.

Our MB estimates are affected by uncertainties stemming from atmospheric modeling and emissions characterization, population demographics, epidemiological concentration-response relationships, and economic valuation of damage endpoints. Firstly, we estimate MB curves at a 36-km horizontal resolution that may not capture fine spatial gradients in $\mathrm{O}_{3}$ exposure, particularly over urban and suburban areas. Second, we use population and mortality data for 2007 without considering dynamic changes in 
population that may become relevant into the future. Third, we apply a uniform effect estimate to the entire U.S. population, while recognizing that effect estimates may vary by region (Bell et al. 2004). We also assume a linear, no-threshold response of mortality to $\mathrm{O}_{3}$ exposure based on the current epidemiological literature (Bell et al. 2006). Alternate forms of the concentration-response function would affect $\mathrm{NO}_{\mathrm{x}} \mathrm{MB}$ estimation along the abatement trajectory. Fourth, while we assign a uniform value of a statistical life for valuating public health impacts, this willingness-to-pay may differ among subgroups of the population and shift as pollution levels and consumer preferences change. We note that we use 2007 emissions as our reference point, and MBs at current emission levels, or those under planned policies, may differ from estimates reported here, particularly given the progressive post-2007 emission reductions that have taken place (NEI 2016). We also note that our estimates of $\mathrm{NO}_{\mathrm{x}} \mathrm{MBs}$ consider the impact of $\mathrm{NO}_{\mathrm{x}}$ control on the U.S. population only. In reality, emissions generated within the U.S. may also impact public health in other nations (Anenberg et al. 2009; Pappin and Hakami 2013A), and thus marginal reductions in emissions may have additional monetary benefits not captured here. Interpretation of our results should consider these uncertainties and limitations of our analysis.

Our findings suggest compounding benefits of progressive $\mathrm{NO}_{\mathrm{x}}$ emission reductions. In an important case, the benefit of urban $\mathrm{NO}_{\mathrm{x}}$ control has been debated for cities with negative MBs at current emission levels, where localized emission reductions appear unfavorable in the short-term (Fraas and Lutter 2012; Pappin and Hakami 2013A). Compounding benefits with added $\mathrm{NO}_{\mathrm{x}}$ control on a broader scale support continued $\mathrm{NO}_{\mathrm{x}}$ abatement in the longer term for urban air quality management. Strictly concave total 
damage functions and upward-sloping MB curves with abatement, such as those found here, suggest larger yet unexplored economic incentives for more aggressive emission reductions.

Given the challenges that $\mathrm{O}_{3}$ and $\mathrm{PM}$ pose to air quality management in North America and the world over, we believe that the notion of generally non-convex behavior for secondary pollutants such as $\mathrm{O}_{3}$ has important policy implications. Reported emission trends from the U.S. EPA suggest that anthropogenic $\mathrm{NO}_{\mathrm{x}}$ emissions have decreased by more than 30\% from 2007 to 2014 (NEI 2016). Based on our results, this level of reduction could place us at the onset of an important point in time and on the MB curve, where $\mathrm{NO}_{x}$ MBs can increase significantly in the near future (Figure 6.4). In such a policy context, adhering to the traditional view of convexity and disregarding the compounding nature of $\mathrm{NO}_{\mathrm{x}}$ control benefits does not appear to be a prudent option. 


\section{Chapter: Compounding Benefits in the Presence of Epidemiological and Atmospheric Nonlinearities}

This chapter has been published in Environmental Research Letters under the title "Health Benefits of Reducing $\mathrm{NO}_{x}$ Emissions in the Presence of Epidemiological and Atmospheric Nonlinearities". ${ }^{2}$ It is reproduced in full in this chapter with minor editorial changes with permission under the creative commons copyright license. ${ }^{3}$ It consists of original research for which Amanda Pappin is the main contributor (80\%).

\subsection{Introduction}

Managing the public health burden of ambient air pollution is a complex undertaking informed by atmospheric science and engineering, health, economic, and policy disciplines. Synthesis of information from these fields can yield insight into the public health impacts of air pollution, playing a critical role in science-based decisionmaking. Perhaps the most tangible form of quantitative assessments is one that links public health with sources of emissions themselves, yielding direct decision metrics. One useful metric in this context is benefit-per-ton (BPT; synonymous with marginal benefit/damage), which refers to the monetized health benefit of reducing 1 ton of emissions at their source. The BPT metric applies monetary value to aggregate health damages (such as mortality or morbidity counts) attributed to a 1 ton change in pollutant emissions. The utility of such a metric lies in its ability to be directly compared with the

\footnotetext{
${ }^{2}$ Pappin AJ, Hakami A, Blagden P, Nasari M, Szyszkowicz, Burnett RT. 2016. Health benefits of reducing $\mathrm{NO}_{\mathrm{x}}$ emissions in the presence of epidemiological and atmospheric nonlinearities. Environ Res Lett 11:064015.

${ }^{3}$ License information can be found at https://creativecommons.org/licenses/by/3.0/.
} 
cost-per-ton of emission reduction (emission abatement) in a benefit-cost assessment framework.

Numerous, complex atmospheric processes act on emitted species to transport and transform them into pollutants such as $\mathrm{O}_{3}, \mathrm{NO}_{2}$, and $\mathrm{PM}_{2.5}$ at the point of exposure. The potential for secondary pollutant formation often depends nonlinearly on the abundance of precursor species in ambient air. Nonlinearity in atmospheric processes implies that the same ton of emission control would yield different health impacts, and different BPT estimates, at different levels of emissions or emissions abatement. Such nonlinearity in BPT estimates with emissions abatement has been discussed in the literature for $\mathrm{O}_{3}$ and its precursors (Hakami et al. 2004; Pappin et al. 2015A; Repetto 1987). A recent study by Pappin et al. (2015) found increasing BPT estimates for $\mathrm{NO}_{\mathrm{x}}$ control in the U.S. with widespread and progressive emissions abatement, due solely to nonlinear atmospheric chemistry and for acute $\mathrm{O}_{3}$ exposure mortality using a single-pollutant model. In other words, contrary to the commonly held view in environmental economics (Goodstein 1995; Hussen 2004), each additional ton of $\mathrm{NO}_{\mathrm{x}}$ emission control makes future abatement efforts more rewarding. While sufficient evidence of compounding benefits with abatement exists for $\mathrm{O}_{3}$ and $\mathrm{NO}_{\mathrm{x}}$, nonlinearity in the $\mathrm{PM}_{2.5}$ response to precursor emissions has not been studied to the same extent. Collectively in the literature, there are indications that a similar case of compounding benefits may hold for $\mathrm{PM}_{2.5}$ (Fann et al. 2012A; Holt et al. 2015; Zhang et al. 2012), though further research is needed to support this assertion.

In addition to a nonlinear atmospheric response, a second, and potentially significant, source of nonlinearity is that induced by concentration-response functions 
(CRFs). CRFs describe the relationship between pollutant concentrations and excess risk of a health endpoint. Traditionally, CRFs are modeled as linear functions, with logtransformed relative risk/hazard ratio (RR/HR) being linear with respect to concentration (Figure 7.1A). A linear in concentration model choice implies a constant increased risk per unit change in exposure, independent of the level of exposure itself. An alternate form of CRF found in the epidemiological literature is a supralinear curve, where the excess risk per unit concentration (i.e., the slope of the CRF) is highest at low levels of exposure, and gradually declines as the environment becomes more polluted (Figure 7.1B). Henceforth, we refer to this form as a log-concentration CRF (log-transformed RR/HR is a linear function of log-transformed concentration). Such a supralinear CRF has been recently proposed as a more fitting model choice for $\mathrm{PM}_{2.5}$ (Burnett et al. 2014; Crouse et al. 2015; Pope et al. 2011) and $\mathrm{NO}_{2}$ in Canada (Crouse et al. 2015) for mortality and other endpoints. A log-concentration CRF implies an increased sensitivity of populations in clean environments to any changes in $\mathrm{PM}_{2.5}$ or $\mathrm{NO}_{2}$ exposure. 


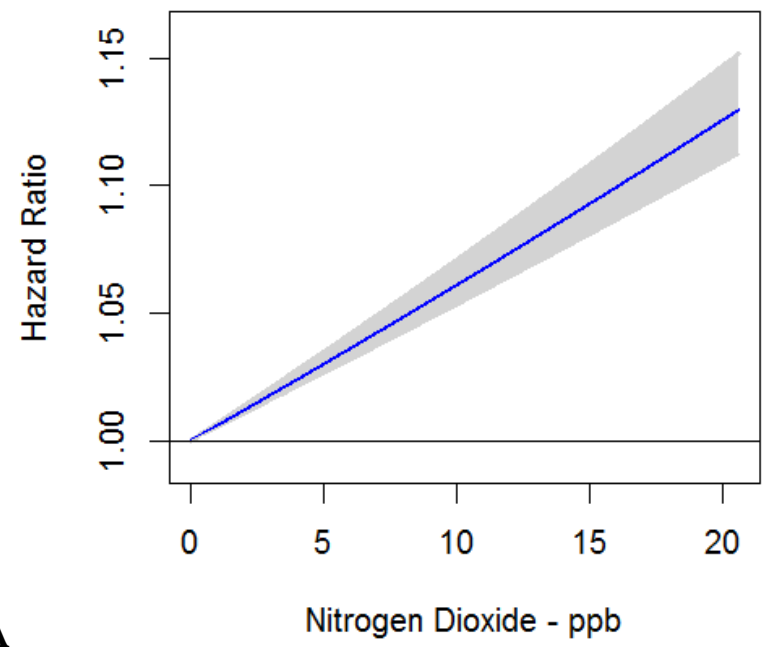

A

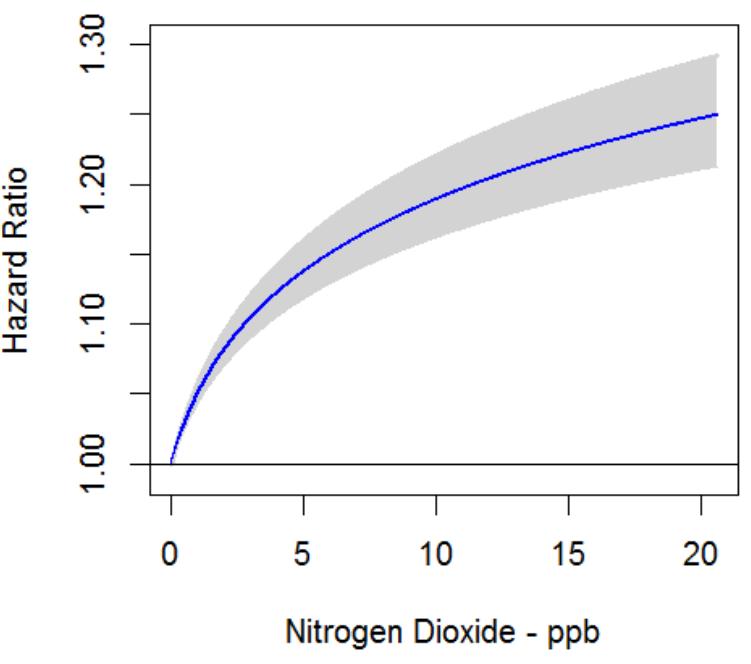

Figure 7.1 Examples of linear and supralinear forms of CRFs. CRFs depicted here are for $\mathrm{NO}_{2}$ based on a recent analysis of CanCHEC and are (A) linear in concentration and (B) of the logconcentration form.

Complexities in atmospheric processes necessitate the use of sophisticated models that adequately describe the potentially nonlinear pathway from sources of emissions to exposed populations. A general lack of efficient modeling methods has prevented full characterization of BPTs in the literature for various sources across a range of abatement levels/scenarios. Further, no previous study has examined both the atmospheric response of ambient concentrations to emissions and the shape of the CRF as potential sources of 
nonlinearity in the health benefits of abatement (e.g., Pappin et al. 2015A; Pope et al. 2015). We aim to examine the role of both factors in predicting the BPTs of $\mathrm{NO}_{\mathrm{x}}$ emission control in Canada.

\subsection{Methods}

We investigate how different forms of the CRF influence the health benefits of emission control. We do so using an atmospheric chemical transport model (CTM) run for 2007 emissions and meteorological conditions in North America and various, hypothetical emissions control or abatement scenarios. We use exposure estimates from a forward CTM simulation, combined with linear in concentration and log-concentration CRFs, to inform a set of adjoint (reverse or backward) simulations. The utility of an adjoint model is in its ability to estimate BPTs of emission control on a source-by-source basis (Pappin and Hakami 2013A). We account for the public health impacts of $\mathrm{O}_{3}$ and $\mathrm{NO}_{2}$ exposure in Canada, but not $\mathrm{PM}_{2.5}$, as the adjoint model for PM processes is still under development. While the public health burden of $\mathrm{PM}_{2.5}$ exceeds that of $\mathrm{O}_{3}(\mathrm{Lim}$ et al. 2012), $\mathrm{NO}_{\mathrm{x}}$ emissions have a significant impact on Canadian mortality due to $\mathrm{O}_{3}$ and $\mathrm{NO}_{2}$ exposure (Pappin and Hakami 2013A). Furthermore, Crouse et al. (2015) demonstrate that both $\mathrm{O}_{3}$ and $\mathrm{NO}_{2}$ contribute additional mortality risk to that predicted by $\mathrm{PM}_{2.5}$ in a large, nationally representative Canadian cohort.

Our focus is on chronic exposure mortality, as recent epidemiological studies suggest that long-term exposure to criteria pollutants poses a substantially higher risk of mortality than short-term exposure (Crouse et al. 2012; Jerrett et al. 2009; Krewski et al. 2009). We recognize that evidence for a causal association between $\mathrm{NO}_{2}$ exposure and mortality is an evolving area of research. A recent risk assessment for ambient $\mathrm{NO}_{2}$ found 
the collective evidence to be suggestive of a causal association between $\mathrm{NO}_{2}$ exposure in the long-term and mortality (Health Canada 2016). Two other analyses of CanCHEC (Crouse et al. 2015) and the American Cancer Society Cancer Prevention Study II cohort (Turner et al. 2016) found $\mathrm{NO}_{2}$ to be an independent predictor of mortality, adding to the body of evidence supporting an $\mathrm{NO}_{2}$ effect on mortality.

\subsubsection{Adjoint Formulation}

Our estimates of abatement health benefits account for non-accidental mortality from long-term $\mathrm{O}_{3}$ and $\mathrm{NO}_{2}$ exposure in Canada. We apply both linear and logconcentration CRFs for non-accidental mortality based on, though not identical to, a recent analysis of the Canadian Census Health and Environment Cohort (CanCHEC) (Crouse et al. 2015). As mortality is the largest contributor to the total, monetized societal benefits of improving air quality (Hubbell et al. 2005; Hubbell et al. 2006; U.S. EPA 1999), we consider our estimates to be largely representative of total health benefits.

CanCHEC is a population-based Canadian cohort consisting of subjects $>25$ years of age who filed the long-form census in 1991. It has been linked to the Canadian Mortality Database from the time of enrollment through 2006. Participants' exposure estimates for summertime $\mathrm{O}_{3}$ are from a combination of ground monitoring observations and atmospheric modeling predictions for 2002-2009 (Robichaud and Ménard 2014). Annual mean $\mathrm{NO}_{2}$ concentrations are derived from a national land use regression model for 2006 (Hystad et al. 2011). Exposures are assigned to each subject's postal code based on annual income tax data from 1984-2006 (Peters et al. 2013). Several individual-level covariates are included in the Cox Proportional Hazards Model that relates mortality to known risk factors such as income, education, and occupation, in addition to contextual 
risk factors representing both city and neighbourhood characteristics. More detailed information about analysis of CanCHEC can be found elsewhere (Crouse et al. 2012; Crouse et al. 2015; Peters et al. 2013).

Mathematically, BPTs estimated using adjoint sensitivity analysis are the derivatives of a cumulative health damage function with respect to emissions in each grid-cell location. In our case, this damage function, termed the adjoint cost function, is the monetized mortality count attributable to air pollution in Canada for a given exposure surface. For a linear in concentration CRF, the adjoint cost function is of the form in Equation 7.1,

$$
\mathrm{J}=\mathrm{V}_{\mathrm{SL}} \sum_{\omega} \mathrm{M}_{0, \omega} \mathrm{P}_{\omega}\left(1-\mathrm{e}^{-\beta \overline{\mathrm{C}}_{\omega}}\right)
$$

For a log-concentration CRF, the adjoint cost function takes the form,

$$
\mathrm{J}=\mathrm{V}_{\mathrm{SL}} \sum_{\omega} \mathrm{M}_{0, \omega} \mathrm{P}_{\omega}\left(1-\mathrm{e}^{-\beta \ln \left(\overline{\mathrm{C}}_{\omega}+1\right)}\right)
$$

We note that Equations 7.1-7.2 are written separately for $\mathrm{O}_{3}$ and $\mathrm{NO}_{2}$ using risk parameter estimates from the Cox proportional hazards model consisting of both pollutants together. In both cases, $J$ is the monetized number of non-accidental mortalities attributable to long-term $\mathrm{O}_{3}$ or $\mathrm{NO}_{2}$ exposure, per year, in Canada. We apply a value of statistical life $\left(V_{S L}\right)$ of 7.17 million dollars (2013 CAD) to each premature death in Equations 7.1-7.2 based on that used in Health Canada's Air Quality Benefits Assessment Tool (AQBAT; Judek et al. 2006). Above, $M_{0, \omega}$ is the non-accidental mortality rate for populations $>25$ years of age and $P_{\omega}$ is the population over 25 years of age, both for grid- 
cell location $\omega$ (derived from AQBAT). We apply risk coefficients, $\beta$, based on Cox proportional hazards models that imply linear regression for $\mathrm{O}_{3}$ and linear or log-linear regression for $\mathrm{NO}_{2}$ (Table 7.1). In Equations 7.1-7.2, $\bar{C}_{\omega}$ is the model-based, ground-level concentration of $\mathrm{O}_{3}$ or $\mathrm{NO}_{2}$ (in ppb) in grid-cell location $\omega$, averaged over all simulation days. We use an 8-hr averaging period for $\mathrm{O}_{3}$ (daily maximum 8-hr average; DM8A) and a 24-hr (daily) averaging period for $\mathrm{NO}_{2}$ for consistency with the exposure metrics used in analyzing CanCHEC.

Due to the computational cost of conducting full-year simulations, our CTMbased exposure estimates for $\mathrm{O}_{3}$ and $\mathrm{NO}_{2}$ are for the May-September period and relate to CRFs derived using summertime $\mathrm{O}_{3}$ and annual average $\mathrm{NO}_{2}$. Since we seek to attribute chronic exposure mortality to sources of emissions, we assume that our simulation period yields $\mathrm{O}_{3}$ and $\mathrm{NO}_{2}$ exposure levels typical of the May-September period in Canada, and would hence represent long-term exposure levels and source-receptor relationships.

Table 7.1 Risk estimates and CRFs from the CanCHEC study used in estimating BPTs ${ }^{\mathrm{a}}$.

\begin{tabular}{ll}
\hline $\begin{array}{l}\text { Two-Pollutant } \\
\text { Model Form }\end{array}$ & Cox Proportional Hazards Model ${ }^{\mathrm{b}}$ \\
\hline $\begin{array}{l}\text { Linear in } \mathrm{O}_{3} \\
\text { Linear in } \mathrm{NO}_{2}\end{array}$ & $\ln \mathrm{HR}=0.0027 \overline{\mathrm{C}}_{\mathrm{O}_{3}}+0.0059 \overline{\mathrm{C}}_{\mathrm{NO}_{2}}+$ covariates \\
\hline $\begin{array}{l}\text { Linear in } \mathrm{O}_{3}{ }^{\mathrm{c}} \\
\text { Log- } \mathrm{NO}_{2}\end{array}$ & $\ln \mathrm{HR}=0.0026 \overline{\mathrm{C}}_{\mathrm{O}_{3}}+0.0732 \ln \left(\overline{\mathrm{C}}_{\mathrm{NO}_{2}}+1\right)+$ covariates \\
${ }^{a}$ Models for non-accidental mortality, 25-89 years of age \\
${ }^{\mathrm{b}} \overline{\mathrm{C}}_{\mathrm{O}_{3}}$ used in deriving the CRFs is the summertime average DM8A O $\mathrm{O}_{3}$ concentration; $\overline{\mathrm{C}}_{\mathrm{NO}_{2}}$ is the annual \\
average $\mathrm{NO}_{2}$ concentration \\
${ }^{c}$ The risk estimate of 0.0026 for $\mathrm{O}_{3}$ from the two-pollutant log-NO $\mathrm{NO}_{2}$ model is used for estimating BPTs
\end{tabular}

Adjoint sensitivity analysis requires differentiation of the cost function (Equations 7.1-7.2) with respect to the local, hourly concentration. This differentiation yields the 
adjoint forcing term $(\phi)$, a key input parameter to an adjoint model used to calculate sensitivities (Hakami et al. 2007). For a linear in concentration CRF, the adjoint forcing term is formulated as in Equation 7.3,

$$
\phi_{\omega}=\frac{\mathrm{V}_{\mathrm{SL}} \mathrm{M}_{0, \omega} \mathrm{P}_{\omega} \beta \mathrm{e}^{-\beta \overline{\mathrm{C}}_{\omega}}}{\overline{\mathrm{tn}}}
$$

The adjoint forcing term for a log-concentration CRF is formulated as,

$$
\phi_{\omega}=\frac{\mathrm{V}_{\mathrm{SL}} \mathrm{M}_{0, \omega} \mathrm{P}_{\omega} \beta\left(\overline{\mathrm{C}}_{\omega}+1\right)^{-(\beta+1)}}{\overline{\mathrm{tn}}}
$$

where all variables are as defined before, $\bar{t}$ is the number of hours in the daily exposure metric ( 8 for DM8A $\mathrm{O}_{3}$ and 24 for daily average $\mathrm{NO}_{2}$ ), and $n$ is the number of simulation days (153 for the May-September $\mathrm{O}_{3}$ season).

\subsubsection{Case Study}

We use the U.S. EPA's Community Multi-scale Air Quality model (CMAQ; Byun and Schere 2006) and its gas-phase adjoint tool (CMAQ-adjoint, version 4.5.1; Hakami et al. 2007) to conduct our analysis. Our $\mathrm{O}_{3}$ and $\mathrm{NO}_{2}$ exposure surfaces for Canada are spatially resolved to $36 \mathrm{~km}$ using forward CMAQ simulations that inform a 
set of reverse calculations in the adjoint model. Using the CMAQ-adjoint model, we estimate monetized $\mathrm{O}_{3}$ and $\mathrm{NO}_{2}$-related health benefits, attributed to an incremental (1 ton) reduction in emissions (BPTs; $\$$ ton). We report BPTs of $\mathrm{NO}_{\mathrm{x}}$ control from sources in every grid-cell location, for various abatement scenarios. Our simulations are conducted over a domain spanning southern Canada, the continental U.S., and northern Mexico. We conduct our simulations over the $\mathrm{O}_{3}$ season of 2007 (May 1-September 30).

To estimate nonlinearity in BPTs with abatement, we define scenarios of widespread reductions in emissions from anthropogenic sources, using 2007 as the baseline from which we reduce emissions. For each scenario, we use fixed-percentage abatement $(0 \%, 25 \%, 50 \%, 70 \%, 85 \%)$ of all species emitted from anthropogenic sources within our domain. As biogenic emissions are mainly a function of meteorological conditions, we consider them to be constant in our analysis. We note that we do not perturb emissions outside of North America, as the inflow of pollution into our domain boundaries is constant from scenario to scenario.

\subsection{Results and Discussion}

As we aim to examine the role of atmospheric chemistry and the shape of the CRF on BPT estimates, we examine $\mathrm{O}_{3}$ and $\mathrm{NO}_{2}$ health impacts separately. When isolating the impacts of $\mathrm{NO}_{\mathrm{x}}$ emissions on either species, we apply the risk coefficient for only that species (Table 7.1) in the adjoint cost function and forcing terms [Equations (7.1-7.4)]. We note that as the coefficients used in BPT calculations are from twopollutant models with no interaction, our BPT estimates can be considered additive. 


\subsection{1 $\mathrm{NO}_{2}$-based Benefits-per-ton Based on Linear in Concentration and Log- Concentration CRFs}

At baseline, BPTs of $\mathrm{NO}_{\mathrm{x}}$ control are the estimated Canadian public health benefits of reducing an additional ton of $\mathrm{NO}_{\mathrm{x}}$ emitted from a specific source location (Figure 7.2) in 2007. For example, an estimated value of $\$ 50,000 /$ ton in Figure 7.2 indicates that 1 ton of $\mathrm{NO}_{\mathrm{x}}$ control in the specified location would yield estimated societal health benefits of $\$ 50,000 /$ ton in Canada. We note that Figure 7.2 depicts sources of influence, but does not provide information on the distribution of these health benefits within Canada.

We first present health benefits due to averted $\mathrm{NO}_{2}$ chronic exposure mortality. BPTs based on a linear in concentration CRF for $\mathrm{NO}_{2}$ (Figure 7.2A) are localized around populous Canadian cities and surrounding suburban areas. BPT estimates are highly variable within Canada due to the short lifetime of $\mathrm{NO}_{2}$ formed from emitted $\mathrm{NO}_{\mathrm{x}}$. BPTs for major cities in Canada vary with the size of populations susceptible to $\mathrm{NO}_{2}$ exposure from emitted $\mathrm{NO}_{\mathrm{x}}$. For example, $\mathrm{NO}_{\mathrm{x}}$ control in Vancouver incurs an estimated benefit of $\$ 460,000 /$ ton, while $\mathrm{NO}_{\mathrm{x}}$ control in Ottawa incurs a $\$ 270,000 /$ ton benefit (based on a linear in concentration CRF; Figure 7.2A).

In comparison to the traditional, linear in concentration form, BPTs based on a log-concentration $\mathrm{CRF}$ for $\mathrm{NO}_{2}$ show greater spatial coverage (Figure 7.2B). BPT estimates are almost consistently larger for the log-concentration CRF, particularly in cleaner or rural environments with low $\mathrm{NO}_{2}$ exposure levels. For example, $\mathrm{NO}_{\mathrm{x}}$ control in Vancouver and Ottawa incurs estimated benefits of $\$ 510,000$ and $\$ 500,000 /$ ton, respectively, with a log-concentration CRF. These BPT estimates are higher than those 
based on a linear in concentration CRF. Of the two cities, Ottawa shows a stronger contrast between CRFs due to its low $\mathrm{NO}_{2}$ concentrations. Low $\mathrm{NO}_{2}$ exposure levels fall within the region of the log-concentration CRF that incurs a high incremental risk per ppb. This increased risk is larger than what would exist at the same exposure level in the linear in concentration CRF. Highly populous urban areas of Canada present an opposite case. One example is $\mathrm{NO}_{\mathrm{x}}$ emitted in downtown Toronto, whose BPT is $\$ 840,000 /$ ton based on the traditional linear form of CRF. Use of a log-concentration CRF for Toronto leads to a significantly lower estimate of $\$ 650,000 /$ ton. Such lower BPTs based on a logconcentration $\mathrm{CRF}$ are due to high $\mathrm{NO}_{2}$ concentrations in and around Toronto, where the increased risk per ppb is lower than in the linear form. $\mathrm{NO}_{\mathrm{x}}$ emission control in Toronto translates into Canadian public health benefits both locally and downwind as $\mathrm{NO}_{2}$ levels decline; impacts that are collectively captured in our estimates of BPTs.

The general finding of more widespread benefits of $\mathrm{NO}_{\mathrm{x}}$ control for a logconcentration CRF indicates that the benefits of emission controls in both urban/suburban and rural areas should be considered as viable policy incentives. We note that while urban $\mathrm{NO}_{\mathrm{x}}$ control results in lower BPT estimates under a log-concentration model in 2007, BPTs will eventually rise with continued reduction in $\mathrm{NO}_{2}$ concentrations in the urban environment. 

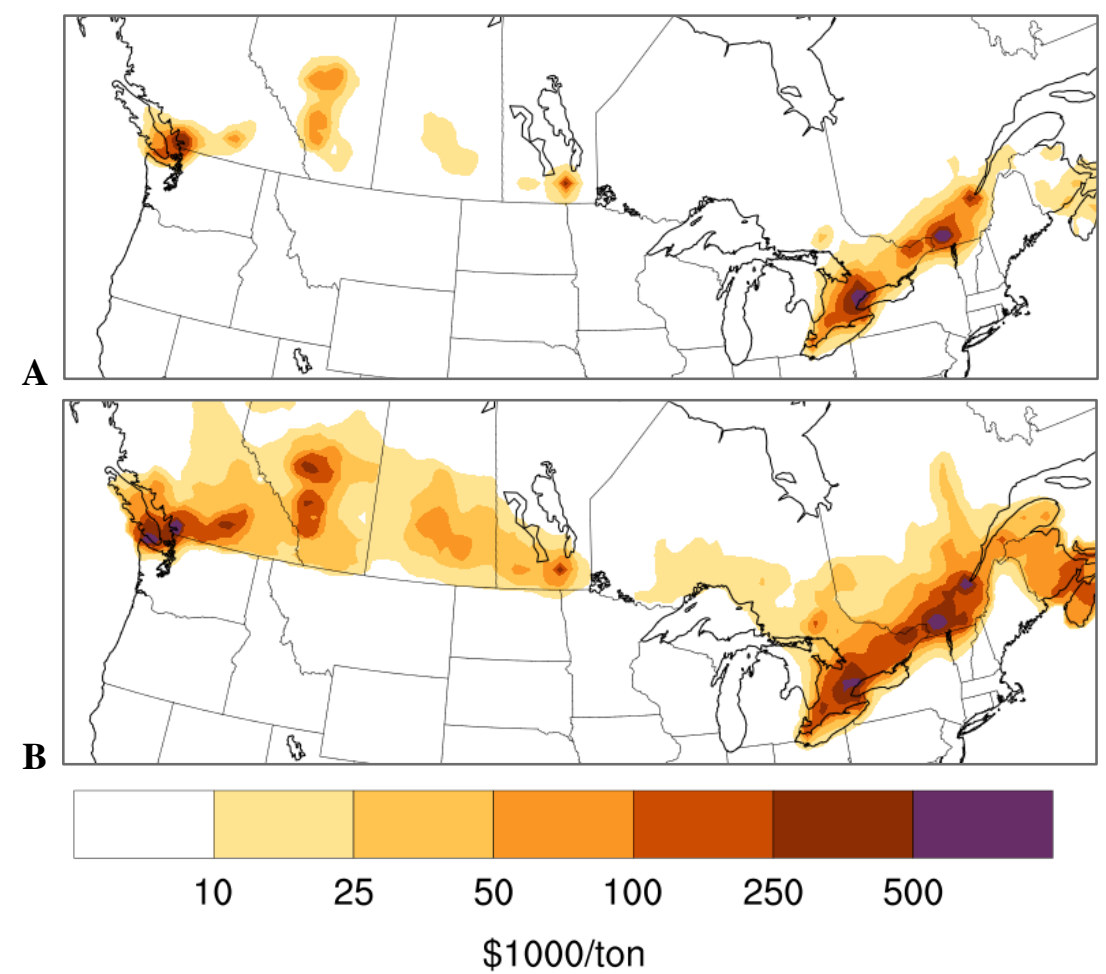

Figure 7.2 $\mathrm{NO}_{2}$-based BPTs for (A) a linear in concentration and (B) a log-concentration CRF at baseline 2007 emission levels. Estimates shown are the Canadian health benefits of reducing 1 ton of $\mathrm{NO}_{\mathrm{x}}$ emitted from ground level in each location $(\$ 1000 /$ ton $)$. BPT estimates are based on (A) $\beta=$ 0.0059 and $(\mathrm{B}) \boldsymbol{\beta}=\mathbf{0 . 0 7 3 2}$ for $\mathrm{NO}_{2}$ using two-pollutant models.

The choice of CRF is expected to play a more significant role in BPT estimates towards lower exposure levels or stricter emissions control policies than 2007. Dynamic changes in BPT estimates with abatement are therefore of interest, particularly as emissions in North America have been declining (ECCC 2014; U.S. EPA 2015). In order to isolate the impact of nonlinearity in the $\mathrm{CRF}$ and/or atmospheric response on BPT estimation, we define various abatement scenarios for which we assume unchanging population and mortality rates.

We find that BPTs are fairly constant, regardless of emission level, when a linear CRF for $\mathrm{NO}_{2}$ is used. Constant BPTs imply that $\mathrm{NO}_{2}$ concentrations change near linearly 
with $\mathrm{NO}_{\mathrm{x}}$ emissions. We therefore consider BPTs based on a linear in concentration CRF for $\mathrm{NO}_{2}$ at the 2007 baseline (Figure 7.2A) to be representative of BPTs at all abatement scenarios. As the atmospheric response of $\mathrm{NO}_{2}$ to $\mathrm{NO}_{\mathrm{x}}$ emissions is near linear, changes in BPT estimates across different abatement scenarios (Figure 7.3) can be attributed to nonlinearity induced by the CRF. We find that a log-concentration $\mathrm{CRF}$ for $\mathrm{NO}_{2}$ leads to increasing BPT estimates with more stringent abatement scenarios (Figure 7.3). This behavior exists due to the growing change in risk per unit concentration as $\mathrm{NO}_{2}$ exposure levels decline with continued emissions abatement under the log-concentration form of CRF (Figure 7.1B). For example, with large-scale, North American-wide emission reductions of $50 \%$, the benefit of $\mathrm{NO}_{\mathrm{x}}$ control in Toronto is estimated to be $\$ 1,250,000 /$ ton (Figure 7.3C); a 2-fold increase from $\$ 650,000 /$ ton for the 2007 baseline scenario (Figure 7.3A). At 25\% abatement of 2007 emissions, Toronto's BPT lies at an estimated $\$ 870,000 /$ ton (Figure 7.3B). 

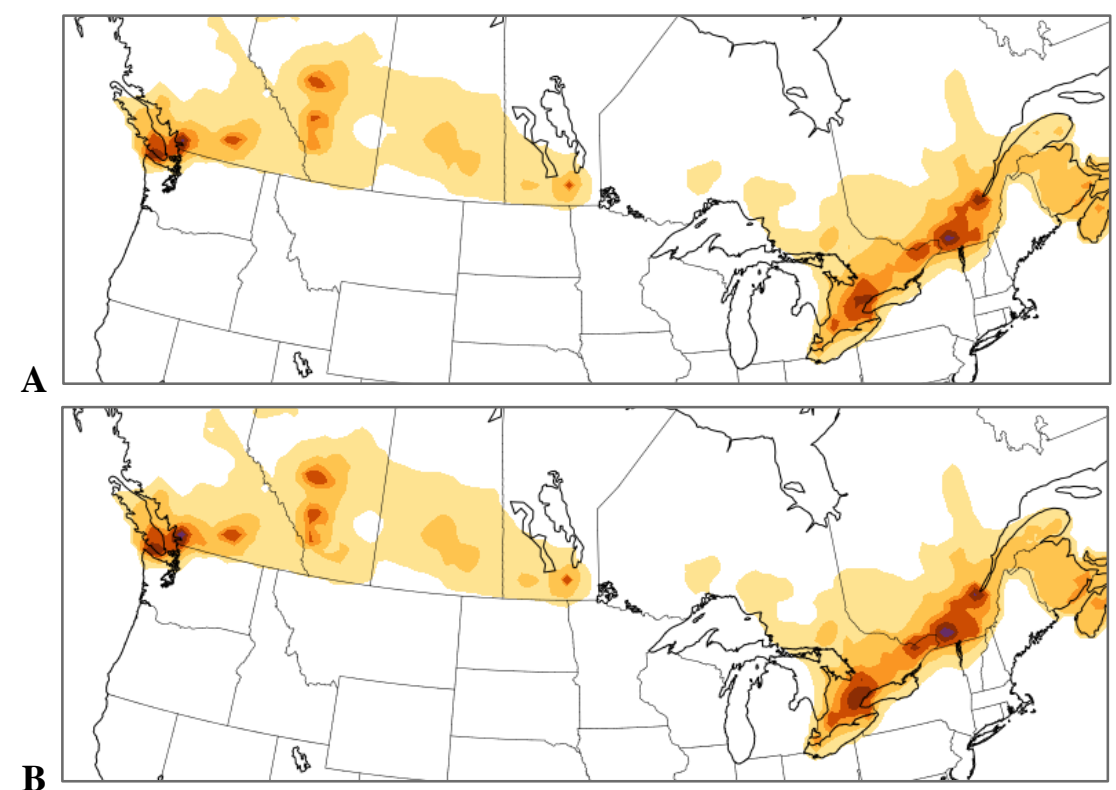

B

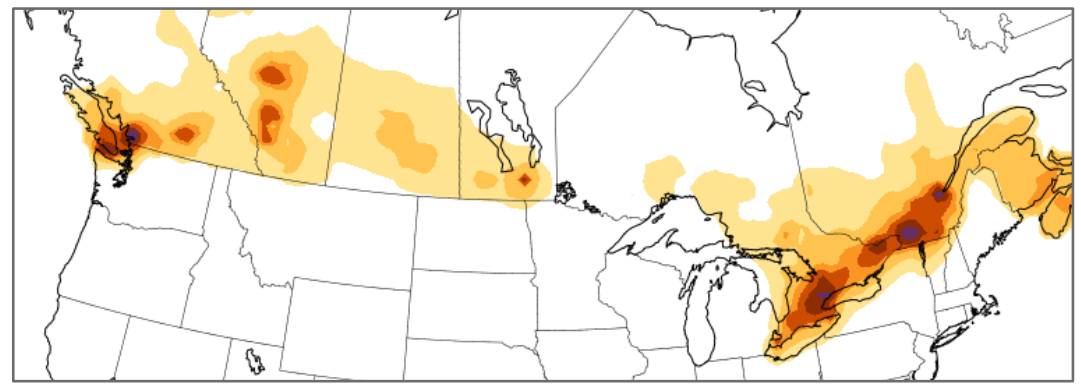

C

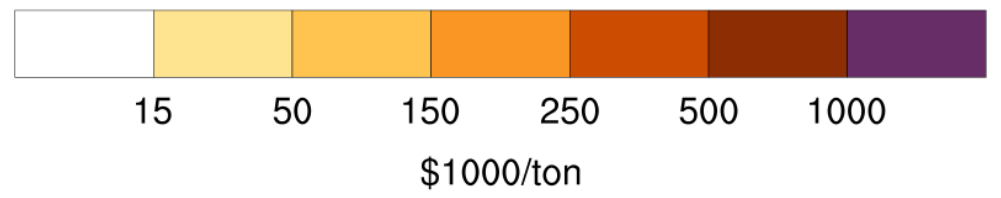

Figure 7.3 BPTs of $\mathrm{NO}_{\mathrm{x}}$ control based on a log-concentration CRF for $\mathrm{NO}_{2}(\beta=0.0732)$ from the two-pollutant model. BPTs are estimated at (A) 2007 emission levels and (B) for $25 \%$ and (C) $50 \%$ abatement scenarios. Abatement refers to the percentage reduction in emissions from all anthropogenic sources in North America, with 2007 emission levels as the baseline reference. 


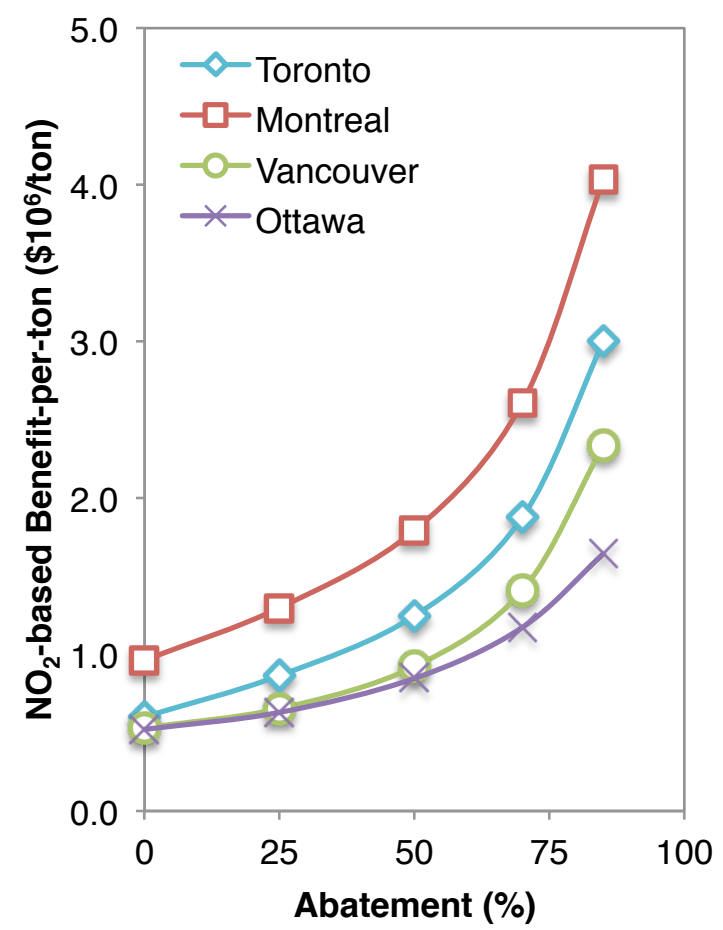

A

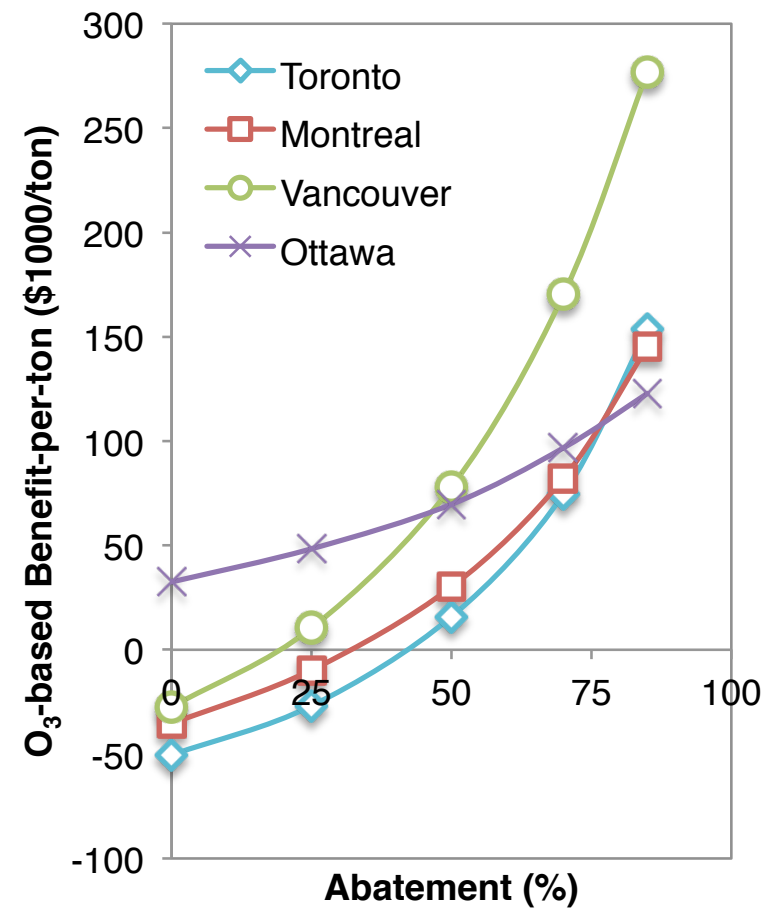

B

Figure 7.4 BPT estimates for ground-level sources located in four major cities in Canada. Estimates are based on (A) a log-concentration $\mathrm{CRF}$ for $\mathrm{NO}_{2}$ and (B) a linear in concentration $\mathrm{CRF}$ for $\mathrm{O}_{3}$. 
A collocated, pair-wise comparison of BPT estimates based on linear in concentration and log-concentration CRFs for $\mathrm{NO}_{2}$ yields insight into the predicted behavior among different statistical model choices (Figure 7.5). At baseline 2007 emission levels, BPTs based on a log-concentration CRF for $\mathrm{NO}_{2}$ are generally larger than those based on a linear in concentration CRF (Figure 7.5A). Further examination by population density (denoted by the color of markers in Figure 7.5) indicates that BPTs for $\mathrm{NO}_{\mathrm{x}}$ emitted in high-population areas (yellow/orange) are comparable between the two models. On the other hand, BPTs in cleaner, low-population areas (dark blue) estimated with a log-concentration CRF are considerably larger than those based on the traditional, linear form. This trend arises because $\mathrm{NO}_{2}$ exposure levels in these environments are on the low end of the spectrum of exposure levels in Canada, where the increased risk per ppb is heightened. This observed trend is similar among all levels of abatement, as populous areas always have higher $\mathrm{NO}_{2}$ concentrations than rural areas under blanket, fixed-percentage abatement scenarios. Going towards higher abatement levels (i.e., 85\%; Figure 7.5B), differences between the linear in concentration and log-concentration BPT estimates become more pronounced. 


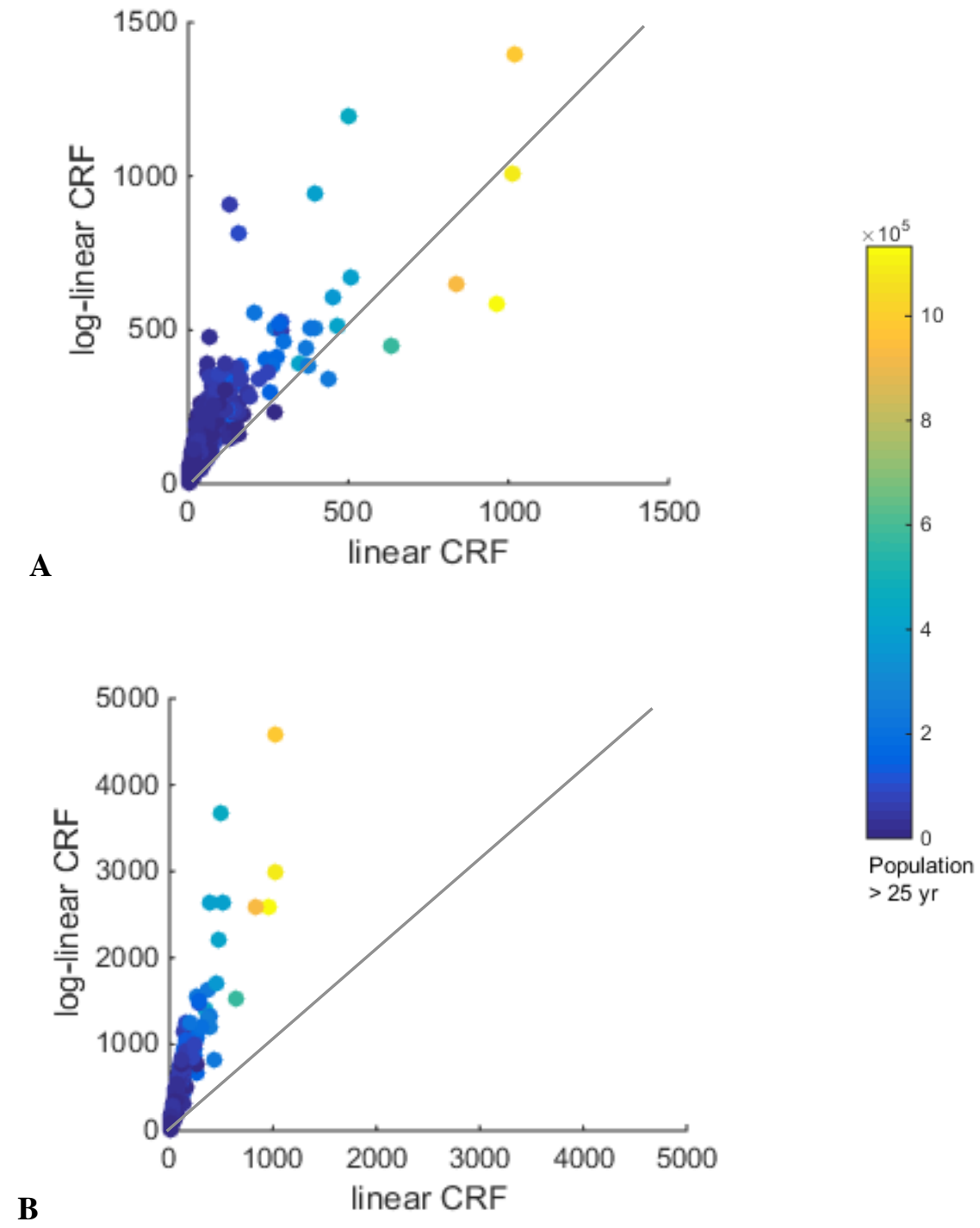

Figure 7.5 BPT estimates based on log-concentration vs linear in concentration CRFs for $\mathrm{NO}_{2}$. BPT estimates are shown for (A) baseline 2007 emission levels and (B) an $85 \%$ abatement scenario. Each point represents a pair of collocated BPT values for the given scenario. Note that for illustrative purposes, maximum thresholds for BPTs in (A-B) are set on the y-axis and sources exceeding these thresholds are not depicted here. 


\subsection{2 $\mathrm{O}_{3}$-based Benefits-per-ton Based on a Linear CRF}

Up to this point, we have shown BPT estimates based exclusively on $\mathrm{NO}_{2}$ chronic exposure mortality. As analysis of CanCHEC suggests that a linear in concentration CRF is the most appropriate model choice for $\mathrm{O}_{3}$ (Crouse et al. 2015), we apply a linear in concentration CRF to estimate $\mathrm{O}_{3}$-based BPTs (Figure 7.6). A noticeable feature of $\mathrm{O}_{3}$ based BPT estimates at baseline 2007 emission levels (Figure 7.6A) is their wide spatial coverage. Distant sources of influence exist for $\mathrm{O}_{3}$, such as those in the northern U.S., due to its longer atmospheric lifetime and its ability to be transported over distances. At baseline, BPTs are significantly smaller than those for $\mathrm{NO}_{2}$ in Figure 7.2 due to the smaller risk coefficient (Table 7.1) and nature of $\mathrm{NO}_{2}$ to be formed closer to populous receptor regions. In some major urban cores, $\mathrm{O}_{3}$-based BPTs are negative (e.g., Vancouver, Montreal, and Toronto; Figure 7.6A). Negative BPTs, or disbenefits, have been reported before (Pappin and Hakami 2013A), and exist due to the nonlinear dependency of ground-level $\mathrm{O}_{3}$ formation on emitted precursors $\left(\mathrm{NO}_{\mathrm{x}}\right.$ and volatile organic compounds (VOCs)). In environments with a large availability of $\mathrm{NO}_{\mathrm{x}}$ compared to VOCs, $\mathrm{O}_{3}$ production is suppressed. Most densely packed urban environments currently fall into this category, as their $\mathrm{NO}_{x}$ emissions exceed those of anthropogenic and biogenic VOCs. A negative sensitivity in $\mathrm{NO}_{\mathrm{x}}$-inhibited environments indicates that any decrease in emitted $\mathrm{NO}_{x}$ would increase $\mathrm{O}_{3}$ production by reducing titration of $\mathrm{O}_{3}$ by NO. A reduction in $\mathrm{NO}_{\mathrm{x}}$ makes available more free radicals that are necessary ingredients for producing $\mathrm{O}_{3}$ and would otherwise have been scavenged by $\mathrm{NO}_{x}$. One example of a negative response of $\mathrm{O}_{3}$ to $\mathrm{NO}_{\mathrm{x}}$ is the disbenefit is Toronto, whose BPT is $-\$ 50,000 /$ ton at baseline (Figure 7.6A). 
As before, we depict BPT estimates for various large-scale, domain-wide abatement scenarios (25\% and 50\% abatement; Figure 7.6B-C and Figure 7.4B). The dominant feature of Figure 7.6 is increasing BPTs towards higher abatement levels, as in Figure 7.3 for $\mathrm{NO}_{2}$. For example, Toronto's BPT that was initially estimated at $-\$ 50,000 /$ ton for 2007 rises to $-\$ 27,000 /$ ton with $25 \%$ abatement, and eventually becomes positive, to an estimated $\$ 16,000 /$ ton with $50 \%$ abatement (Figure 7.6 and Figure 7.4B). Toronto's BPT estimates are one example of the widespread, increasing benefits with abatement observed across all source locations. As anthropogenic emissions are reduced, $\mathrm{NO}_{\mathrm{x}}$ molecules are at a higher premium, and each ton of control has an increasingly important role in mitigating $\mathrm{O}_{3}$ exposure. Similar behavior has been reported and discussed previously for the response of U.S. acute $\mathrm{O}_{3}$ exposure mortality to $\mathrm{NO}_{\mathrm{x}}$ emissions (Pappin et al. 2015A). 

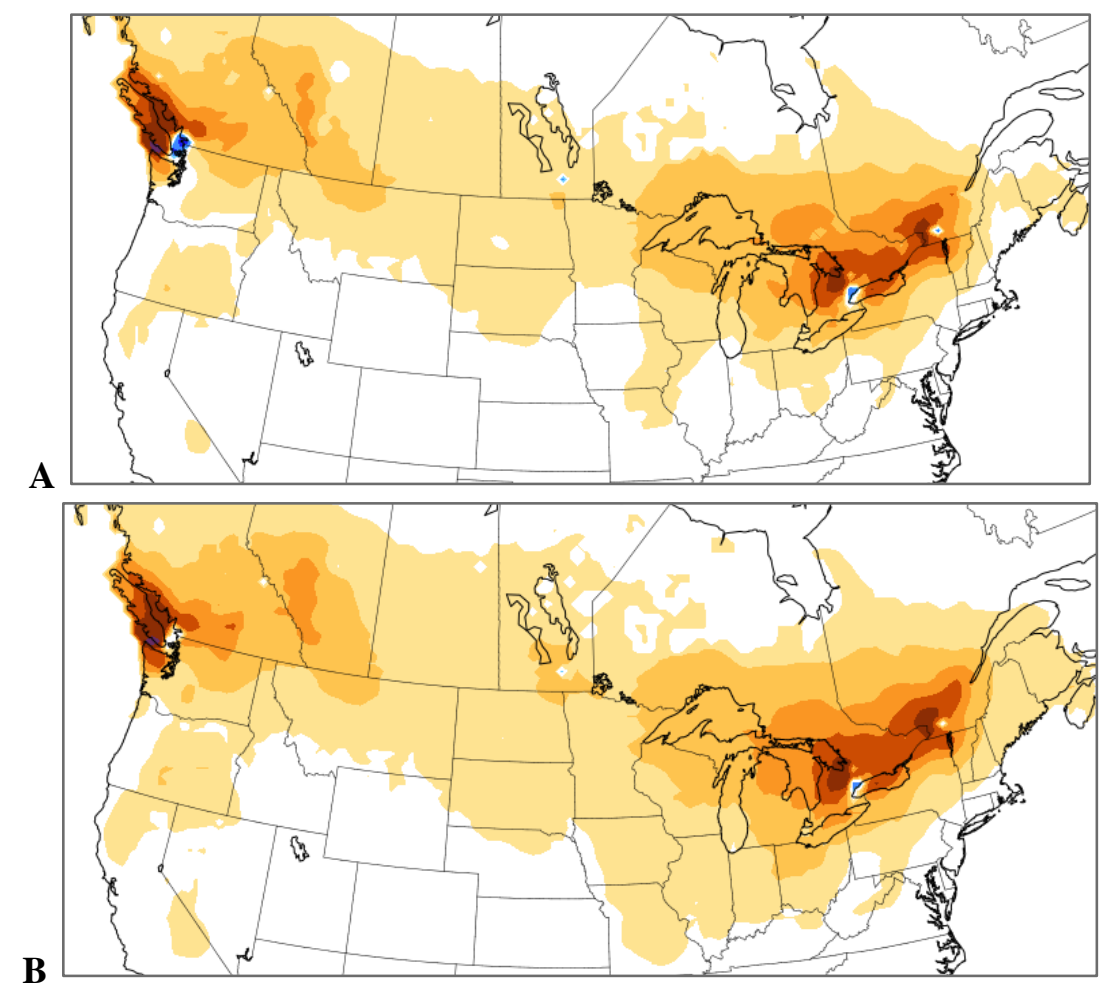

B

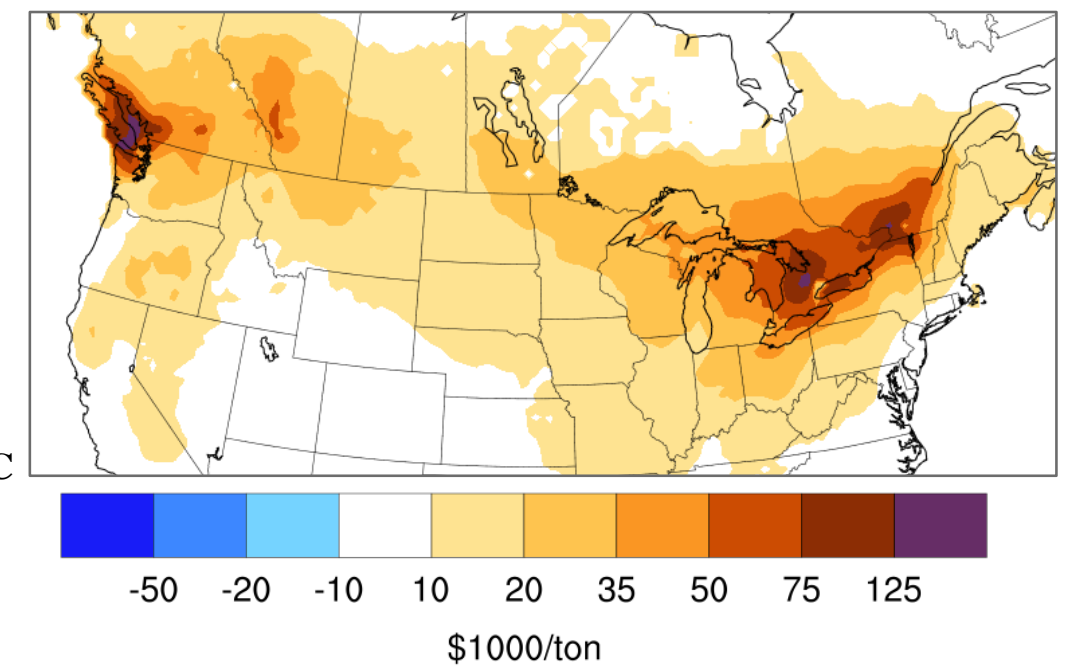

Figure 7.6 BPTs of $\mathrm{NO}_{x}$ control based on a linear in concentration CRF for $\mathrm{O}_{3}(\beta=0.0026)$ from the two-pollutant model. BPTs are estimated at (A) 2007 emission levels and for (B) $25 \%$ and (C) $50 \%$ abatement scenarios.

$\mathrm{NO}_{2}$ and $\mathrm{O}_{3}$ present two cases of increasing BPTs with abatement for two different reasons. Compounding BPTs for $\mathrm{NO}_{2}$ are incurred due to the shape of the logconcentration CRF. For $\mathrm{O}_{3}$, increasing BPTs with abatement occur entirely due to 
atmospheric chemistry. Consideration of both $\mathrm{NO}_{2}$ and $\mathrm{O}_{3}$ together (i.e., the summation of Figures 7.2 and 7.6A) would indicate compounding BPTs with continued abatement for two reasons. Regardless of the source of nonlinearity, increasing BPTs offer a new paradigm for long-term assessment of emissions abatement policies that is in contrast with the traditional view of diminishing benefits with abatement found in the environmental economics literature.

\subsubsection{Other Pollutants}

While $\mathrm{O}_{3}$ and $\mathrm{NO}_{2}$ are major criteria pollutants in ambient air associated with chronic exposure mortality in Canada, $\mathrm{NO}_{\mathrm{x}}$ emissions also contribute to formation of secondary inorganic PM. A more inclusive approach to estimating BPTs of $\mathrm{NO}_{\mathrm{x}}$ control would span over all pollutants impacted by emitted $\mathrm{NO}_{\mathrm{x}}$. Past studies have used various modeling approaches to estimate $\mathrm{PM}_{2.5}$-based BPTs in the U.S. Fann et al. (2012A) and Holt et al. (2015) found, using various applications of atmospheric CTMs, that such BPTs, or related sensitivities, increase from baseline to abatement scenarios. These findings do not exclusively apply to $\mathrm{NO}_{\mathrm{x}}$, and may extend to abatement of $\mathrm{SO}_{2}$ and even

primary PM emissions. Such findings are due to a nonlinear atmospheric response of PM to emitted precursors through aerosol thermodynamics and aqueous chemistry. With recent evidence that a supralinear CRF may be the most fitting model choice for $\mathrm{PM}_{2.5}$ (Crouse et al. 2015; Pope et al. 2009; Pope et al. 2011), added nonlinearity in health benefits with abatement is expected (Apte et al. 2015; Goodkind et al. 2014; Pope et al. 2015). Our findings for $\mathrm{NO}_{2}$ and $\mathrm{O}_{3}$ can therefore be cast in light of compounding benefits of $\mathrm{NO}_{\mathrm{x}}$ control that may remain, and even be amplified, with inclusion of $\mathrm{PM}_{2.5}$ (Pappin et al. 2015A). 


\subsection{Conclusions}

Our results are affected by a number of uncertainties introduced when integrating epidemiological risk estimates, population and mortality data, and monetary valuation metrics with atmospheric CTMs. Our results are affected by uncertainties in risk estimates for various forms of CRFs. Changes in population characteristics and mortality rates from 2007 are not captured here and may affect BPT estimates into the future, particularly as pollution levels decline with continued abatement. We apply a uniform and constant value of a statistical life to mortality in Canada, while recognizing that it may vary spatially and temporally as pollution levels decline. Uncertainty in emission inventories and in modeling complex atmospheric processes in CTMs introduces uncertainty into BPT estimates. Interpretation of our findings should consider these limitations and uncertainties of our analysis.

In estimating BPTs, we assume a causal relationship between $\mathrm{O}_{3}$ and $\mathrm{NO}_{2}$ exposure and mortality in the long-term. Our estimates of BPTs should be interpreted with a forward-looking lens and within the context of long-term public health benefits in Canada gained from emissions abatement. As exposure levels over long periods, rather than short periods, are most relevant to chronic health endpoints, the estimated benefits may take time to compound in the population. Further, our estimates are based on a reference year of 2007. Variability in BPTs is expected from year to year with changing emissions and meteorological conditions. We note that our BPT estimates for 2007 are not necessarily reflective of present-day BPTs due to the widespread and aggressive emissions abatement that has taken place since (U.S. EPA 2015). Our findings of rising 
BPTs with more aggressive emissions control suggest that BPTs for present-day emission levels are likely to exceed those for 2007 . 


\section{Chapter: $\mathrm{NO}_{\mathrm{x}}$ Control - A Gift that Keeps on Giving}

This chapter is a draft manuscript prepared under the title " $\mathrm{NO}_{\mathrm{x}}$ Control: A Gift that Keeps on Giving". ${ }^{4}$ This chapter provides perspective on a number of studies from the literature and Chapters 6-7 of this thesis that collectively support the idea that estimates of the public health benefits of reducing $\mathrm{NO}_{\mathrm{x}}$ emissions will continue to grow into the future. Amanda Pappin is the main contributor (80\%) to this chapter.

\subsection{Introduction}

Ambient air pollution is a leading risk factor for premature mortality and morbidity worldwide (Lim et al. 2012). A growing body of epidemiological evidence has demonstrated associations between short and long-term pollutant exposure and health endpoints of varying severity [Katsouyanni et al. 2009; U.S. Environmental Protection Agency (EPA) 2013]. Ozone $\left(\mathrm{O}_{3}\right)$ and fine particles $\left(\mathrm{PM}_{2.5}\right)$ are among criteria pollutants whose public health burdens are of particular interest given their high levels of exposure and severe health consequences. In the U.S., long-term exposure to $\mathrm{O}_{3}$ is estimated to lead to 19,000 deaths/year, with 320,000 deaths/year attributable to $\mathrm{PM}_{2.5}$ exposure (Fann et al. 2012B). Air pollution thus poses a substantial public health burden, even at exposure levels below current ambient air quality standards (U.S. EPA 2013).

Among various considerations in air quality decision-making, benefit-cost assessment compares desired policy implications, or benefits, with costs of pollution control. Estimating the health benefits of improvements in air quality is a nontrivial task informed by atmospheric science, public health, and economics disciplines (e.g., Hubbell

\footnotetext{
${ }^{4}$ Pappin AJ, Hakami A, Bell ML. $\mathrm{NO}_{\mathrm{x}}$ control: A gift that keeps on giving. In preparation.
} 
et al. 2005; Muller and Mendelsohn 2009; Pappin and Hakami 2013A). Quantitative assessments of such benefits have been conducted for prescribed changes in $\mathrm{O}_{3}$ concentrations (such as for attainment of standards; e.g., Hubbell et al. 2005), or for policy-driven changes in emissions (e.g., Pappin and Hakami 2013A). The latter relates emission reductions directly with societal benefits. One metric that facilitates comparison with abatement costs is the benefit-per-ton of emission control, also referred to as marginal benefit $(\mathrm{MB})$, or marginal damage $(\mathrm{MD})$, depending on perspective. $\mathrm{MB}$ is the monetary benefit of averted health and/or environmental impacts from a 1 ton reduction in emissions. In a benefit-cost assessment framework, maximizing the societal benefit of emission reductions ideally leads to a comparison of MB with the per-ton cost of control (i.e., marginal cost; MC).

Nitrogen oxides $\left(\mathrm{NO}_{\mathrm{x}}\right)$ are precursors to formation of $\mathrm{O}_{3}$ and secondary inorganic particulate matter $(\mathrm{PM})$. The $\mathrm{MB}$ of reducing $\mathrm{NO}_{\mathrm{x}}$ emissions is influenced by population exposures to components of $\mathrm{NO}_{\mathrm{x}}$ itself (i.e., $\mathrm{NO}_{2}$ ), as well as $\mathrm{O}_{3}$ and $\mathrm{PM}$. Previous studies have estimated $\mathrm{NO}_{x}$ MBs within the U.S., which differ by location and with studies employing various concentration-response functions (CRFs), economic valuation metrics, and atmospheric models with different degrees of complexity. Using advanced sensitivity analysis methods, Pappin and Hakami (2013A) estimated MBs related to $\mathrm{O}_{3}$ exposure to be as high as $\$ 75,000 /$ ton of $\mathrm{NO}_{\mathrm{x}}$ emitted at surface-level upwind of major urban centers. Pappin et al. (2015) later estimated national average $\mathrm{O}_{3}$-based $\mathrm{NO}_{\mathrm{x}} \mathrm{MBs}$ for mobile and point sources to be $\$ 13,000$ and $\$ 14,000 /$ ton. The upper range of $\mathrm{O}_{3}$-based $\mathrm{NO}_{\mathrm{x}} \mathrm{MBs}$ for eastern U.S. power plants has been estimated at about $\$ 30,000 /$ ton (Mesbah et al. 2013) to $\$ 53,000 /$ ton (Mauzerall et al. 2005). For the $\mathrm{PM}_{2.5}$ exposure pathway, Fann et al. 
(2009) used reduced-form models and found $\mathrm{NO}_{\mathrm{x}} \mathrm{MBs}$ to average $\$ 15,000 /$ ton for power plants, and $\$ 10,000 /$ ton for mobile sources among 9 urban areas of the U.S. Using a model-based source apportionment approach, Fann et al. (2012A) later estimated national-average $\mathrm{PM}_{2.5}$-based $\mathrm{NO}_{\mathrm{x}} \mathrm{MBs}$ of $\$ 3,700 /$ ton for power plants and $\$ 4,500 /$ ton for mobile sources. Studies in the environmental economics literature have often employed simplified dispersion models for estimating $\mathrm{NO}_{\mathrm{x}} \mathrm{MBs}$ that do not fully capture atmospheric nonlinearities, leading to significantly lower estimates (e.g., averaging \$260/ton; Muller and Mendelsohn 2009). Past MB estimates suggest that the largest impact of $\mathrm{NO}_{\mathrm{x}}$ emissions on health is likely through $\mathrm{O}_{3}$, though further research is necessary to support this conclusion. As $\mathrm{O}_{3}$ is potentially the dominant pathway through which $\mathrm{NO}_{\mathrm{x}}$ affects populations, it is the focus our discussion. We note that complex interactions of pollutants with dynamic factors such as climate change, that are the topic of our commentary, are often better understood for $\mathrm{O}_{3}$. Wherever possible, however, we extend our discussion to PM for comprehensiveness.

Benefit-cost assessment relies upon estimates of $\mathrm{MB}$ and MC, or total benefits and costs, for evaluating policy items. Currently, assessments often apply these measures at a static point in time and thus provide useful but limited insight into the long-term consequences of $\mathrm{NO}_{\mathrm{x}}$ control. This commentary explores aspects of the dynamic context within which benefit-cost assessments can be extended. We discuss the roles of emissions behaviour, climate change, advancing epidemiological evidence, and technological change in mitigating the health burden of $\mathrm{NO}_{\mathrm{x}}$ emissions into the future. 


\subsection{Discussion}

Tradeoffs between the MB and MC of emission reductions are important considerations for setting emission reduction targets and understanding their consequences. MB for criteria pollutants is typically shown as a positively sloped function of emissions in the environmental economics literature, while MC is negatively sloped (Figure 8.1) (Goodstein 1995; Hussen 2004). In this depiction, the benefit of reducing 1 ton of emissions is largest at highest emission level, while the cost is at its lowest (Figure 8.1). Gradual progression to lower emission levels incurs a rising MC and diminishing MB. Economic equilibrium theory suggests that emission reduction targets set at the level where MC equals $\mathrm{MB}$ are the most economically efficient $\left(\mathrm{E}^{*}\right.$ in Figure 8.1). Operating at higher levels of emissions than this incurs a net societal loss, as the incremental damage outweighs the cost savings (emissions $>E^{*}$ in Figure 8.1). On the other hand, the incremental cost below this point (emissions $<E^{*}$ in Figure 8.1) is higher than the expected benefit or return.

We note that benefit-cost assessment provides economic insight into proposed control strategies, and often accompanies the development of ambient standards for attainment or implementation policies, depending on the regulatory setting. Many other factors, however, play a role in the decision-making process, such as financial feasibility, the distribution of benefits and costs, and the views and values of stakeholders. 


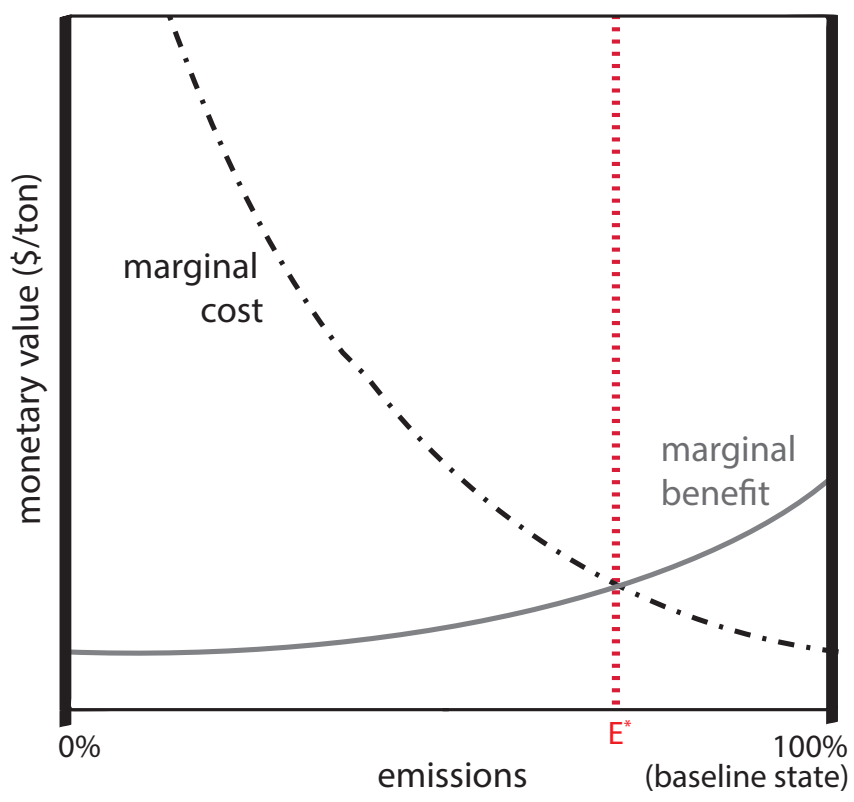

Figure 8.1 Traditional depiction of MB (solid gray line) and MC (hatched black line) as a function of emission level. The intersection of the MB and MC curves at emission level $\mathrm{E}^{*}$ (red dashed line) is the point of economic equilibrium that maximizes net societal benefits. MB and MC curves shown here are qualitative and for demonstrative purposes only.

MB estimation draws upon three nontrivial relationships: (1) the pathway from emissions to exposure (as dictated by physical and chemical atmospheric processes), (2) the epidemiological concentration-response function (CRF) for exposures and health endpoints, and (3) the monetary value applied to health endpoints. These elements collectively form the monetized health impact function:

$$
\Delta B=Y_{0} P\left(1-e^{-\beta \Delta C}\right) \cdot V A L
$$

This function monetizes the health benefit, $\Delta B$, attributable to a change in pollutant concentration, $\Delta C$, resulting from emission control. In Equation 8.1, $Y_{0}$ is the baseline health endpoint rate, $P$ is the population, and $\beta$ is the effect estimate from epidemiologic studies, which relates a unit change in concentration to a change in $Y_{0}$. The 
marginal valuation function, $V A L$, is the monetary value applied to each health endpoint (e.g., the value of statistical life) based on willingness to pay or cost-of-illness approaches. The marginal valuation function is affected by consumer preferences and budget constraints, and is theoretically dependent on the level of pollution (and hence emissions), but is considered to be constant in the context of our analysis. Equation 8.1 is one form of the health impact function used in the literature that implies a constant increased risk per unit concentration (i.e., log-transformed relative risk is linear with concentration).

\subsubsection{Marginal Benefits in the Face of Emission Reductions}

A fundamental concept in the environmental economics literature is that $\mathrm{MB}$ declines as emissions decline (Fig. 8.1). Mathematically, this is equivalent to a positive second-order derivative of the total benefit/damage function with respect to emissions, and is referred to as convexity. Convexity assumes that the atmosphere has a natural ability to cleanse itself that gradually diminishes as it becomes more polluted (Hussen 2004). While convexity, at best, may be appropriate for some primary pollutants, it fails to capture the nonlinear behaviour of secondary pollutant formation, such as that of $\mathrm{O}_{3}$ or PM from precursor species [ $\mathrm{NO}_{\mathrm{x}}$ and volatile organic compounds (VOCs)].

A well-known case that challenges the assumption of convexity for $\mathrm{O}_{3}$ lies in the negative $\mathrm{NO}_{\mathrm{x}} \mathrm{MBs}$ found in $\mathrm{NO}_{\mathrm{x}}$-rich urban areas $\left(\mathrm{NO}_{\mathrm{x}}\right.$-inhibited or VOC-limited regimes) (Fann et al. 2009; Muller and Mendelsohn 2009; Pappin and Hakami 2013A). Negative MBs indicate that a reduction in $\mathrm{NO}_{\mathrm{x}}$ would increase $\mathrm{O}_{3}$ exposure and incur a disbenefit to society. $\mathrm{NO}_{\mathrm{x}}$ is a necessary ingredient for production of $\mathrm{O}_{3}$, and in typical 
environments, $\mathrm{NO}_{\mathrm{x}}$ control is an effective control strategy. However, at high $\mathrm{NO}_{\mathrm{x}}$ concentrations (e.g., in urban cores or power plant plumes), $\mathrm{NO}_{\mathrm{x}}$ consumes radicals that are essential for $\mathrm{O}_{3}$ production, and reducing $\mathrm{NO}_{x}$ would increase $\mathrm{O}_{3}$ concentrations locally. At sufficiently low emission levels, as $\mathrm{NO}_{\mathrm{x}}$ becomes less abundant, $\mathrm{MBs}$ would eventually increase from negative to positive values. Previous studies in air pollution economics have recognized negative MBs as contradictions to the notion of convexity (e.g., Repetto 1987), but the general assumption of convexity had not been fully tested until recently. Pappin et al. (2015) recently provided evidence that convexity does not apply to $\mathrm{NO}_{\mathrm{x}}$ and $\mathrm{O}_{3}$. The authors, for the first time, quantified the $\mathrm{O}_{3}$-based $\mathrm{NO}_{\mathrm{x}} \mathrm{MB}$ curve and found, invariably and for all types of sources, that MBs increase with increasingly stringent emissions controls. In other words, each additional ton of $\mathrm{NO}_{\mathrm{x}}$ abatement would incur larger benefits than the previous ton. Rising MBs present a case for nonconvexity, or concavity, as the general rule for $\mathrm{O}_{3}$ and $\mathrm{NO}_{\mathrm{x}}$.

Beyond $\mathrm{O}_{3}$, indications of nonconvexity also exist for the response of $\mathrm{PM}$ to $\mathrm{NO}_{\mathrm{x}}$. The first indication is the work of Fann et al. (2012A), who reported consistently larger $\mathrm{PM}_{2.5}$-based MBs for $\mathrm{NO}_{\mathrm{x}}$ (and other PM precursors) for a future emission control scenario compared to 2005 levels. Recent work by Holt et al. (2015) reported a similar increase in sensitivities of $\mathrm{PM}_{2.5}$ to $\mathrm{NO}_{\mathrm{x}}$ with further emissions controls. Perhaps the most direct evidence is the prevalently negative second-order derivatives of $\mathrm{PM}$ to $\mathrm{NO}_{\mathrm{x}}$ emissions reported by Zhang et al. (2012), indicative of a concave response surface. Though MBs through PM have not been fully characterized across a spectrum of emission levels, the collective evidence suggests that nonconvexity would apply to $\mathrm{NO}_{\mathrm{x}}$ (and possibly other precursor) MBs. 
The compounding nature of MBs to increase with progressive emission controls provides added incentive to reduce emissions, even in the presence of rising MC. Depiction of MB vs. MC under this new paradigm (Fig. 2) indicates an important shift in the intersection point between MB and MC curves. The point of economic efficiency costs is likely to exist at a lower emission level than previously envisioned (Pappin et al. 2015A).

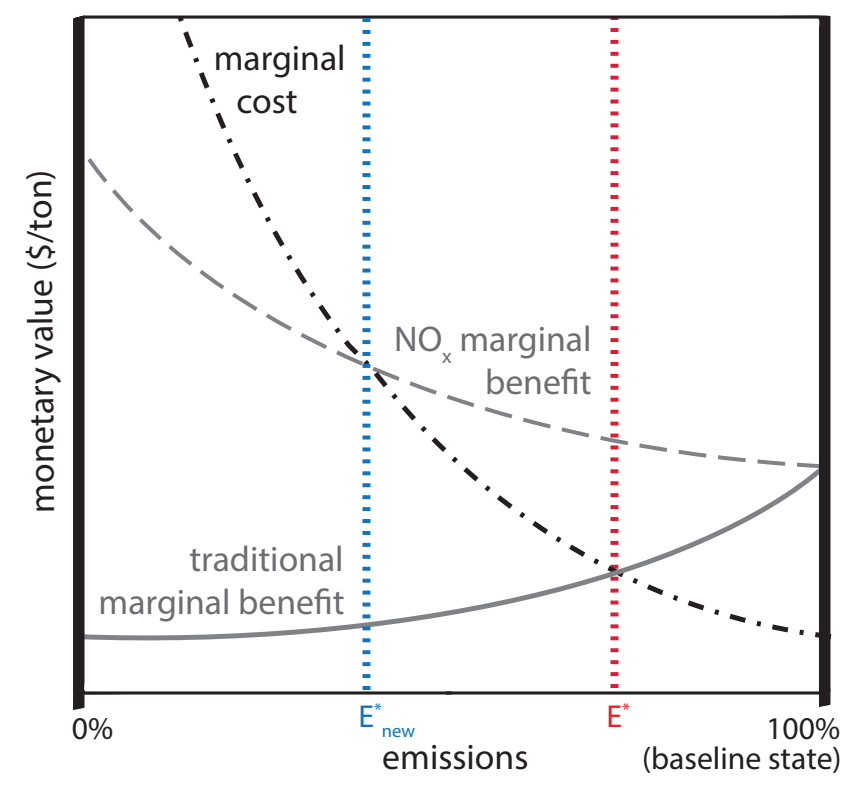

Figure 8.2 An alternative shape of the $\mathrm{NO}_{\mathrm{x}} \mathrm{MB}$ curve (dashed gray line) compared to the conventional form for criteria pollutants (solid gray line). A negative slope of MB with respect to emissions suggests that the point of economic equilibrium lies at a lower level of emissions $\left(E^{*}{ }_{\text {new }}\right.$; blue dashed line) than that suggested by traditional forms $\left(E^{*}\right.$; red dashed line $)$. We note that the slope of the MB curve depends on the combination of controls implemented across different sectors, and that the $\mathrm{NO}_{\mathrm{x}} \mathrm{MB}$ curve shown here is qualitatively based on the collective MB curve for many U.S. sources found by Pappin et al. (2015). 


\subsubsection{Marginal Benefits in the Face of a Warming Climate}

A growing body of evidence points to increased $\mathrm{O}_{3}$ concentrations, and an associated health burden, in a warming climate (Jacob and Winner, 2009). Interactions between climate and air quality therefore indicate an import area of public health vulnerability in the longer term. For example, in a 2050 climate scenario, mortality in the eastern U.S. is estimated to increase by $0.11-0.27 \%$ due to higher $\mathrm{O}_{3}$ exposure (Bell et al. 2007). Observational and model-based studies have further quantified the sensitivity of $\mathrm{O}_{3}$ to changes in temperature directly (termed climate penalty factor $\left.(\mathrm{CPF})\right)(\mathrm{Bloomer}$ et al. 2009; Doherty et al. 2013; Steiner et al. 2006; Zhao et al. 2013). Estimates of CPFs range from averages of $1.3-3.3 \mathrm{ppb} / \mathrm{K}$, regionally, to $1.34 \mathrm{ppb} / \mathrm{K}$, nationally, in the U.S. (Bloomer et al. 2009; Zhao et al. 2013). This translates into an estimated health impact of 370 deaths/K increase in temperature across North America (Zhao et al. 2013).

Past studies have noted that the most prominent attribute of $\mathrm{CPF}$ is its spatial heterogeneity. Using adjoint sensitivity analysis, Zhao et al. (2013) found CPFs in urban areas to be consistently larger than those of less polluted environments, exceeding 6.2 $\mathrm{ppb} / \mathrm{K}$ in some places. In general, rising temperatures affect $\mathrm{O}_{3}$ through (1) increased atmospheric reaction rates, (2) higher availability of atmospheric water vapor, and (3) increased biogenic VOC emissions. Urban $\mathrm{O}_{3}$ climate vulnerability occurs because in a $\mathrm{NO}_{\mathrm{x}}$-rich environment, $\mathrm{O}_{3}$ production is exacerbated by any increase in availability of free radicals. Increased biogenic VOC emissions with rising temperatures thus facilitate urban $\mathrm{O}_{3}$ production. Further, increased atmospheric water vapor with rising urban temperatures would remove $\mathrm{O}_{3}$ in most environments, but instead increases $\mathrm{O}_{3}$ production in urban areas by making available radicals that initiate its photochemical production. 
This duality of behaviour is dependent on $\mathrm{NO}_{\mathrm{x}}$ availability and is the main source of spatial variability in CPFs.

The general finding of larger CPFs in cities indicates an urban $\mathrm{O}_{3}$ vulnerability to climate change. $\mathrm{NO}_{\mathrm{x}}$ control may mitigate this sensitivity, yielding indirect and longerterm health benefits. This conclusion by Zhao et al (2013) is also manifested in the work of Bloomer et al. (2009), who observed a decline in CPFs from 3.2 to $2.2 \mathrm{ppb} / \mathrm{K}$, on average, in the eastern U.S. over 1987-2007. The authors attributed this decline to regional reductions in $\mathrm{NO}_{\mathrm{x}}$ emissions from power plants that averaged $43 \%$ pre-2002 to post-2002. Progressive $\mathrm{NO}_{\mathrm{x}}$ emission reductions may therefore partially compensate for the impact of rising temperatures on $\mathrm{O}_{3}$ levels by reducing the sensitivity of $\mathrm{O}_{3}$ health impacts to climate-induced warming. In other words, $\mathrm{NO}_{\mathrm{x}}$ control can be regarded as a climate change adaptation strategy in the long-term.

We note that there are various, complex interactions between climate and air quality that we do not discuss here. As an example, $\mathrm{NO}_{\mathrm{x}}$ control has an impact on climate itself through methane (Fry et al. 2012; Wild et al. 2001), which in turn has feedback on $\mathrm{O}_{3}$ production in the long-term. The indirect benefit of $\mathrm{NO}_{\mathrm{x}}$ control through reduced climate penalty is one unexplored pathway in benefit-cost assessment.

\subsubsection{The Role of Advancing Epidemiological Evidence in Marginal Benefit}

\section{Estimation}

Estimation of $\mathrm{MB}$ is dependent on the state of epidemiological evidence documenting associations between pollutant exposures and health endpoints. In many cases, advancing epidemiological evidence points to excess health risks for endpoints not 
previously considered in benefit-cost assessment, or otherwise confirms suspected associations. The association between $\mathrm{O}_{3}$ and mortality (a primary contributor to $\mathrm{MB}$ because of its high monetary value) is a prime example. In 1996, the U.S. EPA could not quantify an $\mathrm{O}_{3}$-mortality risk estimate or even assess the likelihood of an $\mathrm{O}_{3}$-mortality association based on the epidemiological evidence of the time. Later, in 2006, the EPA determined that the collective evidence was "highly suggestive" of an effect, and in the most recent assessment, that the association between short-term exposure to $\mathrm{O}_{3}$ and mortality was robustly demonstrated in the literature (U.S. EPA 2013).

Advancing knowledge of excess health risks, particularly at low ranges of exposure levels, has important implications for the MB curve. Current evidence does not support the theory of an exposure threshold for $\mathrm{O}_{3}$ or $\mathrm{PM}_{2.5}$ below which health is not impacted (Bell et al. 2006; Daniels et al. 2000). In fact, recent studies have suggested the opposite for some pollutants - that the increased risk per unit concentration is highest at low levels of exposure, and diminishes as the environment becomes more polluted. While not directly applicable to $\mathrm{O}_{3}$, such a supralinear form of the CRF indicates a heightened sensitivity of populations in cleaner environments to any changes in exposure to $\mathrm{PM}_{2.5}$ and $\mathrm{NO}_{2}$ (Crouse et al. 2015; Pope et al. 2011). A supralinear CRF would result in rising MBs, and accrued public health benefits, with progressive emission controls (Goodkind et al. 2015; Pope et al. 2015).

Application of CRFs for MB estimation is also affected by temporal changes in underlying population characteristics. These may include changes in baseline health endpoint rates, the distribution of sensitive subpopulations (e.g., a higher proportion of older or minority persons), and trends in adaptive measures (e.g., increasing prevalence 
of central air conditioning that attenuates risk) over time. Trends in population characteristics, in addition to advancing epidemiological evidence, thus form a dynamic context for characterizing the benefits of emission control, and have important policy implications.

\subsubsection{Marginal Costs in the Face of Technological Advancement and Learning by Doing}

We note that assessments of MC routinely account for the general decline in purchasing power that occurs with inflation. This is not a topic of discussion here, as inflation would similarly act on MB estimates and thus not change the overall recommendations of benefit-cost assessments. On the other hand, economic growth and research and development are important factors that solely affect the $\mathrm{MC}$ of $\mathrm{NO}_{\mathrm{x}}$ control. Such factors are challenging to predict, but may be important for economic assessments extending far into the future, particularly as costs rise with more stringent emission reductions. One example of cost savings is that offered by "learning by doing". This concept refers to the increase in production and efficiency as experience with a given emission control technology increases (U.S. EPA 2011). In a recent report assessing the benefits and costs of the Clean Air Act and associated Amendments, the U.S. EPA (2011) projected compliance cost reductions of $10 \%$ by 2020 through the mechanism of learning by doing. In addition to learning by doing, technological change can offset the rising costs of increasingly stringent emission controls. Economic studies that model the effects of technological change on abatement costs often assume that, overall, costs decline with research and development (Baker et al. 2008). A relevant example for $\mathrm{NO}_{\mathrm{x}}$ control is

provided by Isakkson (2005). From 1991-1996, $\mathrm{NO}_{\mathrm{x}}$ emissions in the Swedish energy 
sector were reduced by $35 \%$ through an emissions tax that induced a period of rapid technological change. Over that time, the sector-wide abatement cost curve shifted downwards, by up to $50 \%$. This shift was attributed in part to the rapid technological change over that time period.

We note that shifts to new technologies may only be feasible initially, depending on the combination of fixed and variable costs of a given option. Alternatively, new control options may only be feasible for large-scale reductions in emissions, such as for technological shifts requiring large infrastructure or more fundamental changes in human behaviours. These factors are complex and uncertain, but past trends indicate important reductions in cost that may extend into the future.

\subsection{Conclusions}

We do not account for a number of other dynamic factors that have potential to influence benefit-cost assessment. These include changing emission behaviours under climate change, shifts in reliance on natural resources, advances in economic valuation measures, and factors affecting the world economy. Our evaluation is based on the use of atmospheric models that undergo flux as the scientific understanding of atmospheric processes matures. Similarly, the scientific underpinnings of health effects estimation and monetary valuation methods continuously evolve. Our analysis focuses on the impacts of $\mathrm{NO}_{\mathrm{x}}$ emission reductions on $\mathrm{O}_{3}$-related health benefits, with less consideration for effects related to other species, such as $\mathrm{NO}_{2}$ and inorganic PM. Further research is required to assess MB in a multi-pollutant framework. 
We maintain that $\mathrm{NO}_{\mathrm{x}}$ emission controls have indirect and sustained benefits in a future world beyond their immediate health impacts. Aspects of the dynamic nature of benefit-cost assessment discussed here add to the existing weight of evidence in support of more stringent $\mathrm{NO}_{\mathrm{x}}$ abatement. 


\section{Chapter: Conclusion}

The research presented in this thesis has contributed to the field of air quality modelling through the development of a novel method to conduct health impact assessment using advanced sensitivity analysis. It has explored different angles and perspectives on questions of practical importance to air quality management, as described in Chapter 1 and summarized below,

- What are the spatial and temporal behaviours of health metrics attributed to emissions?

- How do behaviours differ between health metrics and metrics based on attainment of standards?

- How do attributed health impacts/benefits respond to dynamic changes brought by progressive environmental policies?

- How does our understanding of epidemiology affect our estimates of source attributed health benefits?

- What do these findings mean for air quality and health policy?

Attributing public health impacts to sources of emissions using adjoint sensitivity analysis - a method developed in Chapter 4 and applied in Chapters 5-7 - provides information for evidence-based decision-making. The results presented in these chapters show that there is substantial variability in the public health impacts of emissions, and hence in the benefits of their control, both from location-to-location (Chapters 4-7) and day-to-day (Chapters 4-5). 
This thesis provides insight on how different objectives of air quality management can be best achieved through quantifying levels of effectiveness for various strategies (Chapter 5). The results of Chapter 5 indicate potential for aligning strategies that prioritize either regulatory standards attainment or public health. A combined approach that selectively targets emission sources that exert large influences on both standards attainment and population exposure would yield benefits on both policy fronts.

Chapters 6 and 7 of this thesis provide evidence that the long-held economic theory of diminishing returns does not apply to the management of ozone and $\mathrm{NO}_{2}$ air pollution. They explore the atmospheric science and epidemiological reasons for compounding benefits of $\mathrm{NO}_{\mathrm{x}}$ emissions abatement. For ozone, compounding benefits exist solely due to atmospheric chemistry (Chapters 6-7). For $\mathrm{NO}_{2}$, the atmospheric response is linear, but recent indications of a supralinear CRF would yield rising benefits of further $\mathrm{NO}_{\mathrm{x}}$ control (Chapter 7).

A number of factors describing the social, economic, scientific, and technological environments are relevant to quantifying the benefits of emissions control for long-term decision-making (Chapter 8). These factors collectively suggest that the benefits of control increase over time, and as scientific evidence yields more refined information on the risks of air pollution exposure.

The findings presented in this thesis should be considered in the context of a number of limitations of the analyses. The modelling in Chapters 4-7 of this thesis was conducted at a coarse spatial resolution $(36 \mathrm{~km})$ and for a short simulation period (summertime) from 2007. Fine spatial gradients in emissions and population exposure, 
and hence in source-attributed health benefits, are not well represented within cities and suburban areas. More local analyses at higher resolutions that incorporate region-specific effect estimates, valuation measures, and age-specific population characteristics would yield more refined information on health benefits. Longer simulation episodes, specifically those that span over a full year to a few years, are necessary to examine the seasonal and inter-annual variability in source-attributed health benefits for long-term decision-making. National emissions trends indicate aggressive $\mathrm{NO}_{\mathrm{x}}$ emission reductions prior to and since 2007 (ECCC 2014; U.S. EPA 2015). Modelling of more recent calendar years is therefore necessary for source-attributed health benefits to better reflect the current composition of the atmosphere before directly informing policy. More quantitative analyses of marginal benefit and cost curves would further support the conclusions made in Chapters 6 and 8 and help pinpoint a shift in the economic efficiency point under the new paradigm of compounding benefits.

The spatial and temporal variability found in source-attributed health benefits in this thesis (Chapters 4-5) can be exploited for air quality management. Targeted emissions control policies can be devised that selectively control sources (1) whose emissions are most damaging, and (2) in a timely manner for short-term, adaptive control policies.

Health impact assessment relies upon measures of daily exposure for estimating acute health effects and long-term average exposure for estimating chronic health effects. In this way, benefit-cost approaches to air quality management rely on shifting the average of the exposure distribution for reducing the public health burden of air pollution. Regulatory standards, on the other hand, are set for annual average concentrations (for 
$\mathrm{PM}_{2.5}$ ) or for extreme values (for ozone). The latter approach to regulating ground-level ozone dictates a focus on mitigating extreme pollution episodes instead of the entire distribution of concentrations. While policies to improve attainment of regulatory standards for ozone could result in an overall shift in the distribution, the current objective of these policies is to mitigate extreme values. Quantification of the response of various policy metrics to abatement measures (Chapter 5) can help coordinate objectives of various regulatory, policy, or economic instruments that may not be aligned.

Non-convexity of the damage function found in this thesis (Chapters 6-7) has important policy implications. The new, supralinear form of CRF and nonlinear ozone production chemistry indicate that the benefits of emissions control in cleaner environments are larger than previously understood. The nature of emission control benefits to rise with further abatement efforts points to an economically efficient target level of emission reduction that is further down the abatement trajectory. We are currently at an important point on the abatement trajectory, where the benefits of emission controls have increased considerably in the past and will do so in the near future with continued emissions control policies. Regard for the compounding nature of emission control benefits can cast abatement policies in a self-propagating, selfrewarding light in the long-term.

A number of research topics would complement the work of this thesis and provide insight on questions that still remain unanswered or unexplored in this field. Recommendations for future research stemming from or related to this work are highlighted below. 
The notion of increasing ozone and $\mathrm{NO}_{2}$-related health benefits with progressive emissions abatement steps (Chapters 6-7) is of broad policy relevance, but it remains unclear how $\mathrm{PM}_{2.5}$ exposure would respond to large-scale and progressive reductions in primary and precursor emissions along the policy trajectory. Future research efforts that quantitatively explore how the $\mathrm{PM}_{2.5}$-related health benefits of emissions control change as the environment becomes cleaner would provide a greater understanding of the multipollutant context within which criteria pollutants respond to changes in human behaviour and consequently affect public health. Applications of sensitivity analysis that combine both atmospheric science and epidemiological perspectives in answering this question (as in Chapter 7) are of greatest relevance.

Source attributed health benefits presented in Chapters 4-7 of this thesis are based on a regional modelling domain with a $36-\mathrm{km}$ horizontal resolution. At this scale, fine gradients in emissions and consequent exposures that exist in densely populated urban and suburban areas are not well represented. Future work examining how health benefit estimates change with finer grid resolutions would yield invaluable information on how emission influences carry to resolutions within a city. Further, this thesis is based on a single simulation episode of short duration (summer for ozone and $\mathrm{NO}_{2}$ ). Future work examining the inter-annual variability in health benefits for all pollutants, and particularly the inter-seasonal variability for $\mathrm{PM}_{2.5}$ and $\mathrm{NO}_{2}$, would provide insight on the duration of simulation episodes necessary for informing evidence-based decision-making in the longterm.

In addition to the above-mentioned areas of future research, exploiting sectoral health risk information for use in air quality planning remains an evolving area. Future 
work that examines the unique signatures of specific PM source sectors and their public health impacts would yield insight on the effectiveness of sectoral emission control programs. Further estimation of marginal benefit curves for such source sectors would aid in understanding the individual roles that source sectors play in the long-term. The compounding nature of benefits and nonconvexity of damage functions suggest synergistic benefits from sectoral control (see Chapter 6). Efforts to quantitatively evaluate these benefits should be mindful of the regional and global context within which sectoral policies take effect. 


\section{Bibliography}

Alberini A, Hunt A, Markandya A. 2006. Willingness to pay to reduce mortality risks: evidence from a three-country contingent valuation study. Environ Resour Econ $33: 251-264$

Anderson S, Francois P. 1997. Environmental cleanliness as a public good: welfare and policy implications of nonconvex preferences. J Environ Econ Manage 34:256274.

Anenberg SC, Horowitz LW, Tong DQ, West JJ. 2010. An estimate of the global burden of anthropogenic ozone and fine particulate matter on premature human mortality using atmospheric modeling. Environ Health Perspect 118:1189-1195.

Anenberg SC, West JJ, Fiore AM, Jaffe DA, Prather MJ, Bergmann D, et al. 2009. Intercontinental impacts of ozone pollution on human mortality. Environ Sci Technol 43:6482-6487.

Apte JS, Marshall JD, Brauer M, Cohen AJ. 2015. Addressing global mortality from ambient $\mathrm{PM}_{2.5}$. Environ Sci Technol 49:8057-8066.

Banzhaf HS, Burtraw D, Palmer K. 2004. Efficient emission fees in the US electricity sector. Resour Energy Econ 26:317-341.

Baker E, Clarke L, Shittu E. 2008. Technical change and the marginal cost of abatement. Energy Econ 30:2799-2816.

Barrett SRH, Yim SHL, Gilmore CK, Murray LT, Kuhn SR, et al. 2012. Public health, climate, and economic impacts of desulfurizing jet fuel. Environ Sci Technol $46: 4275-4282$ 
Baumol WJ, Bradford DF. 1972. Detrimental externalities and non-convexity of the production set. Economica 39:160-176.

Bell ML, Dominici F. 2008. Effect modification by community characteristics on the short-term effects of ozone exposure and mortality in 98 US communities. Am J Epidemiol 167:986-997.

Bell ML, Dominici F, Ebisu K, Zeger SL, Samet JM. 2007. Spatial and temporal variation in $\mathrm{PM}_{2.5}$ chemical composition in the United States for health effects studies. Environ Health Perspect 115:989-995.

Bell ML, Dominici F, Samet JM. 2005. A meta-analysis of time-series studies of ozone and mortality with comparison to the national morbidity, mortality, and air pollution study. Epidemiol 16:436-445.

Bell ML, Hobbs BF, Ellis H. 2005. Metrics matter: conflicting air quality rankings from different indices of air pollution. J Air Waste Manag Assoc 55:97-106.

Bell ML, McDermott A, Zeger SL, Samet JM, Dominici F. 2004. Ozone and short-term mortality in 95 US urban communities, 1987-2000. JAMA 292:2372-2378.

Bell ML, Peng RD, Dominici F. 2006. The exposure-response curve for ozone and risk of mortality and the adequacy of current ozone regulations. Environ Health Perspect 114:532-536.

Bergin MS, West JJ, Keating TJ, Russell AG. 2005. Regional atmospheric pollution and transboundary air quality management. Ann Rev Environ Resour 30:1-37. 
Berman JD, Fann N, Hollingsworth JW, Pinkerton KE, Rom WN, et al. 2012. Health benefits from large-scale ozone reduction in the United States. Environ Health Perspect 120:1404-1410.

Bloomer BJ, Stehr JW, Piety CA, Salawitch RJ. 2009. Observed relationships of ozone air pollution with temperature and emissions. Geophys Res Lett 36:L09803.

Brauer M, Amann M, Burnett RT, Cohen A, Dentener F, et al. 2012. Exposure assessment for estimation of the global burden of disease attributable to outdoor air pollution. Environ Sci Technol 46:652-660.

Brook JR, Burnett RT, Dann TF, Cakmak S, Goldberg MS, Fan X, et al. 2007. Further interpretation of the acute effect of nitrogen dioxide observed in Canadian timeseries studies. J Expo Sci Environ Epidemiol 17:S36-S44.

Burnett RT, Brook J, Dan T, Delocla C, Philips O, et al. 2000. Association between particulate- and gas-phase components of urban air pollution and daily mortality in eight Canadian cities. Inhalation Toxicol 12:15-39.

Burnett RT, Cakmak S, Brook JR. 1998. The effect of the urban ambient air pollution mix on daily mortality rates in 11 Canadian cities. Can J Public Health 89:152156.

Burnett RT, Goldberg MS. 2003. Size-fractionated particulate mass and daily mortality in eight Canadian cities. In Special Report: Revised Analyses of Time-Series Studies of Air Pollution and Health, Part II. Boston, MA:Health Effects Institute. 
Burnett RT, Pope III CA, Ezzati M, Olives C, Lim SS, et al. 2014. An integrated risk function for estimating the global burden of disease attributable to ambient fine particulate matter exposure. Environ Health Perspect 122:397-403.

Burnett RT, Stieb D, Brook JR, Cakmak S, Dales R, et al. 2004. Associations between short-term changes in nitrogen dioxide and mortality in Canadian cities. Arch Environ Health 59:228-236.

Byun D, Schere KL. 2006. Review of the governing equations, computational algorithms, and other components of the Models-3 Community Multiscale Air Quality (CMAQ) modeling system. Appl Mech Rev 59:51-77.

CCME (Canadian Council of Ministers of the Environment). 2000. Canada-wide Standards for Particulate Matter (PM) and Ozone. Quebec City, QC:Canadian Council of Ministers of the Environment.

CCME (Canadian Council of Ministers of the Environment). 2007. Guidance Document on Achievement Determination: Canada-Wide Standards for Particulate Matter and Ozone. Winnipeg, MB:Canadian Council of Ministers of the Environment.

CCME (Canadian Council of Ministers of the Environment). 2010. Comprehensive Air Management System: A Proposed Framework to Improve Air Quality Management. Winnipeg, MB: Canadian Council of Ministers of The Environment.

CMA (Canadian Medical Association). 2008. No Breathing Room: National Illness Costs of Air Pollution. Canadian Medical Association. Available: 
http://www.healthyenvironmentforkids.ca/resources/no-breathing-room-costs-ofair-pollution [accessed 1 January 2016].

Carter WPL. 2000. Documentation of the SAPRC99 Chemical Mechanism for VOC Reactivity Assessment. Riverside, CA:University of California.

Chestnut LG, De Civita P. 2009. Economic Valuation of Mortality Risk Reduction: Review and Recommendations for Policy and Regulatory Analysis.

Cohan DS, Hakami A, Hu Y, Russell AG. 2005. Nonlinear response of ozone to emissions: source apportionment and sensitivity analysis. Environ Sci Technol 39:6739-6748.

Cohan DS, Hu Y, Russel AG. 2006. Dependence of ozone sensitivity analysis on grid resolution. Atmos Environ 1994:126-135.

Cohan DS, Tian D, Hu Y, Russell AG. 2006. Control strategy optimization for attainment and exposure mitigation: case study for ozone in Macon, Georgia. Environ Manag $38: 451-462$.

Crocker TD, Forster B. 1981. Decision problems in the control of acid precipitation: nonconvexities and irreversibilities. J Air Pollut Control Assoc 31:31-37.

Crouse DL, Peters PA, van Donkelaar A, Goldberg MS, Villeneuve PJ, et al. 2012. Risk of nonaccidental and cardiovascular mortality in relation to long-term exposure to low concentrations of fine particulate matter: a Canadian national-level cohort study. Environ Health Perspect 120:708-714.

Crouse DL, Peters PA, Hystad P, Brook JR, van Donkelaar A, et al. 2015. Ambient $\mathrm{PM}_{2.5}, \mathrm{O}_{3}$, and $\mathrm{NO}_{2}$ exposures and associations with mortality over 16 years of 
follow-up in the Canadian Census Health and Environment Cohort (CanCHEC). Environ Health Perspect 123:1180-1186.

Daniels MJ, Dominici F, Samet JM, Zeger SL. 2000. Estimating particulate mattermortality dose-response curves and threshold levels: an analysis of daily timeseries for the 20 largest US cities. Am J Epidemiol 152:397-406.

Doherty RM, Wild O, Shindell DT, Zeng G, MacKenzie IA, et al. 2013, Impacts of climate change on surface ozone and intercontinental ozone pollution-a multimodel study. J Geophys Res Atmos 118:3744-3763.

Dominici F, McDermott A, Daniels M, Zeger SL, Samet JM. 2003. Mortality Among Residents of 90 Cities. In Special Report: Revised Analyses of Time-Series Studies of Air Pollution and Health. Boston, MA:Health Effects Institute.

Dominici F, Peng RD, Zeger SL, White R H, Samet JM. 2007. Particulate Air Pollution and Mortality in the United States: Have the Risks Changed from 1987 to 2000? Am J Epidemiol 166:880-888.

Dunker AM. 1981. Efficient calculation of sensitivity coefficients for complex atmospheric models. Atmos Environ 15:1155-1161.

Elbern H, Schmidt H, Talagrand O, Ebel A. 2000. 4D-variational data assimilation with an adjoint air quality model for emission analysis. Environ Model Softw 15:539548.

ECCC (Environment and Climate Change Canada). 2014. Air Pollutant Emission Inventory Report 1990-2014. En81-26E-PDF. Gatineau, QC:Environment and Climate Change Canada. 
Fann N, Baker KR, Fulcher CM. 2012A. Characterizing the $\mathrm{PM}_{2.5}$-related health benefits of emission reductions for 17 industrial, area and mobile emission sectors across the U.S. Environ Int 49:141-151.

Fann N, Fulcher CM, Hubbell BJ. 2009. The influence of location, source, and emission type in estimates of the human health benefits of reducing a ton of air pollution. Air Qual Atmos Health 2:169-176.

Fann N, Lamson AD, Anenberg SC, Wesson K, Risley D, et al. 2012B. Estimating the national public health burden associated with exposure to ambient $\mathrm{PM}_{2.5}$ and ozone. Risk Analy 32:81-95.

Fann NL, Phillips SB, Jang C, Akhtar FH. 2011. Comment on "Do some $\mathrm{NO}_{\mathrm{x}}$ emissions have negative environmental damages? Evidence and implications for policy". Environ Sci Technol 45:10290-10290.

Fang Y, Naik V, Horowitz LW, Mauzerall DL. 2013. Air pollution and associated human mortality: the role of air pollutant emissions, climate change and methane concentration increases from the preindustrial period to present. Atmos Chem Phys 13:1377-1394.

Farhat N, Ramsay T, Jerrett M, Krewski D. 2013. Short-term effects of ozone and $\mathrm{PM}_{2.5}$ on mortality in 12 Canadian cities. J Environ Protect 4:18-32.

Fraas A, Lutter R. 2011. Do some $\mathrm{NO}_{\mathrm{x}}$ emissions have negative environmental damages? Evidence and implications for policy. Environ Sci Technol 45:7613-7614.

Fraas A, Lutter R. 2012. Comment on "Efficient pollution regulation: getting the prices right”. Am Econ Rev 102:602-607. 
Fry MM, Naik V, West JJ, Schwarzkopf MD, Fiore AM, at al. 2012. The influence of ozone precursor emissions from four world regions on tropospheric composition and radiative climate forcing. J Geophys Res 117:D07306.

Goodkind AL, Coggins JS, Marshall JD. 2014. A spatial model of air pollution: the impact of the concentration-response function. J Assoc Environ Res Econ 1:451479.

Goodstein ES. 1995. Economics and the Environment. Englewood Cliffs, NJ:PrenticHall, Inc.

Hakami A, Henze DK, Seinfeld JH, Singh K, Sandu A, et al. 2007. The adjoint of CMAQ. Environ Sci Technol 41:7807-7817.

Hakami A, Odman MT, Russell AG. 2003. High-order, direct sensitivity analysis of multidimensional air quality models. Environ Sci Technol 37:2442-2452.

Hakami A, Odman MT, Russell AG. 2004. Nonlinearity in atmospheric response: a direct sensitivity analysis approach. J Geophys Res 109:D15303.

Hakami A, Seinfeld JH, Chai T, Tang Y, Carmichael GR, et al. 2006. Adjoint sensitivity analysis of ozone nonattainment over the continental United States. Environ Sci Technol 40:3855-3864.

Hall DC, Hall JV. 1997. Estimating the benefits of emissions reductions in complex atmospheres. Int J Glob Energy Issues 9:286-298.

Holt J, Selin NE, Solomon S. 2015. Changes in inorganic fine particulate matter sensitivities to precursors due to large-scale US emissions reductions. Environ Sci Technol 49:4834-4841. 
Health Canada. 2016. Human Health Risk Assessment for Ambient Nitrogen Dioxide. H144-31/2016E-PDF. Ottawa, ON:Health Canada.

Hussen AM. 2004. Principles of Environmental Economics. $2^{\text {nd }}$ ed. New York, NY:Routledge.

HEI (Health Effects Institute). 2002. Understanding the Health Effects of Components of the Particulate Matter Mix: Progress and Next Steps. Cambridge, MA:Health Effects Institute. Available: http://pubs.healtheffects .org/getfile.php?u=244 [accessed 12 June 2016].

Hogrefe C, Rao ST. 2001A. Demonstrating attainment of air quality standards: integration of observations and model predictions into the probabilistic framework. J Air Waste Manag Assoc 51:1060-1072.

Hogrefe C, Rao ST, Kasibhatla P, Hao W, Sistla G, et al. 2001B. Evaluating the performance of regional-scale photochemical modeling systems: part II - ozone predictions. Atmos Environ 35:4175-4188.

Hubbell BJ. 2006. Implementing QALYs in the analysis of air pollution regulations. Environ Res Econ 34:365-384.

Hubbell BJ, Hallberg A, McCubbin DR, Post E. 2005. Health-related benefits of attaining the 8-hr ozone standard. Environ Health Perspect 113:73-82.

Hystad P, Setton E, Cervantes A, Poplawski K, Deschenes S, et al. 2011 Creating national air pollution models for population exposure assessment in Canada. Environ Health Perspect 119:1123-1129. 
Ito K, De Leon SF, Lippmann M. 2005. Associations between ozone and daily mortality: analysis and meta-analysis. Epidemiol 16:446-457.

The Institute for the Environment. 2013. SMOKE v3.5.1 User's Manual. Chapel Hill, NC:The University of North Carolina.

Isakkson LH. 2005. Abatement costs in response to the Swedish charge on nitrogen oxide emissions. J Environ Econ and Manag 50:102-120.

Ito K, De Leon SF, Lippmann M. 2005. Associations between ozone and daily mortality: analysis and meta-analysis. Epidemiol 16:446-457.

Jacob DJ. 2007. Models of Atmospheric Transport and Chemistry. Chapter 1: The Continuity Equation. Available: http://acmg.seas.harvard.edu/education.html\#graduatem [accessed 19 November 2015].

Jacob DJ, Winner DA. 2009. Effect of climate change on air quality. Atmos Environ 43:51-63.

Jerrett M, Burnett RT, Pope CA III, Ito K, Thurston G, et al. 2009. Long-term ozone exposure and mortality. N Engl J Med 360:1085-95.

Judek S, Stieb D, Jovic B. 2006. Air Quality Benefits Assessment Tool (AQBAT) release 1.0. Ottawa:Health Canada.

Katsouyanni K, Samet JM, Anderson HR, Atkinson R, Le Tertre A, et al. 2009. Air Pollution and Health: A European and North American Approach (APHENA). Boston, MA:Health Effects Institute. 
Koo J, Wang Q, Henze DK, Waitz IA, Barrett SRH. 2013. Spatial sensitivities of human health risk to intercontinental and high-altitude pollution. Atmos Environ 71:140147.

Krall JR, Anderson GB, Dominici F, Bell ML, Peng RD. 2013. Short-term exposure to particulate matter constituents and mortality in a national study of U.S. urban communities. Environ Health Perspect 121:1148-1153.

Krewski D, Jerrett M, Burnett RT, Ma R, Hughes E, et al. 2009. Extended Follow-up and Spatial Analysis of the American Cancer Society Study Linking Particulate Air Pollution and Mortality. Boston, MA:Health Effects Institute.

Latza U, Gerdes S, Baur X. 2009. Effects of nitrogen dioxide on human health: systematic review of experimental and epidemiological studies conducted between 2002 and 2006. Int J Hyg Environ Health 212:271-287.

Lee CJ, Martin RV, Henze DK, Brauer M, Cohen A, et al. 2015. Response of global particulate-matter-related mortality to changes in local precursor emissions. Environ Sci Technol 49:4335-4344.

Levy J, Chemerynski S, Sarnat J. 2005. Ozone exposure and mortality: an empiric Bayes metaregression analysis. Epidemiol 16:458-468.

Lim SS, Vos T, Flazman AD, Danaei G, Shibuya K, et al. 2012. A comparative risk assessment of burden of disease and injury attributable to 67 risk factors and risk factor clusters in 21 regions, 1990-2010: a systematic analysis for the Global Burden of Disease Study 2010. Lancet 380:2224-2260. 
Mauzerall DL, Sultan B, Kim N, Bradford DF. 2005. $\mathrm{NO}_{\mathrm{x}}$ emissions from large point sources: variability in ozone production, resulting health damages and economic costs. Atmos Environ 39:2851-2866.

Mesbah SM, Hakami A, Schott S. 2013. Optimal ozone reduction policy design using adjoint-based $\mathrm{NO}_{\mathrm{x}}$ marginal damage information. Environ Sci Technol 47:1352813535.

Mendelsohn R Muller NZ. 2015. Comment on "Diminishing returns or compounding benefits of air pollution control? The case of $\mathrm{NO}_{\mathrm{x}}$ and ozone". Environ Sci Technol 50:500-501.

Montgomery WD. 1972. Market in licences and efficient pollution control program. J Econ Theory 5:395-418.

Moolgavkar SH, McClellan RO, Dewanji A, Turim J, Luebeck EG, et al. 2013. Timeseries analyses of air pollution and mortality in the United States: a subsampling approach. Environ Health Perspect 121:73-78.

Muller NZ, Mendelsohn R. 2007. Measuring the damages of air pollution in the United States. J Environ Econ Manage 54:1-14.

Muller NZ, Mendelsohn R. 2009. Efficient pollution regulation: getting the prices right. Am Econ Rev 99:1714-1739.

Muller NZ, Mendelsohn R. 2012. Efficient pollution regulation: getting the prices right: corrigendum (mortality rate update). Amer Econ Rev 102:613-616.

Muller NZ, Mendelsohn R, Nordhaus W. 2011. Environmental accounting for pollution in the United States economy. Am Econ Rev 101:1649-1675. 
Pappin A, Hakami A. 2013A. Source attribution of health benefits from air pollution abatement in Canada and the United States: an adjoint sensitivity analysis. Environ Health Perspect 121:572-579.

Pappin AJ, Hakami A. 2013B. Attainment vs exposure: ozone metric responses to source-specific $\mathrm{NO}_{\mathrm{x}}$ controls using adjoint sensitivity analysis. Environ Sci Technol 47:13519-13527.

Pappin AJ, Hakami A, Blagden P, Nasari M, Szyszkowicz M, et al. 2016. Health benefits of reducing $\mathrm{NO}_{x}$ emissions in the presence of epidemiological and atmospheric nonlinearities. Environ Res Lett. 11:064015.

Pappin AJ, Mesbah SM, Hakami A, Schott S. 2015A. Diminishing returns or compounding benefits of air pollution control? The case of $\mathrm{NO}_{\mathrm{x}}$ and ozone. Environ Sci Technol 49:9548-9556.

Pappin AJ, Mesbah SM, Hakami A, Schott S. 2015B. Response to comment on "Diminishing returns or compounding benefits of air pollution control? The case of $\mathrm{NO}_{x}$ and ozone". Environ Sci Technol 50:502-503.

Peng RD, Dominici F, Pastor-Barriuso R, Zeger SL, Samet JM. 2005. Seasonal analyses of air pollution and mortality in 100 US cities. Am J Epidem 161:585-594.

Peters PA, Tjepkema M, Wilkins R, Fines P, Crouse DL, et al. 2013. Data resource profile: 1991 Canadian census cohort. Int J Epidemiol 42:1319-1326.

Pope CA III, Burnett RT, Krewski D, Jerrett M, Shi Y, et al. 2009. Cardiovascular mortality and exposure to airborne fine particulate matter and cigarette smoke: shape of the exposure-response relationship. Circulation 120:941-948. 
Pope CA III, Burnett RT, Thun MJ, Calle EE, Krewski D, et al. 2002. Lung cancer, cardiopulmonary mortality, and long-term exposure to fine particulate air pollution. JAMA 287:1132-1141.

Pope CA, Cropper M, Coggins J, Cohen A. 2015. Health benefits of air pollution abatement policy: role of the shape of the concentration-response function. J Air Waste Manag Assoc 65:516-522.

Randall A, Ives B, Eastman C. 1974. Bidding games for valuation of aesthetic environmental improvements. J Environ Econ Manage 1:132-149.

Repetto R. 1987. The policy implications of non-convex environmental damages: a smog control case study. J Environ Econ Manage 14:13-29.

Robichaud A, Ménard R. 2014. Multi-year objective analyses of warm season groundlevel ozone and $\mathrm{PM}_{2.5}$ over North America using real-time observations and the Canadian operational air quality models. Atmos Chem Phys 14:1769-1800.

Russell A, Dennis R. 2000. NARSTO critical review of photochemical models and modeling. Atmos Environ 34:2283-2324.

Samoli E, Aga E, Touloumi G, Nisiotis K, Forsberg B, et al. 2006. Short-term effects of nitrogen dioxide on mortality: an analysis within the APHEA project. Eur Respir J 27:1129-1138.

Samoli E, Peng R, Ramsay T, Pipikou M, Touloumi G, et al. 2008. Acute effects of ambient particulate matter on mortality in Europe and North America: results from the APHENA study. Environ Health Perspect 116:1480-1486. 
Sandu A, Daescu DN, Carmichael GR, Chai T. 2005. Adjoint sensitivity analysis of regional air quality models. J Comput Phys 204:222-252.

Schwartz J. 1997. Health effects of air pollution from traffic: ozone and particulate matter. In: Fletcher, T. and McMichael, A. J., Eds. Health at the Crossroads: Transport Policy and Urban Health; John Wiley \& Sons Ltd.: New York, NY.

Schwartz J, Dockery DW, Neas LM. 1996. Is daily mortality associated specifically with fine particles? J Air Waste Manage Assoc 46:927-939.

Seinfeld JH, Pandis SN. 2006. Atmospheric Chemistry and Physics: From Air Pollution to Climate Change. New York:John Wiley and Sons.

Skamarock WC, Klemp JB, Dudhia J, Gill DO, Barker DM, et al. 2005. A Description of the Advanced Research WRF Version 2. Technical Report NCAR/TN!468+STR. Boulder, CO:National Center for Atmospheric Research.

Skamarock WC, Klemp JB, Dudhia J, Gill DO, Barker DM, et al. 2008. A Description Of the Advanced Research WRF Version 3; Technical Report NCAR/TN-475+STR. Boulder, CO:National Center for Atmospheric Research.

Shin HH, Cohen A, Pope CA III, Ezzati M, Lim SS, et al. 2013. Critical Issues in Combining Disparate Sources of Information to Estimate the Global Burden of Disease Attributable to Ambient Fine Particulate Matter Exposure. Working Paper prepared for: Methods for Research Synthesis: A Cross-Disciplinary Workshop. Cambridge, MA: Harvard Center for Risk Analysis.

Starrett D. 1972. Fundamental nonconvexities in the theory of externalities. J Econ Theory 4:180-199. 
Stieb DM, Burnett RT, Smith-Doiron M, Brion O, Hyun Shin H, et al. 2008. A new multipollutant, no-threshold air quality health index based on short-term associations observed in daily time-series analyses. J Air Waste Manage Assoc $58: 435-450$.

Stieb DM, Judek S, Burnett RT. 2002. Meta-analysis of time-series studies of air pollution and mortality: effects of gases and particles and the influence of cause of death, age, and season. J Air Waste Manag Assoc 52:470-484.

Steiner AL, Tonse S, Cohen RC, Goldstein AH, Harley RA. 2006. Influence of future climate and emissions on regional air quality in California. J Geophys Res 111:D18303.

Tagaris E, Liao KJ, DeLucia AJ, Deck L, Amar P, et al. 2010. Sensitivity of air pollutioninduced premature mortality to precursor emissions under the influence of climate change. Int J Environ Res Public Health 7:2222-2237.

Thurston GD, Ito K. 2001. Epidemiological studies of acute ozone exposures and mortality. J Exp Analysis and Environ Epi 11:286-294.

Tietenberg T. 1995. Tradeable permits for pollution control when emission location matters: what have we learned? Environ Resour Econ 5:95-113.

Turner MC, Jerrett M, Pope CA III, Krewski D, Gapstur SM, Diver WR, et al. 2016. Long-term ozone exposure and mortality in a large prospective study. Am J Respir Crit Care Med 193:1134-1142. 
Turner MD, Henze DK, Hakami A, Zhao S, Resler J, et al. 2015. Differences between magnitudes and health impacts of BC emissions across the United States using 12 km scale seasonal source apportionment. Environ Sci Technol 49:4362-4371.

U.S. EPA (U.S. Environmental Protection Agency). 1999. The Benefits and Costs of the Clean Air Act, 1990-2010. EPA-410-R-99-001. Washington, DC: Office of Air and Radiation, Office of Policy Analysis and Review, U.S. Environmental Protection Agency.

U.S. EPA (U.S. Environmental Protection Agency). 2008. National Ambient Air Quality Standards for Ozone: Final Rule. Fed Reg 73:16436-16514.

U.S. EPA (U.S. Environmental Protection Agency). 2009. Integrated Science Assessment for Particulate Matter. EPA 600/R-08/139F. Research Triangle Park, NC:U.S. Environmental Protection Agency.

U.S. EPA (U.S. Environmental Protection Agency). 2010. National Ambient Air Quality Standards for Ozone: Proposed Rule. Fed Reg 2010 75:2938-3052.

U.S. EPA (U.S. Environmental Protection Agency). 2010. Guidelines for Preparing Economic Analyses. EPA 240-R-10-001. Washington, DC:U.S. Environmental Protection Agency.

U.S. EPA (U.S. Environmental Protection Agency). 2011. The Benefits and Costs of the Clean Air Act from 1990 to 2020: Final Report - Rev A. Office of Air and Radiation, U.S. Environmental Protection Agency. Available: https://www.epa.gov/sites/production/files/201507/documents/fullreport_rev_a.pdf [accessed June 12 2016]. 
U.S. EPA (U.S. Environmental Protection Agency). 2013. Integrated Science Assessment for Ozone and Related Photo-chemical Oxidants. EPA 600/R-10/076F. Research Triangle Park, NC:Environmental Protection Agency.

U.S. EPA (U.S. Environmental Protection Agency). 2015. Air Pollutant Emissions Trends Data. Available: https://www.epa.gov/air-emissions-inventories/airpollutant-emissions-trends-data [accessed 10 June 2016].

U.S. EPA (U.S. Environmental Protection Agency). 2016. Air Quality System (AQS). Available: https://www.epa.gov/aqs [accessed 24 June 2016].

West JJ, Fiore AM, Horowitz LW, Mauzerall DL. 2006. Global health benefits of mitigating ozone pollution with methane emission controls. Proc Natl Acad Sci USA 103:3988-3993.

Wild O, Prather MJ, Akimoto H. 2001. Indirect long-term global cooling from $\mathrm{NO}_{\mathrm{x}}$ emissions. Geophys Res Lett 28:1719-1722.

Wong EY, Gohlke J, Griffith WC, Farrow S, Faustman EM. 2003. Assessing the health benefits of air pollution reduction for children. Environ Health Perspect 112:226232.

WHO (World Health Organization). 2009. Global Health Risks. Available: http://www.who.int/healthinfo/global_burden_disease/global_health_risks/en/inde x.html [Accessed 10 June 2016].

Yang Y-J, Wilkinson JG, Russell AG. 1997. Fast, direct sensitivity analysis of multidimensional photochemical models. Environ Sci Technol 31:2859-2868. 
Zanobetti A, Schwartz J. 2008. Mortality displacement in the association of ozone with mortality: an analysis of 48 cities in the United States. Am J Respir Crit Care Med $177: 184-189$.

Zhang W, Capps SL, Hu Y, Nenes A, Napelenok SL, et al. 2012. Development of the high-order decoupled direct method in three dimensions for particulate matter: Enabling advanced sensitivity analysis in air quality models. Geosci Model Dev 5:355-368.

Zhao S, Pappin AJ, Mesbah SM, Zhang JYJ, MacDonald NL, et al. 2013. Adjoint estimation of ozone climate penalties. Geophys Res Lett 40:5559-5563. 


\section{Appendix A}

\section{The Chemistry of Ground-level Ozone Production}

Production of ground-level ozone $\left(\mathrm{O}_{3}\right)$ is controlled through the photochemical $\mathrm{NO}_{\mathrm{x}}$ cycle (Reactions 1-3) when $\mathrm{NO}$ and $\mathrm{NO}_{2}$ co-exist in the presence of sunlight (Seinfeld and Pandis 2006),

$$
\begin{aligned}
& \mathrm{NO}_{2}+h v \rightarrow \mathrm{NO}+\mathrm{O} \\
& \mathrm{O}+\mathrm{O}_{2}+\mathrm{M} \rightarrow \mathrm{O}_{3}+\mathrm{M} \\
& \mathrm{O}_{3}+\mathrm{NO} \rightarrow \mathrm{NO}_{2}+\mathrm{O}_{2}
\end{aligned}
$$

Reactions 1-3 account for only a fraction of observed ground-level $\mathrm{O}_{3}$. Another way by which $\mathrm{O}_{3}$ is produced is through the $\mathrm{CO}$ oxidation system (Seinfeld and Pandis 2006),

$$
\begin{aligned}
& \mathrm{CO}+\mathrm{OH} \rightarrow \mathrm{CO}_{2}+\mathrm{H} \\
& \mathrm{H}+\mathrm{O}_{2}+\mathrm{M} \rightarrow \mathrm{HO}_{2}+\mathrm{M} \\
& \mathrm{HO}_{2}+\mathrm{NO} \rightarrow \mathrm{NO}_{2}+\mathrm{OH}
\end{aligned}
$$

In the presence of atmospheric $\mathrm{NO}_{2}$, the $\mathrm{HO}_{2}$ radical produced from $\mathrm{CO}$ oxidation reacts to form $\mathrm{NO}_{2}$ and $\mathrm{OH}$ through Reaction 6 . The $\mathrm{NO}_{2}$ formed in Reaction 6 then takes part in the photochemical $\mathrm{NO}_{\mathrm{x}}$ cycle (Reactions 1-3). Without Reaction 6, the conversion of $\mathrm{NO}$ to $\mathrm{NO}_{2}$ in Reaction 3 would otherwise consume an $\mathrm{O}_{3}$ molecule. The conversion of 
$\mathrm{NO}$ to $\mathrm{NO}_{2}$ via $\mathrm{HO}_{2}$ in Reaction 6 indirectly produces an $\mathrm{O}_{3}$ molecule. Any bypassing of Reaction 3 that converts $\mathrm{NO}$ to $\mathrm{NO}_{2}$ without consuming $\mathrm{O}_{3}$ can therefore be regarded as a net production of $\mathrm{O}_{3}$ (Seinfeld and Pandis 2006).

The $\mathrm{O}_{3}$ production cycle terminates in different ways, such as with the production of nitric acid in Reaction 7; a sink for both $\mathrm{OH}$ and $\mathrm{NO}_{2}$ (Seinfeld and Pandis 2006),

$$
\mathrm{OH}+\mathrm{NO}_{2}+\mathrm{M} \rightarrow \mathrm{HNO}_{3}+\mathrm{M} \text { (7) }
$$

In addition to $\mathrm{CO}$ playing a role in $\mathrm{O}_{3}$ production, oxidation of methane and volatile organic compounds (VOCs) by $\mathrm{OH}$ occurs in the lower atmosphere and leads to further $\mathrm{O}_{3}$ production. A general form of the reaction cycle for oxidation of hydrocarbons is shown below, with RH representing a hydrocarbon chain of varying length (Seinfeld and Pandis 2006),

$$
\begin{aligned}
& \mathrm{RH}+\mathrm{OH} \rightarrow \mathrm{R}+\mathrm{H}_{2} \mathrm{O} \\
& \mathrm{R}+\mathrm{O}_{2}+\mathrm{M} \rightarrow \mathrm{RO}_{2}+\mathrm{M} \\
& \mathrm{RO}_{2}+\mathrm{NO} \rightarrow \mathrm{RO}+\mathrm{NO}_{2} \\
& \mathrm{RO}+\mathrm{O}_{2} \rightarrow \mathrm{R}^{\prime} \mathrm{CHO}+\mathrm{HO}_{2}
\end{aligned}
$$

These reactions bear many similarities to those for $\mathrm{CO}$ oxidation that take part in $\mathrm{O}_{3}$ production. Higher-carbon compounds in the form of R'CHO may continue to be oxidized after Reaction 11 and result in further $\mathrm{O}_{3}$ production. The $\mathrm{OH}$ radical is reproduced when $\mathrm{HO}_{2}$ reacts with NO through Reaction 6 (Seinfeld and Pandis 2006), 


$$
\mathrm{HO}_{2}+\mathrm{NO} \rightarrow \mathrm{NO}_{2}+\mathrm{OH}
$$

The hydrocarbon oxidation cycle terminates with the production of nitric acid as in Reaction 7, or through Reactions 13-14 (Seinfeld and Pandis 2006),

$$
\begin{aligned}
& \mathrm{HO}_{2}+\mathrm{HO}_{2} \rightarrow \mathrm{H}_{2} \mathrm{O}_{2}+\mathrm{O}_{2} \\
& \mathrm{RO}_{2}+\mathrm{HO}_{2} \rightarrow \mathrm{ROOH}+\mathrm{O}_{2}
\end{aligned}
$$

In low- $\mathrm{NO}_{\mathrm{x}}$ environments, the rate of production of $\mathrm{O}_{3}$ in the $\mathrm{CO}$ and hydrocarbon oxidation systems depends directly on the concentration of NO through Reactions 6 and 10. In this regime, $\mathrm{O}_{3}$ has a positive sensitivity to $\mathrm{NO}_{\mathrm{x}}$ emissions; any further decrease in $\mathrm{NO}_{x}$ decreases $\mathrm{O}_{3}$ production, or conversely, an increase in $\mathrm{NO}_{x}$ availability increases $\mathrm{O}_{3}$ concentrations. In such environments, $\mathrm{NO}_{\mathrm{x}}$ is the limiting reactant, and this chemical regime is often referred to as $\mathrm{NO}_{\mathrm{x}}$-limited (Seinfeld and Pandis 2006).

In high- $\mathrm{NO}_{\mathrm{x}}$ environments, $\mathrm{NO}$ is abundant for Reactions 6 and 10, but the rate of $\mathrm{O}_{3}$ production is controlled by Reactions 4 and 8 and availability of radicals that initiate these reactions. Any increase in $\mathrm{NO}_{\mathrm{x}}$ abundance in an already $\mathrm{NO}_{\mathrm{x}}$-rich environment increases removal of radicals (e.g., $\mathrm{OH}$ through Reaction 7). This removal in Reaction 7 (or similar reactions in hydrocarbon oxidation) terminates the chain reaction, reducing the rate of $\mathrm{O}_{3}$ production. Such $\mathrm{NO}_{\mathrm{x}}$-rich environments are commonly referred to as $\mathrm{NO}_{\mathrm{x}}$ inhibited, $\mathrm{NO}_{\mathrm{x}}$-saturated, radical-limited, or VOC-limited, as the presence of more $\mathrm{NO}_{\mathrm{x}}$ slows production of $\mathrm{O}_{3}$. $\mathrm{NO}_{\mathrm{x}}$-inhibited environments pose a challenge for air quality management, as controlling emissions of $\mathrm{NO}_{\mathrm{x}}$ from sources is counter-productive and leads to a rise in $\mathrm{O}_{3}$ concentrations. It is noted that the negative sensitivity of $\mathrm{O}_{3}$ to $\mathrm{NO}_{x}$ 
emissions in this chemical regime is only temporary. Gradual and continuous reductions in $\mathrm{NO}_{\mathrm{x}}$ will eventually shift the chemical regime away from $\mathrm{NO}_{\mathrm{x}}$-limited and into an environment where $\mathrm{NO}_{\mathrm{x}}$ control is favourable (Seinfeld and Pandis 2006).

The dynamics described above explain the shape of the $\mathrm{O}_{3}$ isopleth. The $\mathrm{OH}$ radical is in high demand by both $\mathrm{NO}_{\mathrm{x}}$ and VOCs in the atmosphere, as $\mathrm{OH}$ initiates the oxidation of VOCs. When VOCs are abundant relative to $\mathrm{NO}_{\mathrm{x}}$ molecules (a high ratio of VOC: $\mathrm{NO}_{x}$, such as rural areas), the predominant reaction undergone by $\mathrm{OH}$ is Reaction 8 , and $\mathrm{O}_{3}$ production is limited by $\mathrm{NO}_{x}$ availability, resulting a positive $\mathrm{O}_{3}$ sensitivity to $\mathrm{NO}_{\mathrm{x}}$ emissions. In high- $\mathrm{NO}_{\mathrm{x}}$ environments, $\mathrm{NO}_{\mathrm{x}}$ limits the availability of radicals that initiate oxidation of hydrocarbons (e.g., Reaction 8), and therefore lowering $\mathrm{NO}_{\mathrm{x}}$ emissions would enhance $\mathrm{O}_{3}$ production and result in a negative $\mathrm{O}_{3}$ sensitivity to $\mathrm{NO}_{x}$ emissions. At a certain ratio of VOC: $\mathrm{NO}_{x}$, reactions of $\mathrm{NO}$ and $\mathrm{VOCs}$ with $\mathrm{OH}$ are equally likely. This ratio depends on the mix of VOCs present and is, on average, about 5.5. $\mathrm{NO}_{\mathrm{x}}$ is therefore depleted through Reaction 7 approximately 5.5 times faster than VOCs react in Reaction 8 (Seinfeld and Pandis 2006). 


\section{Appendix B}

This appendix contains the supporting information for "Source attribution of health benefits from air pollution abatement in Canada and the United States: an adjoint sensitivity analysis"5 (Chapter 4). It is reproduced in full with permission from Environmental Health Perspectives.

\section{Supplementary Material for Chapter 4}

\section{B.1 Model Evaluation}

To evaluate the performance of the Community Multi-scale Air Quality (CMAQ) model, we compare observation-based $\mathrm{O}_{3}$ concentrations with model-predicted $\mathrm{O}_{3}$ concentrations over the July-September 2007 simulation period. Observations of $\mathrm{O}_{3}$ concentrations are taken from the U.S. EPA's Air Quality System (AQS) (U.S. EPA 2016).

Mean fractional error (MFE) is based on the absolute value of the difference between any pair of model-predicted and observation-based $\mathrm{O}_{3}$ concentrations (Russell and Dennis 2000):

$$
M F E=\frac{2}{N} \cdot \sum_{1}^{N} \frac{\left|C_{m}-C_{o}\right|}{\left(C_{m}+C_{o}\right)}
$$

\footnotetext{
${ }^{5}$ Pappin AJ, Hakami A. 2013. Source attribution of health benefits from air pollution abatement in Canada and the United States: An adjoint sensitivity analysis. Environ Health Perspect 121:572-579.
} 
where $C_{m}$ is the model-predicted ground-level $\mathrm{O}_{3}$ concentration (layer 1) in a particular location and time, $C_{o}$ is the $\mathrm{O}_{3}$ concentration observed at a monitor located in the same grid cell and at the same time, and $\mathrm{N}$ is the number of observations across all locations and times. If more than one monitor exists at a grid cell, an average of their measurements is used.

Mean fractional bias (MFB) quantifies the directional (i.e., positive or negative) tendency of CMAQ to over or under-estimate $\mathrm{O}_{3}$ concentrations compared to observations (Russell and Dennis 2000):

$$
M F B=\frac{2}{N} \cdot \sum_{1}^{N} \frac{\left(C_{m}-C_{o}\right)}{\left(C_{m}+C_{o}\right)}
$$

\section{B.2 Supporting Figures}

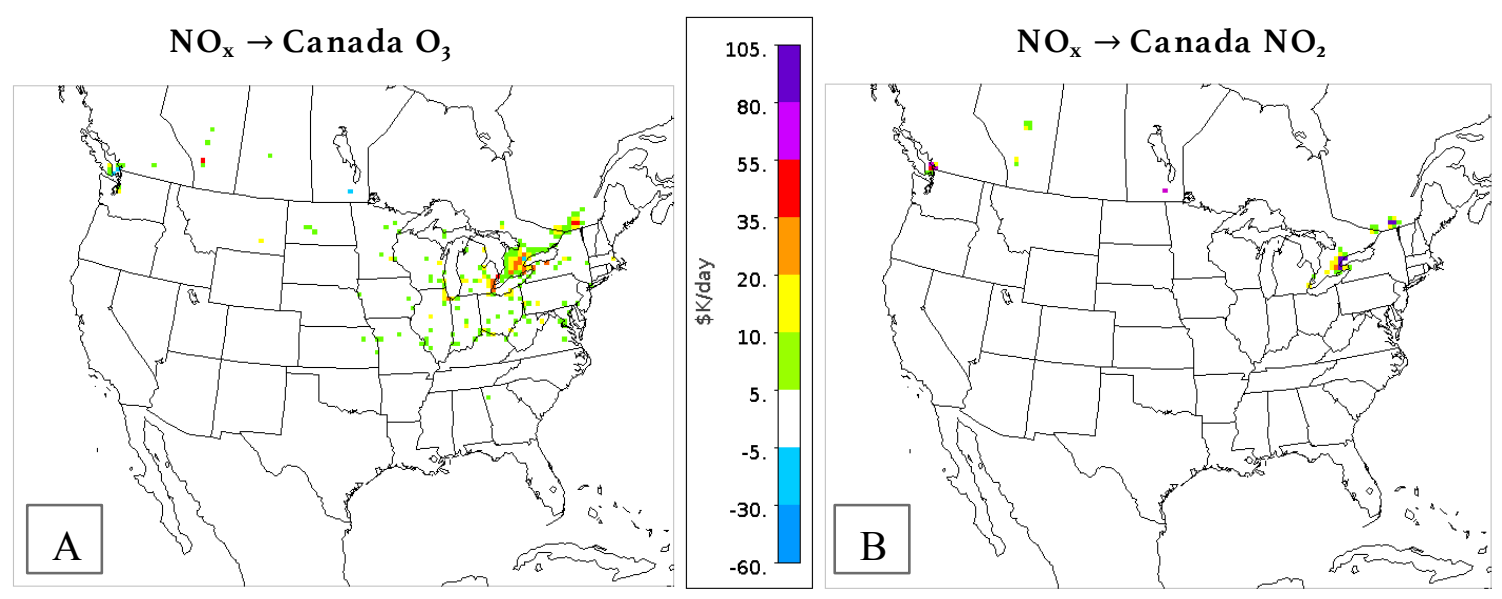

Figure B1. Average daily influences on Canada short-term mortality related to (A) $\mathrm{O}_{3}$ and (B) $\mathrm{NO}_{2}$ from a $10 \%$ reduction in anthropogenic emissions of $\mathrm{NO}_{\mathrm{x}}$. Health benefit influences are estimated based on (A) daily 1-hr maximum $\mathrm{O}_{3}$ concentrations and (B) 24-hr average $\mathrm{NO}_{2}$ concentrations separately. 


$$
\mathrm{NO}_{\mathrm{x}} \rightarrow \text { Canada } \mathrm{O}_{3}+\mathrm{NO}_{2}
$$
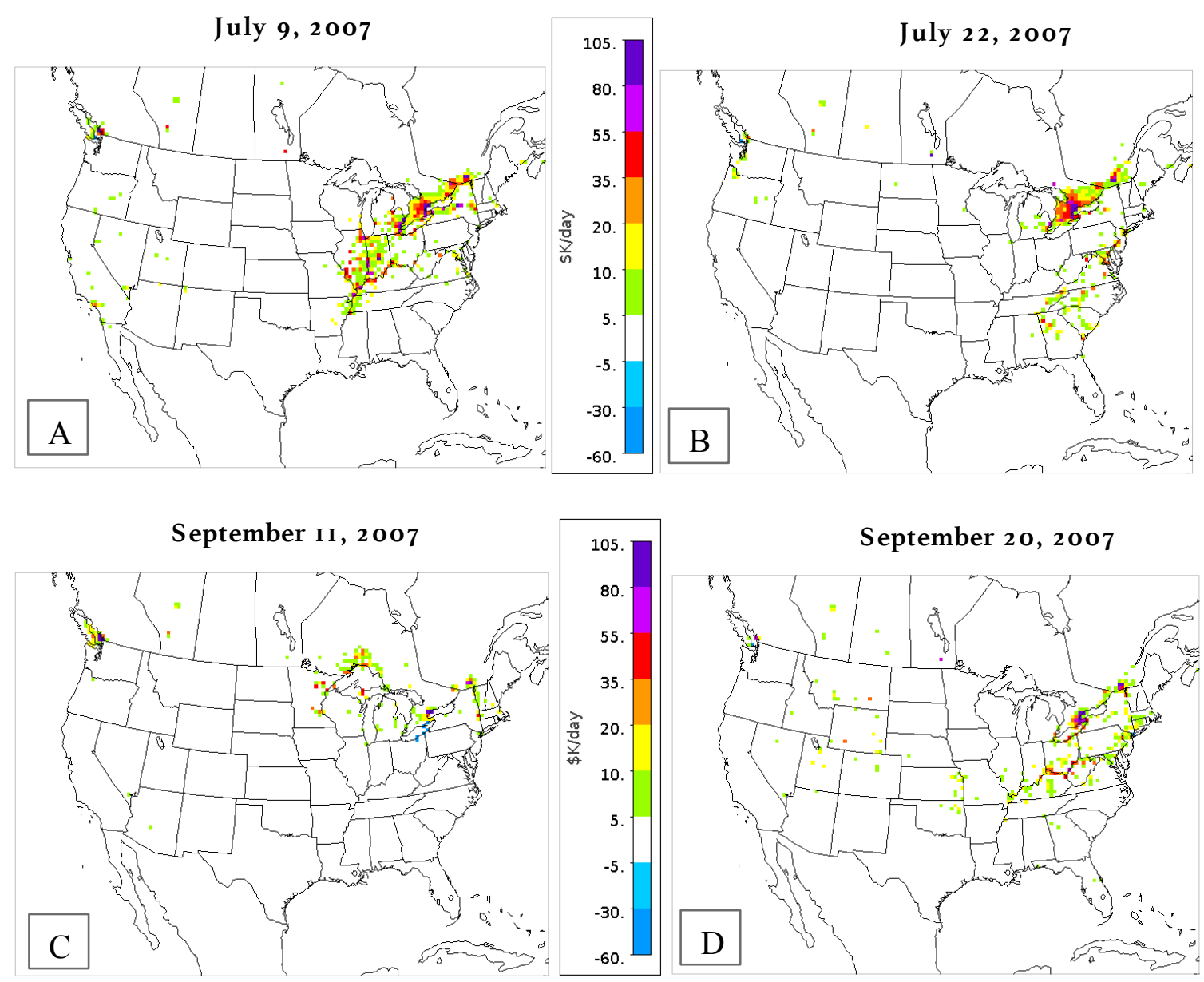

Figure B2. Daily variability of influences from a $10 \%$ reduction in anthropogenic emissions of $\mathrm{NO}_{\mathrm{x}}$ originating from individual source locations on Canada short-term mortality related to both $\mathrm{O}_{3}$ and $\mathrm{NO}_{2}$ exposure. Snapshots are shown for (A) July 9, (B) July 22, (C) September 11 and (D) September 20 during 2007. 

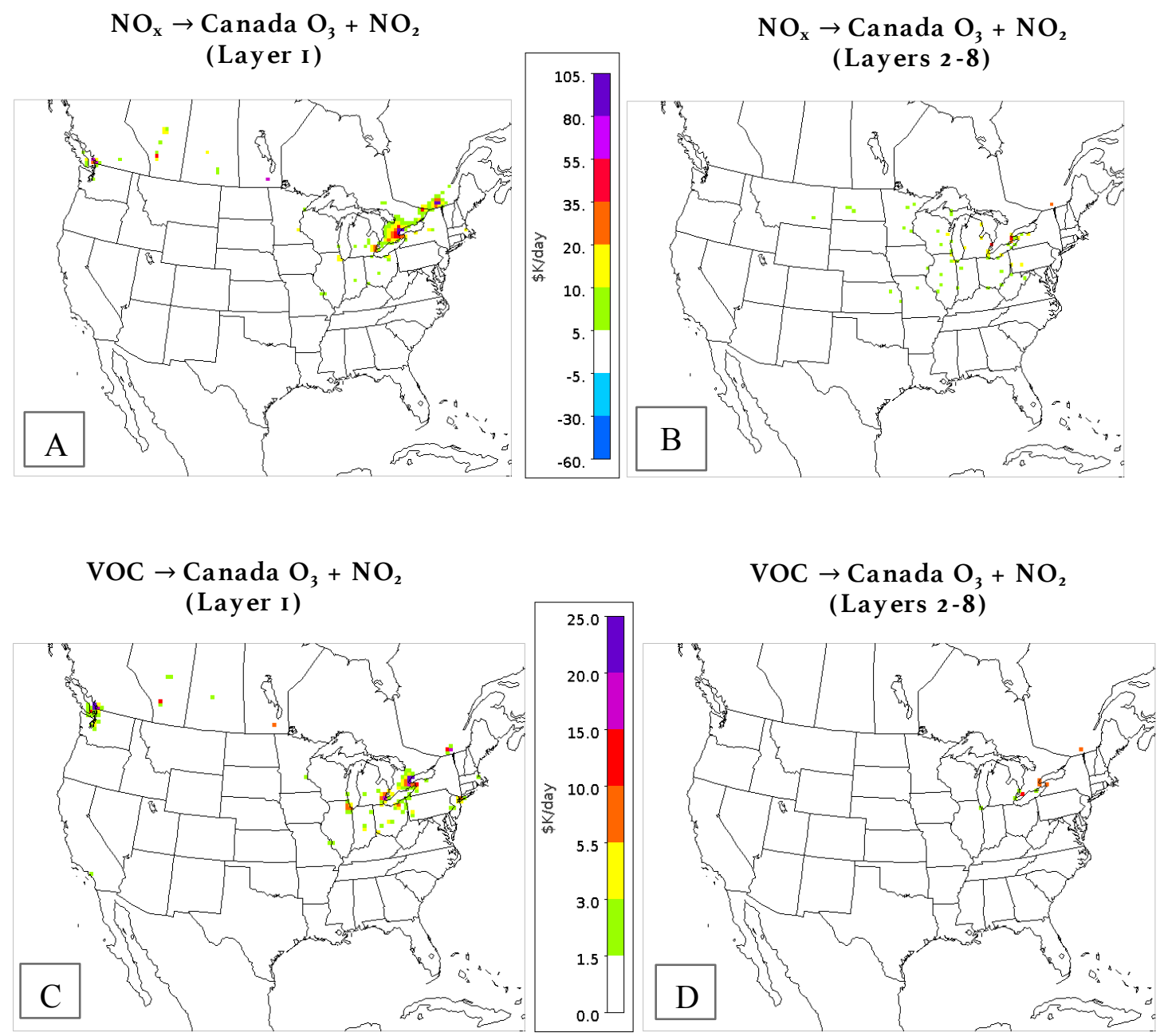

Figure B3. Average daily influences of a $10 \%$ reduction in anthropogenic emissions of $\mathrm{NO}_{\mathrm{x}}(\mathrm{A}, \mathrm{B})$ and VOCs (C,D) on Canada short-term mortality from surface-level emissions (left) and elevated sources (right). 


\section{Appendix C}

This appendix contains the supporting information for "Attainment vs Exposure: Ozone Metric Responses to Source-specific $\mathrm{NO}_{\mathrm{x}}$ Controls Using Adjoint Sensitivity Analysis" (Chapter 5). It is reprinted (adapted) with permission from Pappin AJ, Hakami A. 2013. Attainment vs exposure: Ozone metric responses to source-specific $\mathrm{NO}_{\mathrm{x}}$ controls using adjoint sensitivity analysis. Environ Sci Technol 47:13519-13527. Copyright 2013 American Chemical Society.

\section{Supplementary Material for Chapter 5}

\section{C.1 Formulation of Attainment-Based Adjoint Forcing Terms}

Adjoint forcing terms are calculated by differentiating the attainment-based adjoint cost function, $J_{a}$, with respect to daily maximum 8-hr average (DM8A) concentrations on each day, and are unique to each location. Adjoint forcing terms thus depend on the DM8A ozone concentration distribution, as formulated below for each location (each $k$ in Equation $\mathrm{C} 1$ ),

$$
\varphi_{i}=\partial J_{a} /_{\partial x_{i}}=\frac{p_{T}\left(x_{i} p_{i}{ }^{\prime}+x_{i}{ }^{\prime} p_{i}\right)-p_{i}{ }^{\prime} \sum_{i}^{n} x_{i} p_{i}}{p_{T}{ }^{2}}
$$

where $x_{i}$ is the simulated DM8A ozone concentration (ppb) for day $i$ for the 8 -hr interval from $t_{0} \rightarrow t_{f}, p_{i}$ is the probability that $x_{i}$ is among the four highest ozone concentrations modeled in the season, and $p_{T}$ is the summation of all $p_{i}$ over $n$ simulation days. $p_{i}{ }^{\prime}$ is 
the derivative of $p_{i}$ with respect to $x_{i}$, and $x_{i}{ }^{\prime}$ is the derivative of the DM8A concentration with respect to the ozone concentration at the model time step in which the adjoint forcing term is injected (similarly to how emission terms are injected into forward chemical transport models). The derivative $p_{i}^{\prime}$ is formulated as

$$
p_{i}{ }^{\prime}=\sum_{j=0}^{3}\left[\left(\begin{array}{l}
n \\
j
\end{array}\right) p_{x}{ }^{j}\left(1-p_{x}\right)^{n-j} p_{x}{ }^{\prime} \times \frac{j-n p_{x}}{p_{x}\left(1-p_{x}\right)}\right]
$$

where $p_{x}$ is the probability that a randomly selected ozone concentration exceeds that day's simulated concentration, which can be related to the cumulative distribution function $(c d f)$,

$$
p_{x}=P\left(X>x_{i}\right)=1-c d f
$$

thus, the derivative $p_{x}{ }^{\prime}$ can be related to the probability density function $(p d f)$ for $x_{i}$, as shown below,

$$
p_{x}{ }^{\prime}=-p d f=-\frac{1}{\sigma \sqrt{2 \pi}} \times \exp \left(\frac{-\left(x_{i}-\mu\right)^{2}}{2 \sigma^{2}}\right)
$$

where $\mu$ and $\sigma$ are the mean and standard deviation of the simulated DM8A ozone concentration distribution, respectively. 


\section{C.2 Supporting Figures}

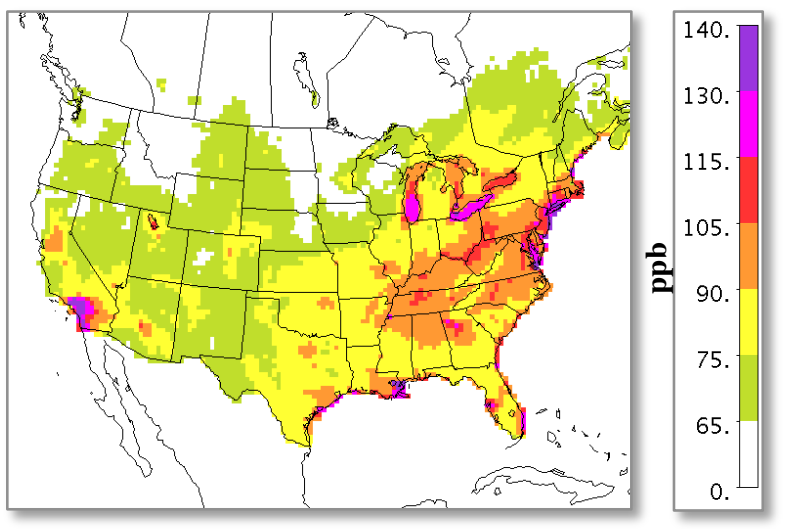

Figure C1. Local probabilistic design values (PDVs) exceeding 65 ppb across receptor locations in Canada and the U.S. PDVs are calculated using the modeled DM8A ozone concentrations from May 1 - September 30, 2007. Spatial integration across all receptors yields the attainment-based adjoint cost function, $J_{a}$.

relative contribution to reduction in PDVs

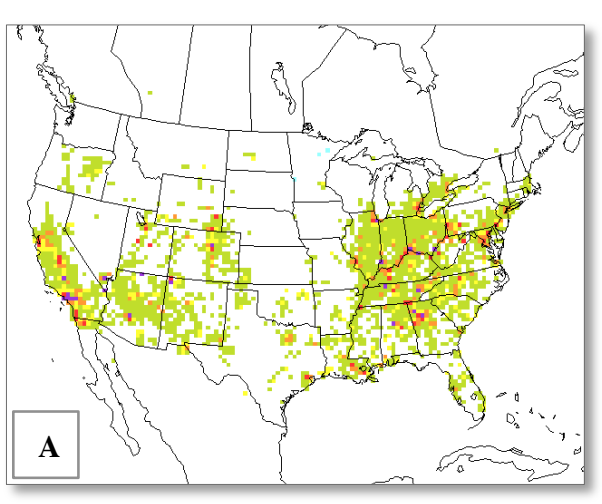

relative contribution to reduction in mortality

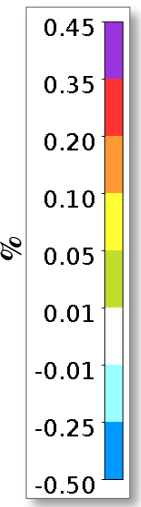

Figure C2. Normalized influences of a $10 \%$ reduction in anthropogenic $\mathrm{NO}_{\mathrm{x}}$ from each source location on the (A) attainment-based metric and (B) the exposure-based metric. The value in each grid cell represents the percentage contribution of source reductions in that grid cell to the overall influence if anthropogenic $\mathrm{NO}_{\mathrm{x}}$ emissions were reduced everywhere in the continent. Note that we do not attempt to account for all source influences (particularly those from sources outside of the 
domain boundaries); we simply scale our results for comparison of the relative importance of emission controls among the two metrics. Influences are integrated over May 1 - September 30, 2007.

reduction in mortality, 1-hr (for $10 \% \downarrow \mathrm{NO}_{\mathrm{x}}$ )

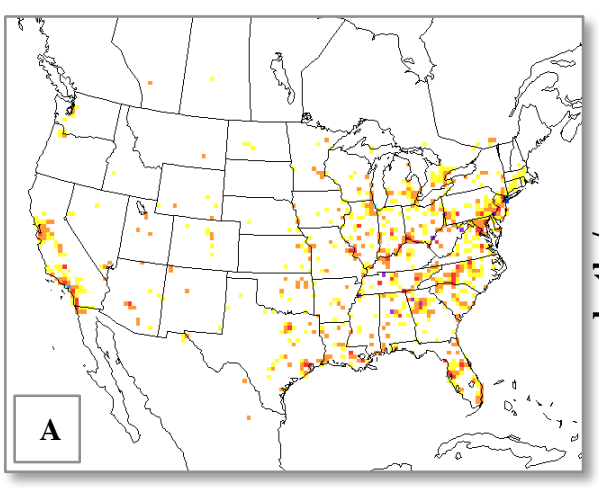

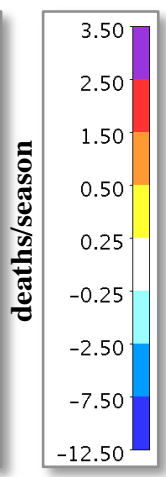

reduction in mortality, 8 -hr (for $\left.10 \% \downarrow \mathrm{NO}_{\mathrm{x}}\right)$

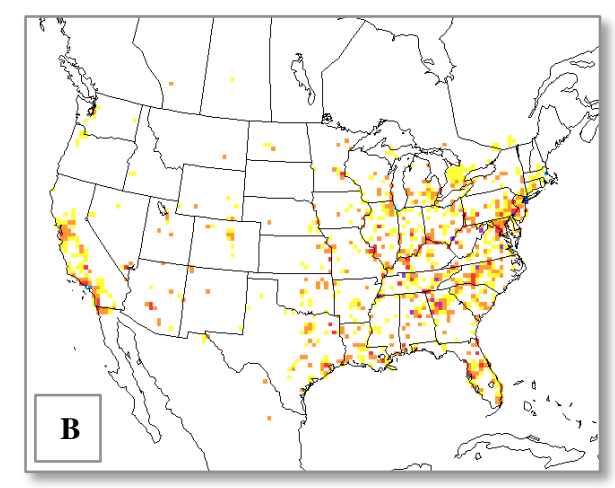

reduction in mortality, 24-hr (for $10 \% \downarrow \mathrm{NO}_{\mathrm{x}}$ )
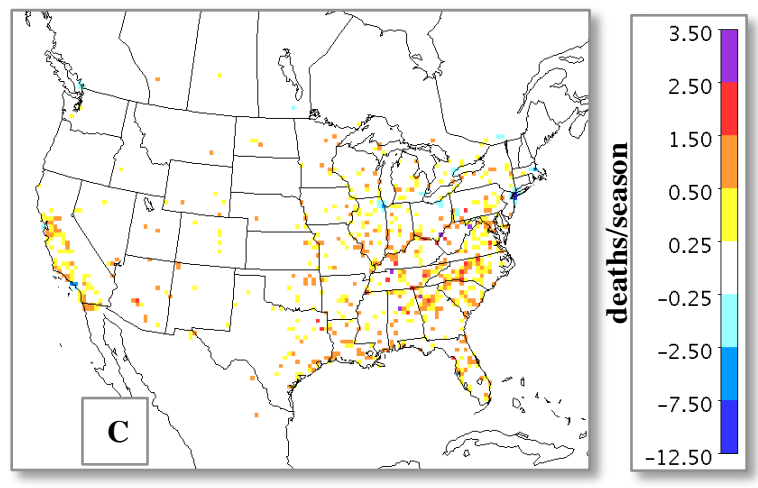

Figure C3. Influences of a $10 \%$ reduction in anthropogenic $\mathrm{NO}_{\mathrm{x}}$ from each source location on exposure-based metrics based on averaging periods of 1 hour (A), 8 hours (B), and 24 hours (C). Influences are integrated over May 1 - September 30, 2007. 


\section{Appendix D}

This appendix contains a reproduction of the article entitled 'Response to Comment on "Diminishing Returns or Compounding Benefits of Air Pollution Control? The Case of $\mathrm{NO}_{\mathrm{x}}$ and Ozone"'. This article is a response to a comment made on the original research article (Chapter 6; Pappin et al. 2015A). The author response is reprinted (adapted) with permission from Pappin AJ, Mesbah SM, Hakami A, Schott S. 2015. Response to comment on "Diminishing returns or compounding benefits of air pollution control? The case of $\mathrm{NO}_{\mathrm{x}}$ and ozone”. Environ Sci Technol 50:502-503. Copyright 2015 American Chemical Society. The comment on which this response is based is not reproduced here due to copyright. The full citation for the comment is listed below,

Mendelsohn R, Muller NZ. 2015. Comment on "Diminishing returns or compounding benefits of air pollution control? The case of $\mathrm{NO}_{\mathrm{x}}$ and ozone". Environ Sci Technol 50:500-501.

\section{Response to Comment on Chapter 6}

Mendelsohn and Muller (2015) raise concerns about our paper (Pappin et al. 2015A) on the non-convex response of ozone health damages to $\mathrm{NO}_{\mathrm{x}}$ emissions abatement. We thank the authors for their comments, but believe that they stem from various misconceptions. 
First, Mendelsohn and Muller (2015) mention that our findings are not supported by previous studies of ozone nonlinearity or its isopleth. Using a depiction of the ozone isopleth (Figure 3; Mendelsohn and Muller 2015), they argue that MBs do not increase with $\mathrm{NO}_{\mathrm{x}}$ abatement. However, an important feature of this particular choice of isopleth, and one that the authors may have overlooked, is the logarithmic scale for both $\mathrm{NO}_{\mathrm{x}}$ and VOCs. The nonlinear scale of Figure 3 skews the shape and spacing of the contour lines, but examination of other ozone isopleths clearly supports our finding of increasing MBs with $\mathrm{NO}_{\mathrm{x}}$ abatement. While our study was the first to put forth nonconvexity as the general rule for ozone and $\mathrm{NO}_{\mathrm{x}}$, we discuss in detail previous cases in the literature indicating prevalent nonconvexity for both ozone and PM (see Fann et al. 2012A; Hakami et al. 2003; Hakami et al. 2004; Zhang et al. 2012).

Second, Mendelsohn and Muller (2015) take exception to our claim that where $\mathrm{MB}>\mathrm{MC}$, rising MBs provide "economic incentive for higher levels of abatement than were previously advisable". They argue that these findings do not change the existing economic advice, and that "[if] previous advice is to equate MC to MB, Figure 5 supports that advice." However, Mendelsohn and Muller (2015) misunderstand the main point we convey in Figure 5 of our paper. We never claim that the economic advice changes (i.e., equating $\mathrm{MC}$ to $\mathrm{MB}$ ); only that this same economic advice leads to higher levels of abatement in the presence of rapidly rising MBs.

Third, Mendelsohn and Muller (2015) point out that the case of a rising positive MB function is only one possible example (Figures 1-2 in their comment), and argue that in cases with negative MBs, "current regulations [may] encourage too much abatement". While the prevalent behavior across the domain is that of positive MBs (Figure 1 in 
Pappin et al. 2015A), cases with negative MB represent two of the most populous cities in the U.S. Figure 1 in Mendelsohn and Muller (2015) shows that, as expected, major point sources in Los Angeles are not important players in determining its chemical regime. This case is irrelevant, as point sources do not significantly impact the efficiency of control strategies in this city. Figure 2 in Mendelsohn and Muller (2015) offers a more realistic case for mobile sources in the cities of Los Angeles and New York. We agree that the second-order condition fails at the intersection point in this figure, but the main reason for this failure is the nonconvexity that we find at all levels of MB. Furthermore, Mendelsohn and Muller (2015) choose an example for which MC $<$ MB at 100\% abatement. We point out that achieving zero emissions in cities like Los Angeles or New York is nearly impossible for the foreseeable future, and therefore, $\mathrm{MC}$ is likely to be infinitely large at $100 \%$ abatement. As such, a second intersection point would exist at very high levels of abatement for which the second order condition is satisfied. Existence of this second intersection point, together with the very rapid rise in MBs (driven by large populations), indicates that even in the case of negative MBs posed by Mendelsohn and Muller (2015), nonconvexity may justify a more aggressive control policy.

One should also note that $\mathrm{NO}_{\mathrm{x}}$ emissions in these large cities are decreasing for a spectrum of reasons that go beyond economic analysis, such as attainment efforts for PM and downwind ozone, technology based controls (e.g., vehicle standards), and climate objectives and/or policies. Through such emission reductions, these cities are on the path of change from a disbenefit-inducing $\mathrm{NO}_{\mathrm{x}}$-inhibited chemical regime to a $\mathrm{NO}_{\mathrm{x}}$-limited one that entails large benefits associated with $\mathrm{NO}_{\mathrm{x}}$ abatement. More aggressive $\mathrm{NO}_{\mathrm{x}}$ control in these cities would allow for future abatement measures that are aligned with, 
rather than contradict, the larger landscape of air quality and climate polices.

Fourth, Mendelsohn and Muller (2015) express concern about changing emissions of VOCs when evaluating $\mathrm{NO}_{\mathrm{x}} \mathrm{MBs}$. We maintain that changing emissions of all species provides a more realistic representation of the atmosphere along the abatement trajectory. We note, however, that with zero or smaller reductions in VOC emissions, the pace of increase in $\mathrm{NO}_{\mathrm{x}} \mathrm{MBs}$ is even faster than those we report previously (Pappin et al. 2015A). For example, mobile $\mathrm{NO}_{\mathrm{x}} \mathrm{MBs}$ in Los Angeles are $\$ 77,000 /$ ton if only abating $\mathrm{NO}_{\mathrm{x}}$ from motor vehicles by $80 \%$, as compared to $\$ 49,000 /$ ton that we report from abating all species (Figure 2; Pappin et al. 2015A). Policies that target more aggressive VOC control may seem an appropriate option for large cities; however, their efficacy is limited by vehicle technology standards and the VOC concentration floor imposed by biogenic emissions.

Fifth, Mendelsohn and Muller (2015) argue that MBs should be location-specific. We note that our use of adjoint modeling does provide location-specific (and species- or time-specific) MBs. We apply across-the-board reductions in emissions to evaluate how location-specific MBs change in the larger scheme of changes in the atmosphere. Sourcespecific estimation of these impacts (accounting for all possible combinations of derivatives and cross-derivatives) poses an insurmountable computational cost. Using simplified models such as the Air Pollution Emission Experiments and Policy analysis (APEEP; Muller and Mendelsohn 2009) may seem tempting, but these models do not provide adequate representation of nonlinear atmospheric processes that give rise to rapidly changing MBs and have underestimated MBs compared to models that contain more sophisticated representation of atmospheric processes (Fann et al. 2012A; 
Mauzerall et al. 2005; Pappin and Hakami 2013A). Even for models such as APEEP, calculating all possible source-specific MB curves remains a daunting task. For example, characterizing all MB curves in a model configuration with 100,000 sources (a modest estimate) would require $\left[1+100,000+(100,000)^{2} / 2\right]$ simulations. Most importantly, and apart from the computational challenges involved, we believe that our representation provides a more realistic view of a changing atmosphere, as "[changes] in sectoral emissions $[\ldots]$ seldom happen in isolation and commonly materialize within a broader, nationwide context" (Pappin et al. 2015). 